\title{
FREE ANALYSIS QUESTIONS II: THE GRASSMANNIAN COMPLETION AND THE SERIES EXPANSIONS AT THE ORIGIN
}

\author{
DAN-VIRGIL VOICULESCU
}

\begin{abstract}
The fully matricial generalization in part I, of the difference quotient derivation on holomorphic functions, in which $\mathbb{C}$ is replaced by a Banach algebra $B$, is extended from the affine case to a Grassmannian completion. The infinitesimal bialgebra duality, the duality transform generalizing the Stieltjes transform and the spectral theory with non-commuting scalars all extend to this completion. The series expansions of fully matricial analytic functions are characterized, providing a new way to generate fully matricial functions.
\end{abstract}

\section{INTRODUCTION}

In our first paper in this series [16], we produced a fully matricial object which generalizes from the complex numbers $\mathbb{C}$ to a unital Banach algebra $B$ the structure on $\mathbb{C}$ in which on each open subset of $\mathbb{C}$ we are given the bialgebra of scalar analytic functions with the comultiplication defined by the difference quotient derivation. One main theme of the present paper can be described as the extension of the construction from the affine line to the Riemann sphere. This means that with $\mathbb{C}$ replaced by $B$, we will exhibit a fully matricial object generalizing the Riemann sphere and the bialgebras of analytic functions on open sets with respect to the difference quotient. This will be achieved by tying together certain Grassmannians for the matrices $\mathfrak{M}_{n}(B)$.

Among the other main questions we study are the series expansions at the origin for fully matricial functions. Using our results on the series expansions we are able to clarify the structure of fully matricial analytic functions. For the bounded functions in the matricial analogue of the disk we show that the non-commutative $H^{\infty}$-spaces which arise are

2000 Mathematics Subject Classification. Primary 16W30, 46L54; Secondary 47A56, 47C15, 46G20.

Subjects. OA, QA, CV.

Key words and phrases. Grassmannian completion, bialgebra duality, free difference quotient, fully matricial analytic function, generalized spectral theory, large $N$ limit. 
connected to certain non-self-adjoint algebras in full free product $C^{*}$ algebras $\left(\mathfrak{M}_{k} *_{\mathbb{C}} C(\mathbb{T})\right.$ in case $B=\mathfrak{M}_{k}$ and the full free group $C^{*}$-algebra in case $\left.B=\mathbb{C}^{n}\right)$. A further feature of these matricial holomorphic functions is that they naturally have a large $N$ limit which lives in certain non-self-adjoint subalgebras of free group $I I_{1}$-factors.

There are several reasons for passing to the Grassmannian completion. One reason is the duality transform for the coalgebra of the free difference quotient ([16] see also [15]). We used only a part of the corepresentations (i.e., group-like elements) which arise from generalized matricial resolvents. Clearly, this means the matricial analytic functions of the duality transform have analytic extensions to a larger natural domain of definition and we should try to find these extensions.

Another motivation is provided by spectral theory. Duality for the free difference quotient can be viewed as an extension of the spectral analysis of an operator from the case of complex scalars $\mathbb{C}$ to the case of an algebra $B$ of scalars which may not commute with the operator. To handle the behavior at infinity of the generalized resolvents it is natural to use the Grassmannian. This is also convenient for dealing with unbounded self-adjoint operators. Note that similar motives underlie the use of Grassmannians in operator theory problems of electrical engineering (see [6]).

The point at infinity of the Grassmannian completion is also well suited for dealing with the fully matricial version of the operator-valued $R$-transform ([13]). The result on the series expansion when applied to this fully matricial $R$-transform yields a noncommutative series $R$ transform, which in view of formal analogies coincides with the unsymmetrized $R$-transform of [5] (where also the relation to free comulants [10] is discussed).

A central result of our paper, the proof of which is scattered through several sections, can be stated roughly as follows.

Fact. If $B$ is a unital complex Banach algebra and $\Omega$ a fully matricial $B$-set of the Grassmannian, then the fully matricial scalar analytic functions $\mathcal{A}(\Omega)$ form a "topological" infinitesimal bialgebra ([8], [1]) (or GDQ ring in the terminology which we used in [15], [16]) $(\mathcal{A}(\Omega), \mu, \widetilde{\partial})$ with multiplication $\mu: \mathcal{A}(\Omega ; \Omega) \rightarrow \mathcal{A}(\Omega)$ and comultiplication-derivation $\widetilde{\partial}: \mathcal{A}(\Omega) \rightarrow \mathcal{A}(\Omega ; \Omega)$ and with coassociativity requirements stated in terms of $\mathcal{A}(\Omega ; \Omega ; \Omega)$. Moreover, there is a coderivation $\Lambda: \mathcal{A}(\Omega) \rightarrow$ $\mathcal{A}(\Omega)$ with respect to $\widetilde{\partial}$ such that $\Lambda-$ id is a derivation of $\mathcal{A}(\Omega)$. If $B$ is a Banach algebra with involution and $\Omega=\Omega^{*}$, then there is an 
involution on $\mathcal{A}(\Omega)$, compatible with $(\mathcal{A}(\Omega), \mu, \widetilde{\partial}, \Lambda)$ (that is, satisfying the "topological" version of Definition 5.1 of [15]).

For the precise definitions of certain terms in the previous statement, it is necessary to read the paper beyond the introduction. Note also, that the fully matricial affine space, to which the considerations in $[16]$ were restricted, is an open subset of the Grassmannian. In case $B=\mathbb{C}$, $\widetilde{\partial}$ is the difference quotient $\left(z_{1}-z_{2}\right)^{-1}\left(f\left(z_{1}\right)-f\left(z_{2}\right)\right)$ acting on analytic functions on an open subset of the Riemann sphere. The difference quotient $\widetilde{\partial}$ is not invariant under automorphisms of $\mathbb{P}^{1}(\mathbb{C})$ and there is a certain vanishing of $\widetilde{\partial} f$ at infinity.

Another main result is the extension of the duality transform to the Grassmannian context. Part of this can also be seen as the extension of the related non-commutative spectral theory, with particular emphasis on the cases of unitary and self-adjoint operators. While the properties of the duality transform relative to an operator, or graph of operator, and an "algebra of scalars" depend on whether certain technical conditions are satisfied, we show in the unitary and self-adjoint cases that one can always pass to the universal such operators which satisfy these requirements and use the corresponding universal duality transforms.

We have also included in this second paper new results in the affine context, like the sub-bialgebra of polynomial functions on the fully matricial affine space. This clarifies the action of the generalized difference quotient and underlies the series expansions at the origin and the beginnings of a corresponding study of functions in the analogue of the disk.

It is not clear whether the non-commutative $H^{\infty}$-spaces which we encounter here can benefit from the recent progress on the non-commutative $H^{\infty}$-spaces arising from generalizations of the shift operator and of Fock spaces (see for instance [9] and the references therein).

There is a certain peculiarity of the Grassmannian completion that should be pointed out. In the affine framework of the preceding paper ([16]), for most purposes it was sufficient to assume that $B$ is just a Banach space with a distinguished unit vector and sometimes to add to this a conjugate-linear involution. The Grassmannian completion uses the fact that $B$ is an algebra, that is that there is a multiplication operation. It is conceivable that while the multiplication may be necessary, its associativity could be relaxed.

The paper has seventeen sections, including the introduction and there are also two Appendices. Some preliminaries from [16] are briefly recalled in section 2 , but we could only cover a part of the instances 
where it is necessary to go back to [16]. Section 3 introduces the fully matricial Grassmannian and its fully matricial sets and fully matricial functions. Grassmannian resolvents and resolvent sets are the subject of section 4 . The long section 5 is devoted to the definition of the generalization of the difference quotient derivation to the fully matricial $B$-Grassmannian and to checking its basic properties. Section 6 is also one of the longer sections of the paper and deals with the Grassmannian resolvent equation and duality transform. The important algebras $\mathcal{L} \mathcal{R}(\pi ; B)$ of coefficients of Grassmannian resolvents are also introduced in section 6 . The polynomial bialgebra on the fully matricial affine space and some basic remarks about the fully matricial affine space are the subject of section 7 . We then return to the Grassmannian in section 8 and we define the coderivation $\Lambda$ and check its properties with respect to the bialgebra structure and duality transform. In section 9 we turn to the involution on the Grassmannian and to its properties with respect to the bialgebra structure and duality transform. (This is an instance when the arguments in the affine case are substantially shorter.) We take up dual positivity and duality transforms of positive functionals in the Grassmannian context in section 10. The generalizations to our context of certain spectrally important sets such as half-planes and the unit ball leads to stably matricial sets, which are more general than fully matricial sets, and to which we devote section 11. We also introduce in section 11 the mixed unit balls and the mixed half-planes, which are always present in the fully matricial resolvents of unitary and respectively of hermitian elements in addition to the usual unit ball and upper and lower half-planes. We conclude section 11 with results about the use of stably matricial sets for obtaining fully matricial inverse function results. Section 12 collects some further remarks on the duality transforms for unitary and hermitian elements and introduces the universal unitary and hermitian Grassmmannian elements. We show that certain technical assumptions for the duality transform appearing in previous sections hold in the universal case. Section 13 establishes the form of the series expansions at the origin for fully matricial analytic functions and their behavior under composition of such functions. We should mention that using automorphisms of the fully matricial Grassmannian this also can be used for instance for the series expansions for the point at infinity or for " $B$-points of the affine space" that is for fully matricial sets $\left(\left\{b \otimes I_{n}\right\}\right)_{n \in \mathbb{N}}$. In section 14 we find the analogue in case $B=\mathfrak{M}_{k}$ for the formulae connecting Fourier coefficients of boundary values on the circle to coefficients of the Taylor series at the origin. The analogue of the integrations on the circle is here the limit of the integrations over the unitary groups $U\left(\mathfrak{M}_{N}\left(\mathfrak{M}_{k}\right)\right)$. 
We also show that the analogue of the $H^{\infty}$-algebra in this case is related to a subalgebra of the full free product $\mathfrak{M}_{k} *_{\mathbb{C}} C(\mathbb{T})$. In section 15 we use the results of the preceding section to show in the case $B=\mathfrak{M}_{k}$ the existence of a large $N$ limit of the functions which are in the analogue of $H^{\infty}$ of the unit disk. The large $N$ limit is in a non-self-adjoint subalgebra of a $I I_{1}$-factor. Section 16 deals with the analogues in case $B=\mathbb{C}^{k}$ of the results of sections 14 and 15 . Here the related $C^{*}$ algebra is the full $C^{*}$-algebra of a free group $C^{*}\left(F_{k}\right)$ and the related von Neumann algebra is the free group factor $L\left(F_{k}\right)$. In section 17 , we give a construction of pathological fully matricial analytic functions in the absence of boundedness conditions.

For a better perspective we give in Appendix I the classical case of the difference quotient bialgebra duality on the Riemann sphere. Checking the duality relations amounts to familiar applications of the Cauchy integral formula. In a second appendix (Appendix II) we briefly explain how the $B$-valued $R$-transform ([13]) gives rise naturally to a fully matricial $R$-transform and this in turn to a noncommutative series $R$-transform which is precisely the unsymmetrized $R$-transform of [5]. In this way all $B$-moments, not only the symmetric ones, are encoded in a generalization of the analytic $R$-transform.

\section{Preliminaries}

2.1. Let $E$ be a complex Banach algebra with 1 and $1 \in B \subset E$ a Banach subalgebra and let $X \in E$. The Banach algebra $B$ will play the role of "scalars" in the spectral analysis of $X$. Note that $X$ and $B$ do not commute in general. Also for the considerations in this section we could have replaced $B$ by a Banach subspace $\mathcal{U}, 1 \in \mathcal{U} \subset E$. Later on we will be especially interested in the case where $E=\mathcal{B}(\mathcal{H})$ is the algebra of bounded operators on a Hilbert space $\mathcal{H}, B$ a $C^{*}$-subalgebra of $E$ and $X$ is a self-adjoint operator. The material in this section (with slight adaptations) is from [16].

2.2. The $n \times n$ matrices with entries in $B$, denoted $\mathfrak{M}_{n}(B)$ can be identified with $\mathfrak{M}_{n} \otimes B$, where $\mathfrak{M}_{n}$ is short for $\mathfrak{M}_{n}(\mathbb{C})$ and the tensor product is over $\mathbb{C}$. Then $I_{n} \otimes X \in \mathfrak{M}_{n} \otimes E \simeq \mathfrak{M}_{n}(E)$ is the diagonal matrix with diagonal entries equal $X$, which we will also denote sometimes by $\underbrace{X \oplus \cdots \oplus X}_{n}$.

For each $n \geq 1$, the $n$-th $B$-resolvent set of $X$, is the set

$$
\rho_{n}(X ; B)=\left\{\beta \in \mathfrak{M}_{n}(B) \mid I_{n} \otimes X-\beta \text { invertible }\right\} .
$$


The sequence of sets $\rho(X ; B)=\left(\rho_{n}(X ; B)\right)_{n \in \mathbb{N}}$ will be called the full $B$-resolvent set of $X$.

2.3. The full $B$-resolvent set of $X$ is an example of a fully matricial $B$-set. More generally, if $\mathcal{U}$ is a Banach space over $\mathbb{C}$, a fully matricial $\mathcal{U}$-set $\Omega$ is a sequence $\Omega=\left(\Omega_{n}\right)_{n \in \mathbb{N}}, \Omega_{n} \subset \mathfrak{M}_{n}(\mathcal{U})$ so that $\Omega_{m+n} \cap$ $\left(\mathfrak{M}_{m}(\mathcal{U}) \oplus \mathfrak{M}_{n}(\mathcal{U})\right)=\Omega_{m} \oplus \Omega_{n}$ and

$$
\left(\operatorname{Ad} S \otimes I_{\mathcal{U}}\right)\left(\Omega_{n}\right)=\Omega_{n}
$$

if $S \in G L(n ; \mathbb{C}) \subset \mathfrak{M}_{n}(\mathbb{C})$. Here Ad $S$ denotes the inner automorphism $T \rightsquigarrow S T S^{-1}$ of $\mathfrak{M}_{n}$ and $I_{\mathcal{U}}$ is the identity operator on $\mathcal{U}$.

2.4. On each set $\rho_{n}(X ; B)$ there is an analytic function

$$
R_{n}(X ; B): \rho_{n}(X ; B) \rightarrow \mathfrak{M}_{n}(E)
$$

defined by

$$
R_{n}(X ; B)(\beta)=\left(I_{n} \otimes X-\beta\right)^{-1} .
$$

The sequence $R(X ; B)=\left(R_{n}(X ; B)\right)_{n \in \mathbb{N}}$ is called the full $B$-resolvent of $X$.

2.5. The full $B$-resolvent of $X$ is an example of a fully matricial $E$ valued function. More generally if $\Omega=\left(\Omega_{n}\right)_{n \in \mathbb{N}}$ is a fully matricial $\mathcal{U}$-set and $\mathcal{V}$ is another Banach space, then a fully matricial $\mathcal{V}$-valued function on $\Omega$ is a sequence $f=\left(f_{m}\right)_{n \in \mathbb{N}}$ where

$$
f_{n}: \Omega_{n} \rightarrow \mathfrak{M}_{n}(\mathcal{V})
$$

and

$$
f_{m+n} \mid \Omega_{m} \oplus \Omega_{n}=f_{m} \oplus f_{n}
$$

and

$$
\left(\operatorname{Ad} S \otimes I_{v}\right) \circ f_{n}=\left.f_{n} \circ\left(\operatorname{Ad} S \otimes I_{n}\right)\right|_{\Omega_{n}},
$$

when $S \in G L(n ; \mathbb{C})$.

\section{The Grassmannian completion}

3.1. For the affine theory it is sufficient to consider fully matricial functions and sets with respect to a Banach space with a specified non-zero element.

To construct the Grassmannian completion the Banach space must be upgraded to a Banach algebra over $\mathbb{C}$ with unit.

Throughout this section $B$ is a Banach algebra over $\mathbb{C}$ with unit $1 \in B$. The Grassmannian $G r_{n}(B)$ is defined to be the set

$$
G L_{2}\left(\mathfrak{M}_{n}(B)\right) / \widetilde{\lambda n}
$$


where

$$
\left(\begin{array}{ll}
a & b \\
c & d
\end{array}\right) \widetilde{\lambda n}\left(\begin{array}{ll}
a^{\prime} & b^{\prime} \\
c^{\prime} & d^{\prime}
\end{array}\right)
$$

if there is $t \in G L_{1}\left(\mathfrak{M}_{n}(B)\right)$ so that $b t=b^{\prime}, d t=d^{\prime}$. We may describe alternatively $G r_{n}(B)$ as the set of right $\mathfrak{M}_{n}(B)$ submodules of $\mathfrak{M}_{n}(B) \oplus$ $\mathfrak{M}_{n}(B)$ which are isomorphic to $\mathfrak{M}_{n}(B)$ and have a complement of the same kind. The submodule corresponding to $\left(\begin{array}{ll}a & b \\ c & d\end{array}\right) / \widetilde{\lambda n}$ is then

$$
\left\{\left(\begin{array}{c}
b \xi \\
d \xi
\end{array}\right) \in \mathfrak{M}_{n}(B) \oplus \mathfrak{M}_{n}(B) \mid \xi \in \mathfrak{M}_{n}(B)\right\} .
$$

Note also that the equivalence relation $\widetilde{\rho n}$ on $G L_{2}\left(\mathfrak{M}_{n}(B)\right)$

$$
\left(\begin{array}{ll}
a & b \\
c & d
\end{array}\right) \widetilde{\rho n}\left(\begin{array}{ll}
a^{\prime} & b^{\prime} \\
c^{\prime} & d^{\prime}
\end{array}\right)
$$

if at $=a^{\prime}$, ct $=c^{\prime}$ for some $t \in G L_{1}\left(\mathfrak{M}_{n}(B)\right)$, provides an alternative realization of $G r_{n}(B)$.

\subsection{If}

$$
\pi_{j}=\left(\begin{array}{cc}
a_{j} & b_{j} \\
c_{j} & d_{j}
\end{array}\right) / \widetilde{\lambda n_{j}} \in G r_{n_{j}}(B)
$$

$j=1,2$, we define

$$
\pi_{1} \oplus \pi_{2}=\left(\begin{array}{ll}
a_{1} \oplus a_{2} & b_{1} \oplus b_{2} \\
c_{1} \oplus c_{2} & d_{1} \oplus d_{2}
\end{array}\right) / \lambda\left(\widetilde{n_{1}+n_{2}}\right) \in G r_{n_{1}+n_{2}}(B) .
$$

Also if

$$
\pi=\left(\begin{array}{ll}
a & b \\
c & d
\end{array}\right) / \widetilde{\lambda n} \in G r_{n}(B)
$$

and $g \in G L_{2}\left(\mathfrak{M}_{n}(B)\right)$ we see that $g \pi=\left(g\left(\begin{array}{ll}a & b \\ c & d\end{array}\right)\right) / \lambda n$ is welldefined (i.e., depends only on $g$ and $\pi$ ). We will use extensively the action of $G L_{1}\left(\mathfrak{M}_{n}(\mathbb{C})\right)\left(\simeq G L_{n}(\mathbb{C})\right)$, denoted $s \cdot \pi$ where

$$
s \cdot \pi=\left(\begin{array}{cc}
s & 0 \\
0 & s
\end{array}\right) \pi
$$

and

$$
\left(\begin{array}{ll}
s & 0 \\
0 & s
\end{array}\right) \in G L_{2}\left(\mathfrak{M}_{n}(\mathbb{C})\right) \subset G L_{2}\left(\mathfrak{M}_{n}(B)\right)
$$

Clearly

$$
s \cdot \pi=\left(\begin{array}{ll}
s a & s b \\
s c & s d
\end{array}\right) / \widetilde{\lambda n}=\left(\begin{array}{ll}
s a s^{-1} & s b s^{-1} \\
s c s^{-1} & s d s^{-1}
\end{array}\right) / \widetilde{\lambda n}
$$


3.3. By definition a fully matricial $B$-set of the Grassmannian is a sequence of sets $\Omega=\left(\Omega_{n}\right)_{n \in \mathbb{N}}$ where $\Omega_{n} \subset G r_{n}(B)$ and the following two conditions are satisfied:

(i) $\Omega_{m+n} \cap\left(G r_{m}(B) \oplus G r_{n}(B)\right)=\Omega_{m} \oplus \Omega_{n}$

(ii) $s \cdot \Omega_{n}=\Omega_{n}$ if $s \in G L(n ; \mathbb{C})\left(=G L_{1}\left(\mathfrak{M}_{n}(\mathbb{C})\right)\right)$.

3.4. If $\Omega=\left(\Omega_{n}\right)_{n \in \mathbb{N}}$ is a fully matricial $B$-set of the Grassmannian and $\mathcal{U}$ is a Banach space over $\mathbb{C}$, then a fully matricial $\mathcal{U}$-valued function on $\Omega$ is a hierarchy of functions $f=\left(f_{n}\right)_{n \in \mathbb{N}}$ where $f_{n}: \Omega_{n} \rightarrow \mathfrak{M}_{n}(\mathcal{U})$ are such that:

(i) $f_{n_{1}+n_{2}}\left(\pi_{1} \oplus \pi_{2}\right)=f_{n_{1}}\left(\pi_{1}\right) \oplus f_{n_{2}}\left(\pi_{2}\right)$.

(ii) $f_{n}(s \cdot \pi)=s f_{n}(\pi) s^{-1}$ if $s \in G L(n ; \mathbb{C})$.

3.5. We can also define if $A, B$ are unital Banach algebras and if $\Omega, \Theta$ are fully matricial $A$ and respectively $B$ sets of the Grassmannian, fully matricial maps $F: \Omega \rightarrow \Theta$ as sequences $\left(F_{n}\right)_{n>1} F_{n}: \Omega_{n} \rightarrow \Theta_{n}$ satisfying $F_{n_{1}+n_{2}}\left(\pi_{1} \oplus \pi_{2}\right)=F_{n_{1}}\left(\pi_{1}\right) \oplus F_{n_{2}}\left(\pi_{2}\right)$ and $F_{n}(s \cdot \pi)=s \cdot F_{n}(\pi)$ $(s \in G L(n, \mathbb{C}))$.

3.6. To emphasize the distinction between fully matricial $B$-sets of the Grassmannian and fully matricial $B$-sets we will sometimes refer to the latter as affine fully matricial $B$-sets.

3.7. The Grassmannian $G r_{n}(B)$ is naturally a Banachic complex analytic manifold, so that a map defined on an open set $\omega$ of $G r_{n}(B)$ is analytic iff it is analytic when composed with the surjection

$$
p_{n}^{-1}(\omega) \rightarrow \omega
$$

where $p_{n}$ is the surjection

$$
G L_{2}\left(\mathfrak{M}_{n}(B) \rightarrow G r_{n}(B)\right.
$$

This can be checked along standard lines using charts

$$
\left\{\left(\left(\begin{array}{ll}
a & b \\
c & d
\end{array}\right)\left(\begin{array}{ll}
1 & f \\
0 & 1
\end{array}\right)\right) / \widetilde{\lambda n} \mid f \in \mathfrak{M}_{n}(B),\|f\|<\varepsilon\right\}
$$

for suitably small $\varepsilon>0$ (a reference for analytic functions on Banach space is [7]).

Remark that the largest affine fully matricial $B$-set $\left(\mathfrak{M}_{n}(B)\right)_{n \in \mathbb{N}}$ can be identified with a fully matricial $B$-set of the Grassmannian $\Omega=$ $\left(\Omega_{n}\right)_{n \in \mathbb{N}}$ where

$$
\Omega_{n}=\left\{\left(\begin{array}{ll}
1 & f \\
0 & 1
\end{array}\right) / \widetilde{\lambda n} \in G r_{n}(B) \mid f \in \mathfrak{M}_{n}(B)\right\} .
$$


We will call $\Omega_{n}$ the affine part of $G r_{n}(B)$. This identification also turns every affine fully matricial $B$-set into a fully matricial $B$-set of the Grassmannian. Fully matricial analytic maps of open affine matricial $B$-sets then become analytic fully matricial maps of the fully matricial $B$-set of the Grassmannian.

3.8. Note also, in a converse direction, that $\Omega=\left(\Omega_{n}\right)_{n \in \mathbb{N}}$ is a fully matricial $B$-set of the Grassmannian iff $p^{-1}(\Omega)=\left(p_{n}^{-1}\left(\Omega_{n}\right)\right)_{n \in \mathbb{N}}$ is an affine fully matricial $\mathfrak{M}_{2}(B)$-set. Here $p^{-1}(\Omega)$ is identified with a subset of $\left(G L_{n}\left(\mathfrak{M}_{2}(B)\right)\right)_{n \in \mathbb{N}}$ via the appropriate identifications

$$
G L_{n}\left(\mathfrak{M}_{2}(B)\right) \simeq G L_{2}\left(\mathfrak{M}_{n}(B)\right) .
$$

3.9. Note that for $K$-theory reasons for instance $G r_{n}(B)$ may not be connected and thus $\Omega_{n}$ the affine part of $G r_{n}(B)$ not only will not be dense in $G r_{n}(B)$ in this case, but even more it will not even be a set of uniqueness for analytic functions (consider for instance functions which are constant on the connected components). This occurs if for instance there is $u \in G L(2 ; B)$ such that $[u]$ its $K_{1}$-class is not in the subgroup $\left\{[v] \in K_{1}(B) \mid v \in G L(1 ; B)\right\}$. Indeed, since $G r_{2}(B)=G L(2 ; B) / T$ where $T=\left\{\left(\begin{array}{ll}a & 0 \\ b & d\end{array}\right) \in G L(2 ; B) \mid a, d \in G L(1, B)\right\}$ we will have that $u / \widetilde{\lambda 2}$ is not in the connected component of $\left(\begin{array}{ll}0 & 1 \\ 1 & 0\end{array}\right) / \widetilde{\lambda 2}$ in $G r_{2}(B)$.

Note also that if $\left(\begin{array}{ll}a & b \\ c & d\end{array}\right) \in G L(2 ; B)$ is such that $b$ is a nonunitary isometry, then $\left(\begin{array}{ll}a & b \\ c & d\end{array}\right) / \widetilde{\lambda 2}$ will not be in the closure of the affine part of $G r_{2}(B)$. If in $B$ the invertible elements are dense (i.e., $B$ has topological stable rank 1), then the affine part of $G r_{n}(B)$ is dense in $G r_{n}(B)$.

3.10. Let $B_{1}, \ldots, B_{p}$ be unital Banach algebras over $\mathbb{C}$. A fully multimatricial $\left.\left(B_{1}, \ldots, B_{p}\right)\right)$-set of the Grassmannians is a family of sets $\Omega=\left(\Omega_{n_{1}, \ldots, n_{p}}\right)_{\left(n_{1}, \ldots, n_{p}\right) \in \mathbb{N} p}$ where

$$
\Omega_{n_{1}, \ldots, n_{p}} \subset G r_{n_{1}}\left(B_{1}\right) \times \ldots \times G r_{n_{p}}\left(B_{p}\right)
$$

and for each $1 \leq j \leq p$ and $\pi_{k} \in G r_{n_{k}}\left(B_{k}\right) k \in\{1, \ldots, \hat{j}, \ldots, p\}$, $\left(\Omega_{n_{1}, \ldots, n_{j-1}, n, n_{j+1}, \ldots, n_{p}} \cap\left(\left\{\left(\pi_{1}, \ldots, \pi_{j-1}\right)\right\} \times G r_{n}\left(B_{j}\right) \times\left\{\left(\pi_{j+1}, \ldots, \pi_{p}\right)\right\}\right)\right)_{n \in \mathbb{N}}$ is a fully matricial $B_{j}$-set of the Grassmannian. A fully multimatricial $\mathcal{U}$-valued function on $\Omega$ is a family of functions $f=\left(f_{n_{1}, \ldots, n_{p}}\right)_{\left(n_{1}, \ldots, n_{p}\right) \in \mathbb{N}^{p}}$ where $f_{n_{1}, \ldots, n_{p}}: \Omega_{n_{1}, \ldots, n_{p}} \rightarrow \mathfrak{M}_{n_{1}} \otimes \cdots \otimes \mathfrak{M}_{n_{p}} \otimes \mathcal{U}$ and for each fixed 
$n_{1}, \ldots, n_{j-1}, n_{j+1}, \ldots, n_{p}$ and $\pi_{k}$ as above $f_{n_{1}, \ldots, n_{j-1}, n, n_{j+1}, \ldots, n_{p}}$ gives rise to a fully matricial

$$
\mathfrak{M}_{n_{1}} \otimes \cdots \otimes \mathfrak{M}_{n_{j-1}} \otimes \mathfrak{M}_{n_{j+1}} \otimes \cdots \otimes \mathfrak{M}_{n_{p}} \otimes \mathcal{U}
$$

valued functions on

$\left(\Omega_{n_{1}, \ldots, n_{j-1}, n, n_{j+1}, \ldots, n_{p}} \cap\left(\left\{\left(\pi_{1}, \ldots, \pi_{j-1}\right)\right\} \times G r_{n}\left(B_{j}\right) \times\left\{\left(\pi_{j+1}, \ldots, \pi_{p}\right)\right\}\right)\right)_{n \in \mathbb{N}}$.

\section{Grassmannian Resolvents and Resolvent Sets}

4.1. Let $E$ be a complex Banach algebra with 1 an $1 \in B \subset E$ a Banach subalgebra. Let further $\pi=\left(\begin{array}{ll}a & b \\ c & d\end{array}\right) / \widetilde{\lambda 1} \in G r_{1}(E)$. We define the $n$-th Grassmannian $B$-resolvent set of $\pi$ to be the set $\widetilde{\rho}_{n}(\pi ; B)=$ $\{\sigma \in G r_{n}(B) \mid \sigma$ is a complement of $\underbrace{\pi \oplus \cdots \oplus \pi}_{n \text {-times }}\}$. If $\sigma=\left(\begin{array}{ll}\alpha & \beta \\ \gamma & \delta\end{array}\right) / \widetilde{\lambda n}$ where $\alpha, \beta, \gamma, \delta \in \mathfrak{M}_{n}(B)$, then $\sigma \in \widetilde{\rho}_{n}(\pi ; B)$ is equivalent to requiring that

$$
\left(\begin{array}{cccc} 
& b & & O \\
\beta & & \ddots & \\
& O & & b \\
& d & & O \\
\delta & & \ddots & \\
& O & & d
\end{array}\right) \in G L(2 n ; E) .
$$

It is easily seen that $\widetilde{\rho}(\pi ; B)=\left(\widetilde{\rho}_{n}(\pi ; B)\right)_{n \in \mathbb{N}}$ is a fully matricial $B$-set of the Grassmannian. The direct sum property is obvious and the similarity property follows from the fact that $s \cdot(\underbrace{\pi \oplus \cdots \oplus \pi}_{n})=$ $\underbrace{\pi \oplus \cdots \oplus \pi}_{n}$ if $s \in G L(n ; \mathbb{C})$ and $\sigma$ is a complement of $\underbrace{\pi \oplus \cdots \oplus \pi}_{n}$ iff $s \cdot \sigma$ is a complement of $s \cdot(\pi \oplus \cdots \oplus \pi)$. We shall call $\widetilde{\rho}(\pi ; B)$ the full Grassmannian B-resolvent of $\pi$.

4.2. On $\widetilde{\rho}_{n}(\pi ; B)$ we define the $\mathfrak{M}_{n}(E)$-valued analytic function $\widetilde{\mathcal{R}}_{n}(\pi ; B)$. If $\sigma \in \widetilde{\rho}_{n}(\pi ; B)$ and $\pi$ are like in the preceding subsection, let $\zeta \in$ 
$\mathfrak{M}_{n}(E)$ be so that

$$
\left(\begin{array}{llll}
b & & O & \\
& \ddots & & \beta \\
O & & b & \\
d & & O & \\
& \ddots & & \delta \\
O & & d &
\end{array}\right)^{-1}=\left(\begin{array}{ll}
* & * \\
* & \zeta
\end{array}\right)
$$

Then we define $\widetilde{\mathcal{R}}_{n}(\pi ; B)(\sigma)=\beta \zeta$. If $\tau \in G L_{1}\left(\mathfrak{M}_{n}(B)\right), t \in G L_{1}(E)$, then replacing $\beta, \delta, b, d$ by $\beta \tau, \delta \tau, b t, d t$ will lead to replacing $\zeta$ by $\tau^{-1} \zeta$. Since $\beta \zeta=(\beta \tau)\left(\tau^{-1} \zeta\right)$ we see that $\widetilde{\mathcal{R}}_{n}(\pi ; B)(\sigma)$ is well-defined. We will call $\widetilde{\mathcal{R}}_{n}(\pi ; B)$ the $n$-th Grassmannian B-resolvent of $\pi$ and $\widetilde{\mathcal{R}}(\pi ; B)=$ $\left(\widetilde{\mathcal{R}}_{n}(\pi ; B)\right)_{n \in \mathbb{N}}$ the full Grassmannnian B-resolvent of $\pi$. It is easy to check that $\widetilde{\mathcal{R}}(\pi ; B)$ is a fully matricial E-valued analytic function on $\widetilde{\rho}(\pi ; B)$.

4.3. As a first step toward fitting the "affine" resolvents into this framework, we shall see what happens if $\pi$ is the graph of an element $Y \in E$, that is, if

$$
\pi=\left(\begin{array}{cc}
O & 1 \\
1 & Y
\end{array}\right) / \widetilde{\lambda 1} \in G r_{1}(E) .
$$

Remark that in this case

$$
\pi \oplus \cdots \oplus \pi=\left(\begin{array}{cc}
O & I_{n} \otimes 1 \\
I_{n} \otimes 1 & I_{n} \otimes Y
\end{array}\right) / \widetilde{\lambda n} .
$$

We shall denote the corresponding resolvents and resolvent sets with $\pi$ replaced by $Y$ and call them Grassmannian resolvents of $Y$, so that $\widetilde{\rho}_{n}(Y ; B)$ is the $n$-th Grassmannian resolvent set of $Y$ for instance.

Lemma. We have

$$
\left(\begin{array}{ll}
\alpha & \beta \\
\gamma & \delta
\end{array}\right) / \widetilde{\lambda n} \in \widetilde{\rho}_{n}(Y ; B)
$$

iff $\delta-\left(I_{n} \otimes Y\right) \beta$ is invertible.

Moreover, then

$$
\widetilde{\mathcal{R}}_{n}(Y ; B)\left(\left(\begin{array}{ll}
\alpha & \beta \\
\gamma & \delta
\end{array}\right) / \widetilde{\lambda n}\right)=\beta\left(\delta-\left(I_{n} \otimes Y\right) \beta\right)^{-1}
$$


Proof. The iff part follows from

$$
\left(\begin{array}{cc}
I_{n} \otimes 1 & \beta \\
I_{n} \otimes Y & \delta
\end{array}\right)=\left(\begin{array}{cc}
I_{n} \otimes 1 & 0 \\
I_{n} \otimes Y & \delta-\left(I_{n} \otimes Y\right) \beta
\end{array}\right)\left(\begin{array}{cc}
I_{n} \otimes 1 & \beta \\
0 & I_{n} \otimes 1
\end{array}\right) .
$$

The factorization also implies

$$
\left(\begin{array}{cc}
I_{n} \otimes 1 & \beta \\
I_{n} \otimes Y & \delta
\end{array}\right)^{-1}=\left(\begin{array}{cc}
* & * \\
* & \left(\delta-\left(I_{n} \otimes Y\right) \beta\right)^{-1}
\end{array}\right) .
$$

4.4. If $\sigma=\left(\begin{array}{cc}\alpha & I_{n} \otimes 1 \\ \gamma & \delta\end{array}\right) / \widetilde{\lambda n} \in \widetilde{\rho}_{n}(Y ; B)$ then $\widetilde{\mathcal{R}}_{n}(Y ; B)(\sigma)=(\delta-$ $\left.I_{n} \otimes Y\right)^{-1}$ and $\delta \in \rho_{n}(Y ; B)$ (the affine $n$-th resolvent). Thus the Grassmannian resolvent set and resolvent extend the affine ones.

4.5. Transversality. We will say that $\pi=\left(\begin{array}{ll}a & b \\ c & d\end{array}\right) / \widetilde{\lambda n}$ and $\pi^{\prime}=$ $\left(\begin{array}{ll}a^{\prime} & b^{\prime} \\ c^{\prime} & d^{\prime}\end{array}\right) / \widetilde{\lambda n}$ in $G r_{n}(B)$ are transversal if $\left(\begin{array}{ll}b^{\prime} & b \\ d^{\prime} & d\end{array}\right)$ is invertible. It is easily seen that the relation is symmetric and well-defined (i.e., depends only on the equivalence classes $/ \widetilde{\lambda n})$. Clearly, in terms of transversality, if $\theta \in G r_{1}(E)$ and $\sigma \in G r_{n}(B)$ then $\sigma \in \widetilde{\rho}_{n}(\theta ; B)$ means precisely that $\sigma$ and $\theta \oplus \cdots \oplus \theta$ are transversal. We will frequently use in this paper the following rather obvious fact.

Lemma. Let $\pi, \pi^{\prime} \in G r_{n}(B)$ and $g \in G L_{2}\left(\mathfrak{M}_{n}(B)\right)$. Then $\pi, \pi^{\prime}$ are transversal iff $g \pi$ and $g \pi^{\prime}$ are transversal.

Corollary. Let $\gamma \in G L_{2}(B), g=I_{n} \otimes \gamma \in G L_{2}\left(\mathfrak{M}_{n}(B)\right)$ and $\pi \in$ $G r_{1}(E)$. Then we have $\widetilde{\rho}_{n}(\gamma \pi ; B)=g \widetilde{\rho}_{n}(\pi ; B)$.

\section{The Derivation $\widetilde{\partial}$ On Fully matricial FunCtions of the GRASSMANNIAN}

5.1. Let $\Omega$ be a fully matricial open $B$-set of the Grassmannian. We shall denote by $\mathcal{A}(\Omega)$ the algebra of $\mathbb{C}$-valued (that is scalar) fully matricial analytic functions on $\Omega$, under pointwise multiplication of the matricial values. More generally we get an algebra $\mathcal{A}(\Omega)$ for a fully multimatricial $\left(B_{1}, \ldots, B_{p}\right)$-set of the Grassmannians. In particular, if $\Omega$ is a fully matricial open $B$-set of the Grassmannian, then $\Omega \times \Omega$ is a fully multimatricial $(B, B)$-set and we shall denote the corresponding algebra by $\mathcal{A}(\Omega ; \Omega)$. More generally we have algebras $\mathcal{A}(\Omega ; \ldots ; \Omega)$. This extends the construction in the affine case [16]. The aim of this section will be to extend the construction of the derivation $\partial$ from the 
affine case to a derivation $\widetilde{\partial}$ in the Grassmannian framework. Like in the affine case the construction rests on two technical lemmas.

5.2. Lemma. Let $\Omega=\left(\Omega_{n}\right)_{n \in \mathbb{N}}$ be an open fully matricial $B$-set of the Grassmannian and let

$$
\left(\begin{array}{cc}
a_{j} & b_{j} \\
c_{j} & d_{j}
\end{array}\right) / \widetilde{\lambda n_{j}} \in \Omega_{n_{j}} \quad(j=1,2) .
$$

Then for all $x, y, z, t \in \mathfrak{M}_{n_{1}, n_{2}}(B)$

$$
\left(\begin{array}{cccc}
a_{1} & x & b_{1} & y \\
0 & a_{2} & 0 & b_{2} \\
c_{1} & z & d_{1} & t \\
0 & c_{2} & 0 & d_{2}
\end{array}\right) / \widehat{\lambda} \widetilde{n_{1}+n_{2}} \in \Omega_{n_{1}+n_{2}} .
$$

Proof. Since $\Omega$ is open, for any given $x, y, z, t$ there is $\varepsilon \neq 0$ so that the conclusion of the lemma holds with $x, y, z, t$ replaced by $\varepsilon x, \varepsilon y, \varepsilon z, \varepsilon t$. To obtain the result without $\varepsilon$, it suffices to use the $G L\left(n_{1}+n_{2} ; \mathbb{C}\right)$ invariance with $s=\left(\begin{array}{cc}I_{n_{1}} & 0 \\ 0 & \varepsilon I_{n_{2}}\end{array}\right)$.

5.3. Lemma. Let $\Omega$ be an open fully matricial B-set of the Grassmannian and $f \in \mathcal{A}(\Omega)$ and let $a_{j}, b_{j}, c_{j}, d_{j}, t$ be like in the preceding lemma. Then, there is $k \in \mathfrak{M}_{n_{1}, n_{2}}(\mathbb{C})$ so that

$$
\begin{aligned}
& f_{n_{1}+n_{2}}\left(\left(\begin{array}{cccc}
a_{1} & 0 & b_{1} & 0 \\
0 & a_{2} & 0 & b_{2} \\
c_{1} & 0 & d_{1} & t \\
0 & c_{2} & 0 & d_{2}
\end{array}\right) / \widetilde{\lambda n_{1}+n_{2}}\right) \\
& =\left(\begin{array}{cc}
f_{n_{1}}\left(\left(\begin{array}{cc}
a_{1} & b_{1} \\
c_{1} & d_{1}
\end{array}\right) / \widetilde{\lambda n_{1}}\right) & k \\
0 & f_{n_{2}}\left(\left(\begin{array}{cc}
a_{2} & b_{2} \\
c_{2} & d_{2}
\end{array}\right) / \widetilde{\lambda n_{2}}\right)
\end{array}\right)
\end{aligned}
$$

and $k$ depends linearly on $t$. In fact we have

$$
\left(\begin{array}{ll}
0 & k \\
0 & 0
\end{array}\right)=\left.\frac{d}{d \varepsilon} f_{n_{1}+n_{2}}\left(\left(\begin{array}{cccc}
a_{1} & 0 & b_{1} & 0 \\
0 & a_{2} & 0 & b_{2} \\
c_{1} & 0 & d_{1} & \varepsilon t \\
0 & c_{2} & 0 & d_{2}
\end{array}\right) / \widehat{\lambda n_{1}+n_{2}}\right)\right|_{\varepsilon=0} .
$$


Proof. Assume the right-hand side of the first equality is $\left(\begin{array}{ll}u & k \\ h & v\end{array}\right)$. Then by the $G L\left(n_{1}+n_{2} ; \mathbb{C}\right)$ equivariance of $f_{n_{1}+n_{2}}$ applied to the similarity $\left(\begin{array}{cc}\varepsilon I_{n_{1}} & 0 \\ 0 & I_{n_{2}}\end{array}\right)$ we find that $\left(\begin{array}{cc}u & \varepsilon k \\ \varepsilon^{-1} h & v\end{array}\right)$ converges as $\varepsilon \rightarrow 0$ to $\left(\begin{array}{cc}f_{n_{1}}\left(\left(\begin{array}{ll}a_{1} & b_{1} \\ c_{1} & d_{1}\end{array}\right) / \lambda n_{1}\right) & \begin{array}{c}0 \\ 0\end{array} \\ f_{n_{2}}\left(\left(\begin{array}{ll}a_{2} & b_{2} \\ c_{2} & d_{2}\end{array}\right) / \lambda n_{2}\right)\end{array}\right)$. This, then, implies $h=0$ and that $\left(\begin{array}{ll}0 & k \\ 0 & 0\end{array}\right)$ is given by the second formula in the statement of the lemma, since $f$ as an analytic function is differentiable. In turn, this formula which identifies the map taking $t$ to $k$ with a partial differential of $f_{n_{1}+n_{2}}$ shows that this map is a $\mathbb{C}$-linear map.

5.4. To define $\widetilde{\partial}_{n_{1}, n_{2}} f_{n_{1}+n_{2}}$, we shall use the isomorphism

$$
\alpha_{n_{1}, n_{2}}: \mathfrak{M}_{n_{1}} \otimes \mathfrak{M}_{n_{2}} \rightarrow \mathcal{L}\left(\mathfrak{M}_{n_{1}, n_{2}}\right)
$$

which takes $A \otimes B$ to the linear map $X \rightarrow A X B$ in $\mathcal{L}\left(\mathfrak{M}_{n_{1}, n_{2}}\right)$.

Definition. Let $\Omega_{1}, f, a_{j}, b_{j}, c_{j}, d_{j}$ be like in 5.3 and let $T \in \mathcal{L}\left(\mathfrak{M}_{n_{1}, n_{2}}\right)$ be the linear map, so that $T(t)=k$ when $t \in \mathfrak{M}_{n_{1}, n_{2}}(\mathbb{C}) \subset \mathfrak{M}_{n_{1}, n_{2}}(B)$ and

$$
\left(\begin{array}{ll}
0 & k \\
0 & 0
\end{array}\right)=\frac{d}{d \varepsilon} f_{n_{1}+n_{2}}\left(\left(\begin{array}{cccc}
a_{1} & 0 & b_{1} & 0 \\
0 & a_{2} & 0 & b_{2} \\
c_{1} & 0 & d_{1} & \varepsilon t b_{2} \\
0 & c_{2} & 0 & d_{2}
\end{array}\right) /\left.\widetilde{\lambda n_{1}+n_{2}}\right|_{\varepsilon=0} .\right.
$$

Then we define

$$
\begin{aligned}
& \left(\widetilde{\partial}_{n_{1}, n_{2}} f_{n_{1}+n_{2}}\right)\left(\left(\begin{array}{ll}
a_{1} & b_{1} \\
c_{1} & d_{1}
\end{array}\right) / \widetilde{\lambda n_{1}} ;\left(\begin{array}{cc}
a_{2} & b_{2} \\
c_{2} & d_{2}
\end{array}\right) / \widetilde{\lambda n_{2}}\right) \\
& =\alpha_{n_{1}, n_{2}}^{-1}(T) \in \mathfrak{M}_{n_{1}} \otimes \mathfrak{M}_{n_{2}} .
\end{aligned}
$$

Note that if $z_{j} \in G L_{1}\left(\mathfrak{M}_{n_{j}}(B)\right)$ then $\left(\begin{array}{cc}z_{1} & 0 \\ 0 & z_{2}\end{array}\right) \in G L_{1}\left(\mathfrak{M}_{n_{1}+n_{2}}(B)\right)$ and

$$
f_{n_{1}+n_{2}}\left(\left(\begin{array}{cccc}
a_{1} & 0 & b_{1} z_{1} & 0 \\
0 & a_{2} & 0 & b_{2} z_{2} \\
c_{1} & 0 & d_{1} z_{1} & \varepsilon t b_{2} z_{2} \\
0 & c_{2} & 0 & d_{2} z_{2}
\end{array}\right) / \lambda n_{1}+n_{2}\right)
$$




$$
=f_{n_{1}+n_{2}}\left(\left(\begin{array}{cccc}
a_{1} & 0 & b_{1} & 0 \\
0 & a_{2} & 0 & b_{2} \\
c_{1} & 0 & d_{1} & \varepsilon t b_{2} \\
0 & c_{2} & 0 & d_{2}
\end{array}\right) / \lambda n_{1}+n_{2}\right)
$$

so that $\widetilde{\partial}_{n_{1}, n_{2}} f_{n_{1}+n_{2}}$ is well-defined.

It is also easy to see that $\widetilde{\partial}$ extends the definition of $\partial$ in the affine case $([16])$. Indeed if we take $a_{j}=I_{n_{j}} \otimes 1, c_{j}=0, b_{j}=I_{n_{j}} \otimes 1$ in the preceding formulae we get exactly the formulae in the affine case, corresponding to the embedding

$$
\mathfrak{M}_{n}(B) \ni \beta \rightarrow\left(\begin{array}{cc}
I_{n} \otimes 1 & I_{n} \otimes 1 \\
0 & \beta
\end{array}\right) / \lambda n \in G r_{n}(B) .
$$

5.5. Starting with this subsection and continuing in 5.6 and 5.7 we will check that $\widetilde{\partial}$ turns $A(\Omega)$ into a "topological" infinitesimal bialgebra. Since sections 5.5-5.7 are just a technical extension of the affine case (sections 7.7-7.10 in [16]), our exposition will be more compressed.

The first step is to check that

$$
\widetilde{\partial} f=\left(\widetilde{\partial}_{m, n} f_{m+n}\right)_{(m, n) \in \mathbb{N}^{2}} \in \mathcal{A}(\Omega ; \Omega) .
$$

Since analyticity of the $\widetilde{\partial}_{m, n} f_{m+n}$ is obvious, we are left with checking $G L(m) \times G L(n)$ equivariance and the direct sum properties.

In view of the equivariance property of $\alpha_{m, n}$ (see 7.7 in [16]) it suffices to remark that if $S^{\prime} \in G L(m)$ and $S^{\prime \prime} \in G L(n)$ then assuming $t, k \in$ $\mathfrak{M}_{m, n}$ and

$$
\left(\begin{array}{cc}
0 & k \\
0 & 0
\end{array}\right)=\left.\frac{d}{d \varepsilon} f_{m+n}\left(\left(\begin{array}{cccc}
a_{1} & 0 & b_{1} & 0 \\
0 & a_{2} & 0 & b_{2} \\
c_{1} & 0 & d_{1} & \varepsilon t b_{2} \\
0 & c_{2} & 0 & d_{2}
\end{array}\right) / \widetilde{\lambda m+n}\right)\right|_{\varepsilon=0}
$$

we also have

$$
\begin{aligned}
& \left(\begin{array}{cc}
0 & S^{\prime} k S^{\prime \prime-1} \\
0 & 0
\end{array}\right) \\
& =\left.\frac{d}{d \varepsilon} f_{m+n}\left(\left(\begin{array}{cccc}
S^{\prime} a_{1} S^{\prime-1} & 0 & S^{\prime} b_{1} S^{\prime-1} & 0 \\
0 & S^{\prime \prime} a_{2} S^{\prime \prime-1} & 0 & S^{\prime \prime} b_{2} S^{\prime \prime-1} \\
S^{\prime} c_{1} S^{\prime-1} & 0 & S^{\prime} d_{1} S^{\prime-1} & \varepsilon\left(S^{\prime} t S^{\prime \prime}-1\right)\left(S^{\prime \prime} b_{2} S^{\prime \prime-1}\right) \\
0 & S^{\prime \prime} c_{2} S^{\prime \prime-1} & 0 & S^{\prime \prime} d_{2} S^{\prime \prime-1}
\end{array}\right) / \widetilde{\lambda m+n}\right)\right|_{\varepsilon=0} .
\end{aligned}
$$

The last equality is a consequence of the $G L(m+n)$ equivalence of $f_{m+n}$ applied to $\left(\begin{array}{cc}S^{\prime} & 0 \\ 0 & S^{\prime \prime}\end{array}\right)$. We thus have proved that $\widetilde{\partial}_{m, n} f_{m+n}$ satisfies $G L(m) \times G L(n)$ equivariance. 
The direct sum properties to be checked are: if $\pi \in \Omega_{m}, \sigma \in \Omega_{n}$ and

$$
\begin{aligned}
m & =m^{\prime}+m^{\prime \prime}, \pi^{\prime} \in \Omega_{m^{\prime}}, \pi^{\prime \prime} \in \Omega_{m^{\prime \prime}} \\
n & =n^{\prime}+n^{\prime \prime}, \quad \sigma^{\prime} \in \Omega_{n^{\prime}}, \quad \sigma^{\prime \prime} \in \Omega_{n^{\prime \prime}}
\end{aligned}
$$

then

$$
\begin{aligned}
& \left(\widetilde{\partial}_{m, n} f_{m+n}\right)\left(\pi^{\prime} \oplus \pi^{\prime \prime}, \sigma\right)=\widetilde{\partial}_{m^{\prime}, n} f_{m^{\prime}+n}\left(\pi^{\prime}, \sigma\right) \oplus \widetilde{\partial}_{m^{\prime \prime}, n} f_{m^{\prime \prime}+n}\left(\pi^{\prime \prime}, \sigma\right) \\
& \left(\widetilde{\partial}_{m, n} f_{m+n}\right)\left(\pi, \sigma^{\prime} \oplus \sigma^{\prime \prime}\right)=\widetilde{\partial}_{m, n^{\prime}} f_{m+n^{\prime}}\left(\pi, \sigma^{\prime}\right) \oplus \widetilde{\partial}_{m, n^{\prime \prime}} f_{m+n^{\prime \prime}}\left(\pi, \sigma^{\prime \prime}\right) .
\end{aligned}
$$

We will only discuss the first equality to be checked, the second being obtainable along similar lines.

Since the isomorphism $\alpha$ has the property:

$$
\alpha_{m^{\prime}+m^{\prime \prime}, n}^{-1}\left(T_{1} \oplus T_{2}\right)=\alpha_{m^{\prime}, n}^{-1}\left(T_{1}\right) \oplus \alpha_{m^{\prime \prime}, n}\left(T_{2}\right)
$$

if $T_{1} \in \mathcal{L}\left(\mathfrak{M}_{m^{\prime}, n}\right), T_{2} \in \mathcal{L}\left(\mathfrak{M}_{m^{\prime \prime}, n}\right)$ it is easily seen that what we must prove boils down to the following.

We have

$$
\begin{aligned}
& f_{m^{\prime}+m^{\prime \prime}+n}\left(\left(\begin{array}{cccccc}
a_{1}^{\prime} & 0 & 0 & \beta_{1}^{\prime} & 0 & 0 \\
0 & a_{1}^{\prime \prime} & 0 & 0 & b_{1}^{\prime \prime} & 0 \\
0 & 0 & a_{2} & 0 & 0 & b_{2} \\
& & & & & \\
c_{1}^{\prime} & 0 & 0 & d_{1}^{\prime} & 0 & t^{\prime} \\
0 & c_{1}^{\prime \prime} & 0 & 0 & d_{1}^{\prime \prime} & t^{\prime \prime} \\
0 & 0 & c_{2} & 0 & 0 & d_{2}
\end{array}\right) / \lambda m^{\prime}+m^{\prime \prime}+n\right) \\
& =\left(\begin{array}{ccc}
f_{m^{\prime}}\left(\left(\begin{array}{cc}
a_{1}^{\prime} & b_{1}^{\prime} \\
c_{1}^{\prime} & d_{1}^{\prime}
\end{array}\right) / \widetilde{\lambda m^{\prime}}\right) & k^{\prime} \\
0 & f_{m^{\prime \prime}}\left(\left(\begin{array}{cc}
a_{1}^{\prime \prime} & b_{1}^{\prime \prime} \\
c_{1}^{\prime \prime} & d_{1}^{\prime \prime}
\end{array}\right) / \widetilde{\lambda m^{\prime \prime}}\right) & k^{\prime \prime} \\
0 & 0 & f_{n}\left(\left(\begin{array}{ll}
a_{2} & b_{2} \\
c_{2} & d_{2}
\end{array}\right) / \widetilde{\lambda n}\right)
\end{array}\right)
\end{aligned}
$$

where

$$
\begin{aligned}
& f_{m^{\prime}+n}\left(\left(\begin{array}{cccc}
a_{1}^{\prime} & 0 & b_{1}^{\prime} & 0 \\
0 & a_{2} & 0 & b_{2} \\
c_{1}^{\prime} & 0 & d_{1}^{\prime} & t^{\prime} \\
0 & c_{2} & 0 & d_{2}
\end{array}\right) / \widetilde{\lambda m^{\prime}+n}\right) \\
& =\left(\begin{array}{cc}
f_{m^{\prime}}\left(\left(\begin{array}{ll}
a_{1}^{\prime} & b_{1}^{\prime} \\
c_{1}^{\prime} & d_{1}^{\prime}
\end{array}\right) / \widetilde{\lambda m^{\prime}}\right) & k^{\prime} \\
0 & f_{n}\left(\left(\begin{array}{ll}
a_{2} & b_{2} \\
c_{2} & d_{2}
\end{array}\right) / \widetilde{\lambda n}\right)
\end{array}\right)
\end{aligned}
$$


and

$$
\begin{aligned}
& f_{m^{\prime \prime}+n}\left(\left(\begin{array}{cccc}
a_{1}^{\prime \prime} & 0 & b_{1}^{\prime \prime} & 0 \\
0 & a_{2} & 0 & b_{2} \\
c_{1}^{\prime \prime} & 0 & d_{1}^{\prime \prime} & t^{\prime \prime} \\
0 & c_{2} & 0 & d_{2}
\end{array}\right) / \widehat{\lambda m^{\prime \prime}+n}\right) \\
& \left.=\left(\begin{array}{cc}
f_{m^{\prime \prime}}\left(\left(\begin{array}{ll}
a_{1}^{\prime \prime} & b_{1}^{\prime \prime} \\
c_{1}^{\prime \prime} & d_{1}^{\prime \prime}
\end{array}\right) / \widetilde{\lambda m^{\prime \prime}}\right) & k^{\prime \prime} \\
0 & f_{n}\left(\left(\begin{array}{ll}
a_{2} & b_{2} \\
c_{2} & d_{2}
\end{array}\right) / \widetilde{\lambda n}\right.
\end{array}\right)\right) .
\end{aligned}
$$

If we define $k^{\prime}$ and $k^{\prime \prime}$ by the last two equalities (with Lemma 5.3 in mind) we get

$$
\begin{aligned}
& f_{m^{\prime}+m^{\prime \prime}+n}\left(\left(\begin{array}{cccccc}
a_{1}^{\prime} & 0 & 0 & b_{1}^{\prime} & 0 & 0 \\
0 & a_{1}^{\prime \prime} & 0 & 0 & b_{1}^{\prime \prime} & 0 \\
0 & 0 & a_{2} & 0 & 0 & b_{2} \\
c_{1}^{\prime} & 0 & 0 & d_{1}^{\prime} & 0 & t^{\prime} \\
0 & c_{1}^{\prime \prime} & 0 & 0 & d_{1}^{\prime \prime} & t^{\prime \prime} \\
0 & 0 & c_{2} & 0 & 0 & d_{2}
\end{array}\right) / \lambda m^{\prime} \widetilde{+m^{\prime \prime}}+n\right) \\
& \left.=\left(\begin{array}{ccc}
f_{m^{\prime}}\left(\left(\begin{array}{ll}
a_{1}^{\prime} & b_{1}^{\prime} \\
c_{1}^{\prime} & d_{1}^{\prime}
\end{array}\right) / \widetilde{\lambda m^{\prime}}\right) & * & * \\
0 & f_{m^{\prime \prime}}\left(\left(\begin{array}{ll}
a_{1}^{\prime \prime} & b_{1}^{\prime \prime} \\
c_{1}^{\prime \prime} & d_{1}^{\prime \prime}
\end{array}\right) / \widetilde{\lambda m^{\prime \prime}}\right) & k^{\prime \prime} \\
0 & 0 & f_{n}\left(\left(\begin{array}{ll}
a_{2} & b_{2} \\
c_{2} & d_{2}
\end{array}\right) / \widetilde{\lambda n}\right.
\end{array}\right)\right)
\end{aligned}
$$

and

$$
\begin{aligned}
& f_{m^{\prime \prime}+m^{\prime}+n}\left(\left(\begin{array}{cccccc}
a_{1}^{\prime \prime} & 0 & 0 & b_{1}^{\prime \prime} & 0 & 0 \\
0 & a_{1}^{\prime} & 0 & 0 & b_{1}^{\prime} & 0 \\
0 & 0 & a_{2} & 0 & 0 & b_{2} \\
c_{1}^{\prime \prime} & 0 & 0 & d_{1}^{\prime \prime} & 0 & t^{\prime \prime} \\
0 & c_{1}^{\prime} & 0 & 0 & d_{1}^{\prime} & t^{\prime} \\
0 & 0 & c_{2} & 0 & 0 & d_{2}
\end{array}\right) / \lambda m^{\prime \prime}+m^{\prime}+n\right) \\
& =\left(\begin{array}{ccc}
f_{m^{\prime \prime}}\left(\left(\begin{array}{ll}
a_{1}^{\prime \prime} & b_{1}^{\prime \prime} \\
c_{1}^{\prime \prime} & d_{1}^{\prime \prime}
\end{array}\right) / \widetilde{\lambda m^{\prime \prime}}\right) & * & * \\
0 & f_{m^{\prime}}\left(\left(\begin{array}{cc}
a_{1}^{\prime} & b_{1}^{\prime} \\
c_{1}^{\prime} & d_{1}^{\prime}
\end{array}\right) / \widetilde{\lambda m^{\prime}}\right) & k^{\prime} \\
0 & 0 & f_{n}\left(\left(\begin{array}{ll}
a_{2} & b_{2} \\
c_{2} & d_{2}
\end{array}\right) / \widetilde{\lambda n}\right)
\end{array}\right) .
\end{aligned}
$$

Using a similarity which permutes the first two summands in $\mathbb{C}^{m^{\prime}} \oplus$ $\mathbb{C}^{m^{\prime \prime}} \oplus \mathbb{C}^{n}$, we get that the 13 -block in the formula for $f_{m^{\prime}+m^{\prime \prime}+n}(\ldots)$ 
is $k^{\prime}$. Thus all we must still do is to show that the 12 -block in that formula is zero. This in turn is immediate from Lemma 5.3 applied to $f\left(m^{\prime}+m^{\prime \prime}\right)+n$ and $f_{m^{\prime}+m^{\prime \prime}}$. Thus we concluded checking that

$$
\left(\widetilde{\partial}_{m, n} f_{m+n}\right)_{(m, n) \in \mathbb{N}^{2}} \in \mathcal{A}(\Omega ; \Omega) .
$$

5.6. Our next task is to show that $\widetilde{\partial}: \mathcal{A}(\Omega) \rightarrow \mathcal{A}(\Omega ; \Omega)$ is a derivation.

Lemma. Let $f, g \in \mathcal{A}(\Omega)$ and let $\pi^{\prime}=\left(\begin{array}{ll}a^{\prime} & b^{\prime} \\ c^{\prime} & d^{\prime}\end{array}\right) / \widetilde{\lambda m} \in \Omega_{m}, \pi^{\prime \prime}=$ $\left(\begin{array}{ll}a^{\prime \prime} & b^{\prime \prime} \\ c^{\prime \prime} & d^{\prime \prime}\end{array}\right) / \widetilde{\lambda n} \in \Omega_{n}$ and $t \in \mathfrak{M}_{m, n}$. Then we have

$$
\begin{aligned}
\alpha_{m, n}\left(\left(\widetilde{\partial}_{m, n}(f g)_{m+n}\right)\left(\pi^{\prime} ; \pi^{\prime \prime}\right)\right)(t) & =f_{m}\left(\pi^{\prime}\right) \alpha_{m, n}\left(\left(\widetilde{\partial}_{m, n} g_{m+n}\right)\left(\pi^{\prime} ; \pi^{\prime \prime}\right)\right)(t) \\
& +\alpha_{m, n}\left(\left(\widetilde{\partial}_{m, n} f_{m+n}\right)\left(\pi^{\prime} ; \pi^{\prime \prime}\right)\right)(t) g_{n}\left(\pi^{\prime \prime}\right) .
\end{aligned}
$$

Proof. To simplify notations put

$$
\begin{aligned}
& \xi=\alpha_{m, n}\left(\left(\widetilde{\partial}_{m, n} f_{m+n}\right)\left(\pi^{\prime} ; \pi^{\prime \prime}\right)\right)(t) \in \mathfrak{M}_{m, n}, \\
& \eta=\alpha_{m, n}\left(\left(\widetilde{\partial}_{m, n} g_{m+n}\right)\left(\pi^{\prime} ; \pi^{\prime \prime}\right)\right)(t) \in \mathfrak{M}_{m, n}, \\
& \zeta=\alpha_{m, n}\left(\left(\widetilde{\partial}_{m, n}(f g)_{m+n}\right)\left(\pi^{\prime} ; \pi^{\prime \prime}\right)\right)(t) \in \mathfrak{M}_{m, n}
\end{aligned}
$$

and

$$
\pi=\left(\begin{array}{cccc}
a^{\prime} & 0 & b^{\prime} & 0 \\
0 & a^{\prime \prime} & 0 & b^{\prime \prime} \\
c^{\prime} & 0 & d^{\prime} & t b^{\prime \prime} \\
0 & c^{\prime} & 0 & d^{\prime \prime}
\end{array}\right) / \widehat{\lambda m+n} \in \Omega_{m+n}
$$

Then, by Lemma 5.3 and Definition 5.4 we have

$$
\begin{gathered}
(f g)_{m+n}(\pi)=\left(\begin{array}{cc}
f_{m}\left(\pi^{\prime}\right) g_{m}\left(\pi^{\prime}\right) & \zeta \\
0 & f_{m}\left(\pi^{\prime \prime}\right) g_{n}\left(\pi^{\prime \prime}\right)
\end{array}\right) \\
f_{m+n}(\pi)\left(\begin{array}{cc}
f_{m}\left(\pi^{\prime}\right) & \xi \\
0 & f_{n}\left(\pi^{\prime \prime}\right)
\end{array}\right)
\end{gathered}
$$

and

$$
g_{m+n}(\pi)=\left(\begin{array}{cc}
g_{m}\left(\pi^{\prime}\right) & \eta \\
0 & g_{n}\left(\pi^{\prime \prime}\right)
\end{array}\right) .
$$

The lemma then follows from the equality of matrices derived from

$$
(f g)_{m+n}(\pi)=f_{m+n}(\pi) g_{m+n}(\pi) .
$$

Corollary. $\widetilde{\partial}: \mathcal{A}(\Omega) \rightarrow \mathcal{A}(\Omega ; \Omega)$ is a derivation. 
Proof. Take into account that if $f, g \in \mathcal{A}(\Omega)$ and $h \in \mathcal{A}(\Omega ; \Omega)$ then the $\mathcal{A}(\Omega)$-bimodule structure $\mathcal{A}(\Omega ; \Omega)$ is given by the homomorphisms $f \rightarrow f \otimes 1$ and $g \rightarrow 1 \otimes g$ where $(f \otimes 1)_{m, n}\left(\pi^{\prime}, \pi^{\prime \prime}\right)=f_{m}\left(\pi^{\prime}\right) \otimes I_{n}$, $(1 \otimes g)_{m, n}\left(\pi^{\prime}, \pi^{\prime \prime}\right)=I_{m} \otimes g_{n}\left(\pi^{\prime \prime}\right)$, and that if $A \in \mathfrak{M}_{m}, B \in \mathfrak{M}_{n}$, $T \in \mathcal{L}\left(\mathfrak{M}_{m, n}\right)$ then

$$
\alpha_{m, n}^{-1}(A T(\cdot) B)=\left(A \otimes I_{n}\right) \alpha_{m, n}^{-1}(T(\cdot))\left(I_{m} \otimes B\right),
$$

the Corollary is immediately inferred from the Lemma.

5.7. We pass now to the proof of the co-associativity property of $\widetilde{\partial}$. Like in the affine case (7.10 in [16]) since $\mathcal{A}(\Omega ; \Omega)$ and $\mathcal{A}(\Omega ; \Omega ; \Omega)$ have not been identified with some topological tensor products of two and respectively three copies of $\mathcal{A}(\Omega)$, we will have to define the maps id $\otimes \widetilde{\partial}$ : $\mathcal{A}(\Omega ; \Omega) \rightarrow \mathcal{A}(\Omega ; \Omega ; \Omega)$ and $\widetilde{\partial} \otimes$ id $: \mathcal{A}(\Omega ; \Omega) \rightarrow \mathcal{A}(\Omega ; \Omega ; \Omega)$.

Let $\widetilde{k} \in \mathcal{A}(\Omega ; \Omega)$ and put $k=\widetilde{k}_{m, n+p}$, which is an analytic function on $\Omega_{m} \times \Omega_{n+p}$ with values in $\mathfrak{M}_{m} \otimes \mathfrak{M}_{n+p}$. Let further $\pi=\left(\begin{array}{ll}a & b \\ c & d\end{array}\right) / \widetilde{\lambda m} \in$ $\Omega_{m}, \pi^{\prime}=\left(\begin{array}{ll}a^{\prime} & b^{\prime} \\ c^{\prime} & d^{\prime}\end{array}\right) / \widetilde{\lambda n} \in \Omega_{n}, \pi^{\prime \prime}=\left(\begin{array}{ll}a^{\prime \prime} & b^{\prime \prime} \\ c^{\prime \prime} & d^{\prime \prime}\end{array}\right) / \widetilde{\lambda p} \in \Omega_{p}$. We define:

$\left((\mathrm{id} \otimes \widetilde{\partial})_{m, n, p} k\right)\left(\pi ; \pi^{\prime} ; \pi^{\prime \prime}\right)$

$$
\begin{aligned}
= & \sum_{\substack{1 \leq a, b \leq m \\
1 \leq c, d \leq n \\
1 \leq e, f \leq p}}\left(\left.\frac{d}{d \varepsilon} k\left(\pi ;\left(\begin{array}{cccc}
a^{\prime} & 0 & b^{\prime} & 0 \\
0 & a^{\prime \prime} & 0 & b^{\prime \prime} \\
c^{\prime} & 0 & d^{\prime} & \varepsilon\left(e_{d, e} \otimes 1\right) b^{\prime \prime} \\
0 & c^{\prime \prime} & 0 & d^{\prime \prime}
\end{array}\right) / \widetilde{\lambda n+p}\right)\right|_{\substack{(m)\\
}} e_{a b}^{(m)} \otimes e_{c d}^{(n)} \otimes e_{e f}^{(p)}\right.
\end{aligned}
$$

where $e_{i j}^{(r)}$ are the matrix-units in $\mathfrak{M}_{r}$ and the index $(a, b)(c, n+f)$ indicates the coefficient of $e_{a b}^{(m)} \otimes e_{c, n+f}^{(n+p)}$ of an element of $\mathfrak{M}_{m} \otimes \mathfrak{M}_{n+p}$. It is easy to see that if $\widetilde{k}=f \otimes g$, where $f, g \in \mathcal{A}(\Omega)$ then $(\mathrm{id} \otimes \widetilde{\partial})(f \otimes g)=$ $f \otimes \widetilde{\partial} g$. We also leave it to the reader to check that id $\otimes \widetilde{\partial}$ takes values in $\mathcal{A}(\Omega ; \Omega ; \Omega)$. This involves arguments of the type used in showing that $\widetilde{\partial}$ takes values in $\mathcal{A}(\Omega ; \Omega)$.

Similarly, we define

$\left((\widetilde{\partial} \otimes \mathrm{id})_{m, n, p} k\right)\left(\pi ; \pi^{\prime} ; \pi^{\prime \prime}\right)$

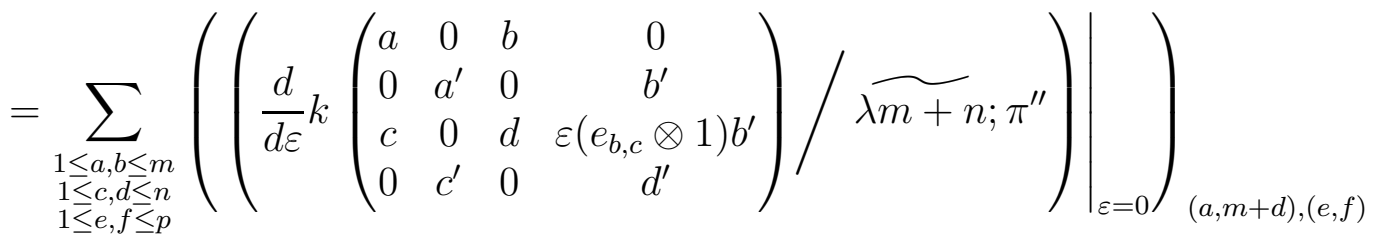


$e_{a b}^{(m)} \otimes e_{c d}^{(n)} \otimes e_{e f}^{(p)}$

Checking that $(\mathrm{id} \otimes \widetilde{\partial}) \circ \widetilde{\partial}=(\widetilde{\partial} \otimes \mathrm{id}) \circ \widetilde{\partial}$, after all these questions are put aside, boils down, like in the affine case to permuting the order in which we take two derivatives.

Lemma. If $\widetilde{h} \in A(\Omega)$ an $h=\widetilde{h}_{m+n+p}$, then

$$
(\mathrm{id} \otimes \widetilde{\partial})_{m, n, p} \widetilde{\partial}_{m, n+p} h=(\widetilde{\partial} \otimes \mathrm{id})_{m, n, p} \widetilde{\partial}_{m+n, p} h .
$$

Proof. Using the notations already introduced in this subsection, we have:

$\left((\mathrm{id} \otimes \widetilde{\partial})_{m, n, p} \circ \widetilde{\partial}_{m, n+p} h\right)\left(\pi ; \pi^{\prime} ; \pi^{\prime \prime}\right)_{(a, b)(c, d)(e, f)}$

$=\frac{d}{d \varepsilon_{1}}\left(\left.\frac{d}{d \varepsilon_{1}}\left(\left.\left.h\left(\left(\begin{array}{cccccc}a & 0 & 0 & b & 0 & 0 \\ 0 & a^{\prime} & 0 & 0 & b^{\prime} & 0 \\ 0 & 0 & a^{\prime \prime} & 0 & 0 & b^{\prime \prime} \\ c & 0 & 0 & d & \varepsilon_{1}\left(e_{b, c} \otimes 1\right) b^{\prime} & 0 \\ 0 & c^{\prime} & 0 & 0 & d^{\prime} & \varepsilon_{2}\left(e_{d, e} \otimes 1\right) b^{\prime \prime} \\ 0 & 0 & c^{\prime \prime} & 0 & 0 & d^{\prime \prime}\end{array}\right)\right)\right|_{(a, m+n+f)}\right|_{\varepsilon_{1}=0}\right)\right|_{\varepsilon_{2}=0}\right.$

Similarly we have:

$\left((\widetilde{\partial} \otimes \mathrm{id})_{m, n, p} \circ \widetilde{\partial}_{m+n, p} h\right)\left(\pi ; \pi^{\prime} ; \pi^{\prime \prime}\right)_{(a, b)(c, d)(e, f)}$

$=\frac{d}{d \varepsilon_{2}}\left(\left.\frac{d}{d \varepsilon_{1}}\left(\left.\left.h\left(\left(\begin{array}{cccccc}a & 0 & 0 & b & 0 & 0 \\ 0 & a^{\prime} & 0 & 0 & b^{\prime} & 0 \\ 0 & 0 & a^{\prime \prime} & 0 & 0 & b^{\prime \prime} \\ c & 0 & 0 & d & \varepsilon_{2}\left(e_{b, c} \otimes 1\right) b^{\prime} & 0 \\ 0 & c^{\prime} & 0 & 0 & d^{\prime} & \varepsilon_{1}\left(e_{d, e} \otimes 1\right) b^{\prime \prime} \\ 0 & 0 & c^{\prime \prime} & 0 & 0 & d^{\prime \prime}\end{array}\right)\right)\right|_{(a, m+n+f)}\right|_{\varepsilon_{1}=0}\right)\right|_{\varepsilon_{2}=0}\right.$.

Clearly the two quantities are equal (the only difference is that inside the $6 \times 6$ matrix we have replaced $\varepsilon_{1}$ by $\varepsilon_{2}$ and $\varepsilon_{2}$ by $\varepsilon_{1}$, so that the equality is just a permutability of partial derivatives).

\section{The RESOlVENT EQUATION AND THE DUALITY TRANSFORM}

6.1. We shall use the same framework as in sections 4 and 5, to carry out the computations which yield the functional equation for the Grassmannian resolvent $\left(\widetilde{\mathcal{R}}_{n}(\pi, B)(\cdot)\right)_{n \in \mathbb{N}}$ where

$$
\pi=\left(\begin{array}{ll}
a & b \\
c & d
\end{array}\right) / \widetilde{\lambda 1}=G r_{1}(E) .
$$


Let

$$
\sigma^{\prime}=\left(\begin{array}{cc}
\alpha^{\prime} & \beta^{\prime} \\
\gamma^{\prime} & \delta^{\prime}
\end{array}\right) / \widetilde{\lambda m} \in \widetilde{\rho}_{m}(\pi ; B)
$$

and let

$$
\sigma^{\prime \prime}=\left(\begin{array}{ll}
\alpha^{\prime \prime} & \beta^{\prime \prime} \\
\gamma^{\prime \prime} & \delta^{\prime \prime}
\end{array}\right) / \widetilde{\lambda n} \in \widetilde{\rho}_{n}(\pi ; B) .
$$

We then consider

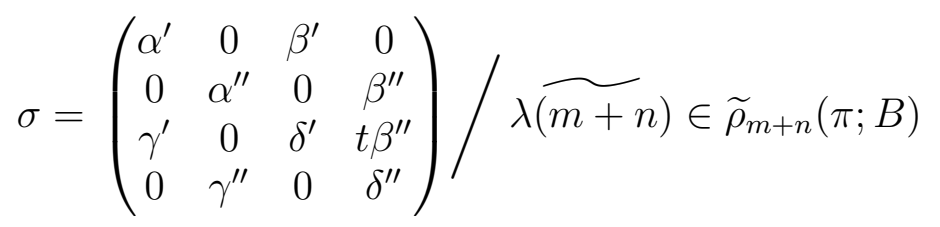

where $t \in \mathfrak{M}_{m, n}(\mathbb{C}) \subset \mathfrak{M}_{m, n}(B)$. To compute $\widetilde{\mathcal{R}}_{m+n}(\pi ; B)(\sigma)$ we must examine the matrix

$$
\Xi=\left(\begin{array}{ccc}
I_{m+n} \otimes b & \beta^{\prime} & 0 \\
& 0 & \beta^{\prime \prime} \\
I_{m+n} \otimes d & \delta^{\prime} & t \beta^{\prime \prime} \\
0 & \delta^{\prime \prime}
\end{array}\right)
$$

Permuting indices 2 and 3 in the above matrix, viewed as a $4 \times 4$ block-matrix, we get

$$
\begin{aligned}
& \Theta=\left(\begin{array}{cccc}
I_{m} \otimes b & \beta^{\prime} & 0 & 0 \\
I_{m} \otimes d & \delta^{\prime} & 0 & t \beta^{\prime \prime} \\
0 & 0 & I_{n} \otimes b & \beta^{\prime \prime} \\
0 & 0 & I_{n} \otimes d & \delta^{\prime \prime}
\end{array}\right) \\
& =\left(\begin{array}{cccc}
* & * & & \\
* & \zeta^{\prime} & & y \\
0 & 0 & * & * \\
0 & 0 & * & \zeta^{\prime \prime}
\end{array}\right)
\end{aligned}
$$

where

$$
\begin{aligned}
y & =-\left(\begin{array}{cc}
* & * \\
* & \zeta^{\prime}
\end{array}\right)\left(\begin{array}{cc}
0 & 0 \\
0 & t \beta^{\prime \prime}
\end{array}\right)\left(\begin{array}{cc}
* & * \\
* & \zeta^{\prime \prime}
\end{array}\right) \\
& =\left(\begin{array}{cc}
* & * \\
* & -\zeta^{\prime}+\beta^{\prime \prime} \zeta^{\prime \prime}
\end{array}\right)
\end{aligned}
$$

and $\beta^{\prime} \zeta^{\prime}=\widetilde{\mathcal{R}}_{m}(\pi ; B)\left(\sigma^{\prime}\right), \beta^{\prime \prime} \zeta^{\prime \prime}=\widetilde{\mathcal{R}}_{n}(\pi ; B)\left(\sigma^{\prime \prime}\right)$. This gives that

$$
\Theta=\left(\begin{array}{cccc}
* & * & * & * \\
* & \zeta^{\prime} & * & -\zeta^{\prime} t \beta^{\prime \prime} \zeta^{\prime \prime} \\
0 & 0 & * & * \\
0 & 0 & * & \zeta^{\prime \prime}
\end{array}\right)
$$


so that switching indices 2 and 3 we get

$$
\Xi=\left(\begin{array}{cccc}
* & * & * & * \\
0 & * & 0 & * \\
0 & * & \zeta^{\prime} & -\zeta^{\prime} t \beta^{\prime \prime} \zeta^{\prime \prime} \\
0 & * & 0 & \zeta^{\prime \prime}
\end{array}\right)
$$

The last formula implies

$$
\begin{aligned}
\widetilde{\mathcal{R}}_{m+n}(\pi ; B)(\sigma) & =\left(\begin{array}{cc}
\beta^{\prime} & 0 \\
0 & \beta^{\prime \prime}
\end{array}\right)\left(\begin{array}{cc}
\zeta^{\prime} & -\zeta^{\prime} t \beta^{\prime \prime} \zeta^{\prime \prime} \\
0 & \zeta^{\prime \prime}
\end{array}\right) \\
& =\left(\begin{array}{cc}
\widetilde{\mathcal{R}}_{m}(\pi ; B)\left(\sigma^{\prime}\right) & -\widetilde{\mathcal{R}}_{m}(\pi ; B)\left(\sigma^{\prime}\right) t R_{n}(\pi ; B)\left(\sigma^{\prime \prime}\right) \\
0 & \widetilde{\mathcal{R}}_{n}(\pi ; B)\left(\sigma^{\prime \prime}\right)
\end{array}\right) .
\end{aligned}
$$

Comparing this with the definition of $\widetilde{\partial}_{m, n} \widetilde{\mathcal{R}}(\pi ; B)$ we find that we have proved the following result.

\section{Lemma.}

$$
\left(\operatorname{id}_{E} \otimes \widetilde{\partial}_{m, n}\right) \widetilde{\mathcal{R}}_{m+n}(\pi ; B)\left(\sigma^{\prime} ; \sigma^{\prime \prime}\right)=-\widetilde{\mathcal{R}}_{m}(\pi ; B)\left(\sigma^{\prime}\right) \otimes_{E} \widetilde{\mathcal{R}}_{n}(\pi ; B)\left(\sigma^{\prime \prime}\right) .
$$

In the statement of the Lemma id ${ }_{E} \otimes \widetilde{\partial}_{m, n}$ refers to applying $\widetilde{\partial}$ to a $E$ valued fully matricial analytic function. The $\otimes_{E}$ among two matrices with entries in $E$ amounts to

$$
\left(\sum_{1 \leq i, j \leq m} c_{i j}^{\prime} \otimes e_{i j}^{(m)}\right) \otimes_{E}\left(\sum_{1 \leq k, l \leq n} c_{k l}^{\prime \prime} \otimes e_{k l}^{(n)}\right)=\sum_{i, j, k, l} c_{i j}^{\prime} c_{k l}^{\prime \prime} \otimes e_{i j}^{(m)} \otimes e_{k l}^{(n)} .
$$

We can write the resolvent equation also in a more compact form.

\section{Proposition.}

$$
\left(\operatorname{id}_{E} \otimes \widetilde{\partial}\right) \widetilde{\mathcal{R}}(\pi ; B)=-\widetilde{\mathcal{R}}(\pi ; B) \otimes_{E} \widetilde{\mathcal{R}}(\pi ; B) .
$$

6.2. Matrix entries of resolvents. An extension of the duality transform of [16], from the case of $Y \in E$ to the case of $\pi \in G r_{1}(E)$, includes in particular also the possibility of working with "unbounded operators $Y$ " represented by their graph and therefore the definition of the algebra $\mathcal{R} \mathcal{A}(Y ; B)$ in 9.1 of [16], which includes $Y$, must be replaced in our considerations here by the definition of an algebra where $Y$ does not appear. By $\mathcal{C R}(\pi ; B)$ we shall denote the set of matrix coefficients of $\left\{-\widetilde{\mathcal{R}}_{n}(\pi ; B)(\sigma) \mid n \in \mathbb{N}, \sigma \in \widetilde{\rho}_{n}(\pi ; B)\right\} . B y \mathcal{L} \mathcal{R}(\pi ; B)$ we shall denote the linear span of $\mathcal{C} \mathcal{R}(\pi ; B)$. 
Lemma. $\mathcal{C} \mathcal{R}(\pi ; B)$ is closed under multiplication. In particular $\mathcal{L} \mathcal{R}(\pi ; B)$ is a subalgebra of $E$.

Proof. The lemma is a consequence of the computations in 6.1. Indeed let $a, b$ be the $(i, j)$ and respectively the $(k, l)$ matrix-coefficient of $-\widetilde{\mathcal{R}}_{m}(\pi ; B)\left(\sigma^{\prime}\right)$ and $-\widetilde{\mathcal{R}}_{n}(\pi ; B)\left(\sigma^{\prime \prime}\right)$ and let $\sigma$ be defined like in 6.1 with $t=e_{j k}$. Then the computation of $-\widetilde{\mathcal{R}}_{m+n}(\pi ; B)(\sigma)$, we did, shows that its $(i, m+l)$-entry is exactly the $(i, l)$-entry of $\left(-\widetilde{\mathcal{R}}_{m}(\pi ; B)\left(\sigma^{\prime}\right)\right) e_{j k}\left(-\widetilde{\mathcal{R}}_{n}(\pi ; B)\left(\sigma^{\prime \prime}\right)\right)$ which is $a b$.

6.3. The duality transform. Let $E_{1}$ be the closure in $E$ of $\mathcal{L} \mathcal{R}(\pi ; B)$. We will define the duality transform associated with $\pi$ and $B$ on the topological dual $E_{1}^{d}$ of $E_{1}$. In general, the bialgebra structure is only "partially" defined on $E_{1}^{d}$ for analysis reasons, which cannot be dealt in this generality, we will therefore often look for formulations which avoid such problems or we will introduce extra assumptions (as we did in [16]). Some important instances when these assumptions are satisfied will be shown in $\S 12$.

If $\varphi \in E_{1}^{d}$, we define $\mathcal{U}(\varphi) \in \mathcal{A}(\widetilde{\rho}(\pi ; B))$ by $\mathcal{U}(\varphi)=\left(\mathcal{U}(\varphi)_{n}\right)_{n \in \mathbb{N}}$ where

$$
\mathcal{U}(\varphi)_{n}(\sigma)=\left(\operatorname{id}_{\mathfrak{M}_{n}} \otimes \varphi\right)\left(\widetilde{\mathcal{R}}_{n}(\pi ; B)(\sigma)\right)
$$

for $\sigma \in \widetilde{\rho}_{n}(\pi ; B)$. Since $\mathrm{id}_{\mathfrak{M}_{n}} \otimes \varphi$ is $\mathfrak{M}_{n}$-linear on $\mathfrak{M}_{n}(B)$ we infer that $\mathcal{U}(\varphi)$ is fully matricial since $\widetilde{\mathcal{R}}(\pi ; B)$ is fully matricial. The continuity assumption on $\varphi$ is necessary to obtain the analyticity of $\mathcal{U}(\varphi)$.

We also remark that $\mathcal{U}(\varphi)=0$ implies $\varphi=0$, that is $\mathcal{U}$ is injective. Indeed, $\mathcal{U}(\varphi)=0$ implies $\varphi \mid \mathcal{L} \mathcal{R}(\pi ; B)=0$ and $E_{1}$ is the closure of $\mathcal{L} \mathcal{R}(\pi ; B)$.

Up to now $\mathcal{L} \mathcal{R}(\pi ; B)$ is only an algebra so we have only a coalgebra structure on the dual (modulo technical problems). The behavior of $\mathcal{U}$ with respect to this comultiplication is recorded in the next proposition.

Proposition. If $\varphi \in E_{1}^{d}, \sigma^{\prime} \in \widetilde{\rho}_{m}(\pi ; B)$ and $\sigma^{\prime \prime} \in \widetilde{\rho}_{n}(\pi ; B)$, then we have

$$
\begin{aligned}
& \left(\operatorname{id}_{\mathfrak{M}_{m}} \otimes \operatorname{id}_{\mathfrak{M}_{n}} \otimes \varphi\right)\left(\widetilde{\mathcal{R}}_{m}(\pi ; B)\left(\sigma^{\prime}\right) \otimes_{E} \widetilde{\mathcal{R}}_{n}(\pi ; B)\left(\sigma^{\prime \prime}\right)\right) \\
& =-\widetilde{\partial}_{m, n}\left(\mathcal{U}(\varphi)_{m+n}\right)\left(\sigma^{\prime} ; \sigma^{\prime \prime}\right) .
\end{aligned}
$$

Proof. The proposition is exactly what we obtain from Lemma 6.1 when we apply $\mathrm{id}_{\mathfrak{M}_{m}} \otimes \mathrm{id}_{\mathfrak{M}_{n}} \otimes \varphi$ to the equality there. 
To justify our assertion that the above proposition shows that the behavior of $\mathcal{U}$ with respect to the comultiplication, note that the righthand side is the $(m, n)$-component of $-\widetilde{\partial} \mathcal{U}(\varphi)$, while the left-hand side corresponds to $(\mathcal{U} \otimes \mathcal{U})(\varphi \circ \mu)$ with $\mu$ denoting the multiplication on $\mathcal{L} \mathcal{R}(\pi ; B)$ (see also the proof of Lemma 6.2).

6.4. Further properties of the duality transform arise when there is an appropriate derivation-comultiplication on $\mathcal{L} \mathcal{R}(\pi ; B)$. To avoid questions such as the action of the derivation on elements of the Grassmannian, we will resort to a somewhat tautological (from the point of view of the duality transform) characterization of the derivation.

We will assume there is a derivation

$$
\partial_{\pi: B}: \mathcal{L R}(\pi ; B) \rightarrow \mathcal{L R}(\pi ; B) \otimes \mathcal{L R}(\pi ; B)
$$

such that

$$
\left(\operatorname{id}_{\mathfrak{M}_{n}} \otimes \partial_{\pi: B}\right) \widetilde{\mathcal{R}}_{n}(\pi ; B)(\sigma)=\widetilde{\mathcal{R}}_{n}(\pi ; B)(\sigma) \otimes_{\mathfrak{M}_{n}} \widetilde{\mathcal{R}}_{n}(\pi ; B)(\sigma)
$$

for all $n \in \mathbb{N}$ and $\sigma \in \widetilde{\rho}_{n}(\pi ; B)$.

For the universal unitary and hermitian Grassmannian elements this will be proved in $\S 12$.

Remark that in view of Lemma 6.2 the linear map $\partial_{\pi: B}$ is completely determined by the relation we assume. Thus the assumption means that this unique linear map exists and that it is a derivation. Note also that Lemma 6.2 similarly implies that $\partial_{\pi: B}$, if it exists, is coassociative.

Proposition. If $\varphi_{1}, \varphi_{2}, \varphi_{3} \in E_{1}^{d}$ are such that $\varphi_{1}(a)=\left(\varphi_{2} \otimes \varphi_{3}\right) \circ$ $\partial_{\pi: B}(a)$ if $a \in \mathcal{L} \mathcal{R}(\pi ; B)$, then we have

$$
\mathcal{U}\left(\varphi_{1}\right)=\mathcal{U}\left(\varphi_{2}\right) \mathcal{U}\left(\varphi_{3}\right)
$$

Proof. The proposition is almost obvious in view of the way we defined $\partial_{\pi: B}$.

Of course, as the reader probably already observed, the condition characterizing $\partial_{\pi: B}$ replace in the Grassmannian context the conditions $\partial B=0, \partial Y=1 \otimes 1$ we required in the affine case (see 9.2 in [16]), which corresponds to $\pi=\left(\begin{array}{ll}0 & 1 \\ 1 & Y\end{array}\right) / \widetilde{\lambda 1}$. 
6.5. The duality transform of traces. In this section we return to the context of 6.3 , that is we will not use the derivation-comultiplication of $\mathcal{L R}(\pi ; B)$. We will record here that Proposition 9.5 of [16] on transforms of traces in the affine case extends immediately to the Grassmannian setting.

Proposition. An element $\varphi \in E_{1}^{d}$ satisfies the trace-condition $\varphi\left(\left[E_{1}, E_{1}\right]\right)=$ 0 if and only if

$$
\widetilde{\partial}_{m, n}(\mathcal{U}(\varphi))_{m+n}\left(\sigma_{1} ; \sigma_{2}\right)=\varepsilon \circ \widetilde{\partial}_{n, m}(\mathcal{U}(\varphi))_{m+n}\left(\sigma_{2} ; \sigma_{1}\right)
$$

for all $\sigma_{1} \in \widetilde{\rho}_{m}(\pi ; B), \sigma_{2} \in \widetilde{\rho}_{n}(\pi ; B), m \in \mathbb{N}, n \in \mathbb{N}$. (Here $\varepsilon$ : $\mathfrak{M}_{m} \otimes \mathfrak{M}_{n} \rightarrow \mathfrak{M}_{n} \otimes \mathfrak{M}_{m}$ permutes the two factors.)

Proof. The trace condition $\varphi\left(\left[E_{1}, E_{1}\right]\right)$ is equivalent to

$$
\varphi\left(\left[\widetilde{\mathcal{R}}_{m}(\pi ; B)\left(\sigma_{1}\right)_{i, j}, \widetilde{\mathcal{R}}_{n}(\pi ; B)\left(\sigma_{2}\right)_{k, l}\right]\right)
$$

for all $\sigma_{1} \in \widetilde{\rho}_{m}(\pi ; B), \sigma_{2} \in \widetilde{\rho}_{n}(\pi ; B)$ and indices $i, j, k, l$. The last equality is then equivalent, by Proposition 6.3 to

$$
\widetilde{\partial}_{m, n}(\mathcal{U}(\varphi))_{m+n}\left(\sigma_{1} ; \sigma_{2}\right)=\varepsilon \circ \widetilde{\partial}_{n, m}(\mathcal{U}(\varphi))_{m+n}\left(\sigma_{2} ; \sigma_{1}\right) .
$$

\section{More on the FUlLy MATRICIAL AFFine SPACE}

Roughly, a large part of this section is about the analogue of polynomials in the context of fully matricial analytic functions on the fully matricial affine space. Besides providing a way to construct fully matricial analytic functions, this material will also underlie the series expansions in $\S 13$.

7.1. The polynomial sub-bialgebra $\mathcal{Z}\left(B^{d}\right)$ of $\mathcal{A}(\mathfrak{M}(B))$. Throughout 7.1 it will suffice to assume that $B$ is a complex Banach space and $1 \in B$ is a non-zero vector (used in the definition of $\partial$ ), there is no need for a multiplication on $B$ here.

The fully matricial affine space over $B$, that is the largest fully matricial $B$-set will be denoted $\mathfrak{M}(B)=\left(\mathfrak{M}_{n}(B)\right)_{n \in \mathbb{N}}$.

By $\mathbb{1} \in \mathcal{A}(\mathfrak{M}(B))$ we denote the unit element $\mathbb{1}=\left(I_{n} \otimes 1\right)_{n \in \mathbb{N}}$ (constant functions). If $\varphi \in B^{d}$ (the topological dual of $B$ ) we define $z(\varphi)=\left(z(\varphi)_{n}\right)_{n \in \mathbb{N}} \in \mathcal{A}(\mathfrak{M}(B))$ by

$$
z(\varphi)_{n}\left(\left(\begin{array}{ccc}
b_{11} & \ldots & b_{1 n} \\
\vdots & & \vdots \\
b_{n 1} & \ldots & b_{n n}
\end{array}\right)\right)=\left(\begin{array}{ccc}
\varphi\left(b_{11}\right) & \ldots & \varphi\left(b_{1 n}\right) \\
\vdots & & \vdots \\
\varphi\left(b_{n 1}\right) & \ldots & \varphi\left(b_{n n}\right)
\end{array}\right) \in \mathfrak{M}_{n}(\mathbb{C}) .
$$


Since $z(\varphi)_{n}$ is linear the definition of $\partial$ immediately gives

$$
\partial z(\varphi)=\varphi(1) \mathbb{1} \otimes \mathbb{1} .
$$

We shall denote by $\mathcal{Z}\left(B^{d}\right)$ the subalgebra of $\mathcal{A}(\mathfrak{M}(B))$ generated by $\mathbb{1}$ and $\left\{z(\varphi) \mid \varphi \in B^{d}\right\}$. It is easy to see that $\mathcal{Z}\left(B^{d}\right)$ is isomorphic to the tensor-algebra $\mathcal{T}\left(B^{d}\right)$ over the vector-space $B^{d}$. Indeed, if $\varphi_{1}, \ldots, \varphi_{n}$ are linearly independent in $B^{d}$ we can find $b_{1}, \ldots, b_{n} \in B$ so that $\varphi_{i}\left(b_{j}\right)=\delta_{i j}$. If $P \in \mathbb{C}\left\langle X_{1}, \ldots, X_{n}\right\rangle$ is a polynomial in the noncommuting indeterminates $X_{1}, \ldots, X_{n}$ so that $P \neq 0$, then there is $N \in \mathbb{N}$ so that we can find $N \times N$ matrices $A_{k} \in \mathfrak{M}_{N}(\mathbb{C}), 1 \leq k \leq n$ so that $P\left(A_{1}, \ldots, A_{n}\right) \neq 0$. Then $P\left(z\left(\varphi_{1}\right), \ldots, z\left(\varphi_{n}\right)\right)$ evaluated at $b_{1} A_{1}+\cdots+b_{n} A_{n} \in \mathfrak{M}_{N}(B)$ is precisely $P\left(A_{1}, \ldots, A_{n}\right) \in \mathfrak{M}_{N}(\mathbb{C})$. Thus $z\left(\varphi_{1}\right), \ldots, z\left(\varphi_{n}\right)$ are algebraically free. This suffices to guarantee that the natural unital homomorphism $\mathcal{T}\left(B^{d}\right) \rightarrow \mathcal{A}(\mathfrak{M}(B))$ defined by the linear map $B^{d} \ni \varphi \rightarrow z(\varphi) \in \mathcal{A}(\mathfrak{M}(B))$ is injective.

The fact that $\partial z(\varphi)=\varphi(1) \mathbb{1} \otimes \mathbb{1}$ implies that $\mathcal{Z}\left(B^{d}\right)$ is a subcoalgebra of $\mathcal{A}(\mathfrak{M}(B))$, that is

$$
\partial \mathcal{Z}\left(B^{d}\right) \subset \mathcal{Z}\left(B^{d}\right) \otimes \mathcal{Z}\left(B^{d}\right)
$$

Also the structure of $\partial$ on $\mathcal{Z}\left(B^{d}\right)$ is easy to identify. Let $1^{\perp}=\{\varphi \in$ $\left.B^{d} \mid \varphi(1)=0\right\}$ and choose some element $\theta \in B^{d}$ so that $\theta(1)=1$. Let then $\mathcal{Z}\left(1^{\perp}\right) \subset \mathcal{Z}\left(B^{d}\right)$ be the subalgebra of $\mathcal{Z}\left(B^{d}\right)$ generated by $\left\{z(\varphi) \mid \varphi \in 1^{\perp}\right\}$ and which is isomorphic to $\mathcal{T}\left(1^{\perp}\right)$. Then clearly $\mathcal{Z}\left(B^{d}\right)$ identifies with $\left(\mathcal{Z}\left(1^{\perp}\right)\right)\langle z(\theta)\rangle$ and $\mathcal{Z}\left(1^{\perp}\right)$ is in ker $\partial$ while $\partial z(\theta)=1 \otimes 1$. This means that the bialgebra $\mathcal{Z}\left(B^{d}\right)$ with the structure induced from $\mathcal{A}(\mathfrak{M}(B))$ is isomorphic to $\left.\left(\mathcal{T}\left(1^{\perp}\right)\right)\langle X\rangle, \partial_{X: \mathcal{T}\left(1^{\perp}\right)}\right)$. Note in particular that

$$
\operatorname{ker} \partial \cap \mathcal{Z}\left(B^{d}\right)=\mathcal{Z}\left(1^{\perp}\right)
$$

Moreover, if $B$ is a Banach space with a continuous conjugate-linear involution $b \rightarrow b^{*}$, then $\mathcal{Z}\left(B^{d}\right)$ has an involution $(z(\varphi))^{*}=z\left(\varphi^{*}\right)$ where $\varphi^{*}(b)=\overline{\varphi\left(b^{*}\right)}$.

Also, at the end of 8.2 we will point out in a Remark an additional feature of $\mathcal{Z}\left(B^{d}\right)$.

7.2. Decomposable and reducible points in $\mathfrak{M}(B)$. Like in 7.1, also in 7.2 , we will only require that $B$ be a Banach space.

In view of the similarity and direct sum requirements for "fully matricial" objects, we are led to look at properties of points connected with these requirements.

Definition. An element $\beta \in \mathfrak{M}_{n}(B)$ is decomposable if there are $\beta^{\prime} \in$ $\mathfrak{M}_{p}(B), \beta^{\prime \prime} \in \mathfrak{M}_{q}(B)$ and $S \in G L(n ; \mathbb{C})$, so that $n=p+q, p>0, q>0$ 
and $S \beta S^{-1}=\beta^{\prime} \oplus \beta^{\prime \prime}$. An element $\beta \in \mathfrak{M}_{n}(B)$ is reducible if there are $\beta^{\prime} \in \mathfrak{M}_{p}(B), \beta^{\prime \prime} \in \mathfrak{M}_{q}(B), \gamma \in \mathfrak{M}_{p, q}(B)$ and $S \in G L(n ; \mathbb{C})$ so that

$$
S \beta S^{-1}=\left(\begin{array}{cc}
\beta^{\prime} & \gamma \\
0 & \beta^{\prime \prime}
\end{array}\right)
$$

and $p>0, q>0$. An element $\beta \in \mathfrak{M}_{n}(B)$ is approximately decomposable (resp. reducible) if it is in the closure of the decomposable (resp. reducible) elements. Elements which are not decomposable (reducible, approximately decomposable, approximately reducible) will be called indecomposable (resp. irreducible, strongly indecomposable, strongly irreducible).

7.3. To conclude this section of remarks about the fully matricial affine space, we should point out that there is a fully matricial action of the additive group $B$ on $\mathfrak{M}(B)$. For each $b \in B$ there are fully matricial maps $T(b)=\left(T(b)_{n}\right)_{n \in \mathbb{N}}: \mathfrak{M}(B) \rightarrow \mathfrak{M}(B)$ where $T(b)_{n}(\beta)=\beta+b \otimes I_{n}$ which give an action of $B$ on $\mathfrak{M}(B)$.

In case $B$ is a Banach algebra, there is also a multiplication action given by fully matricial maps $L(b)=\left(L(b)_{n}\right)_{n \in \mathbb{N}}, R(b)=\left(R(b)_{n}\right)_{n \in \mathbb{N}}$ so that $L(b) \beta=\left(b \otimes I_{n}\right) \beta$ and $R(b) \beta=\beta\left(b \otimes I_{n}\right)$.

Note also that even if $B$ is only a Banach space there is a multiplicative action of $\mathbb{C}$ on $\mathfrak{M}(B)$.

\section{More on the FUlly matricial $B$-Grassmannian AND ON $\widetilde{\partial}$}

In this section we present further properties of the fully matricial $B$-Grassmannian $\operatorname{Gr}(B)=\left(G r_{n}(B)\right)_{n \in \mathbb{N}}$. This includes the action by fully matricial automorphisms of $G L(2 ; B)$ on $G r(B)$ and the existence of a coderivation $\Lambda$ such that $\Lambda$ - id plays the role of a grading of the bialgebras $\mathcal{A}(\Omega)$. We also discuss the properties of $\Lambda$ in connection with the duality transform.

8.1. The $G L(2 ; B)$ action on $\operatorname{Gr}(B)$. We recall that in 3.2 we defined $g \pi$ if $g \in G L_{2}\left(\mathfrak{M}_{n}(B)\right)$ and $\pi \in G r_{n}(B)$ and $s \cdot \pi$ if $s \in G L(n ; \mathbb{C})$. An element $h=\left(\begin{array}{ll}b_{11} & b_{12} \\ b_{21} & b_{22}\end{array}\right) \in G L(2 ; B)$ gives rise to elements $h_{n} \in$ $G L_{2}\left(\mathfrak{M}_{n}(B)\right)$ where $h_{n}=\left(\begin{array}{ll}I_{n} \otimes b_{11} & I_{n} \otimes b_{12} \\ I_{n} \otimes b_{21} & I_{n} \otimes b_{22}\end{array}\right)$. We define $C(h)$ : $G r(B) \rightarrow G r(B)$ by mapping $\pi_{n} \in G r_{n}(B)$ to $h_{n} \pi_{n}$. It is easy to check that $h_{m+n}\left(\pi_{m} \oplus \pi_{n}\right)=\left(h_{m} \pi_{m}\right) \oplus\left(h_{n} \pi_{n}\right)$ and that $h_{n}\left(s \cdot \pi_{n}\right)=s \cdot\left(h_{n} \pi_{n}\right)$ if $s \in G L(n ; \mathbb{C})$. This establishes that $C(h)$ is a fully matricial map of $\operatorname{Gr}(B)$ into $\operatorname{Gr}(B)$. It is immediate from the definition that $C(\cdot)$ is an action of $G L(2 ; B)$ by fully matricial automorphisms of $G r(B)$. 
It is easily seen that $C(h)$ preserves transversality in each $G r_{n}(B)$.

Clearly, when $B=\mathbb{C}$ the $G L(2 ; \mathbb{C})$-action on $G r_{1}(\mathbb{C})$ is the usual action on the Riemann sphere by fractional linear transformations.

8.2. The coderivation $\Lambda$. Let $f=\left(f_{n}\right)_{n \in \mathbb{N}} \in \mathcal{A}(\Omega)$, where $\Omega=$ $\left(\Omega_{n}\right)_{n \in \mathbb{N}}$ is a fully matricial open $B$-set of the Grassmannian. We define $\Lambda f=\left(\Lambda_{n} f_{n}\right)_{n \in \mathbb{N}} \in \mathcal{A}(\Omega)$, by

$$
\Lambda f=\left.\frac{d}{d t}\left(e^{t} f \circ C\left(\left(\begin{array}{cc}
1 & 0 \\
0 & e^{t}
\end{array}\right)\right)\right)\right|_{t=0}
$$

which, componentwise, amounts to

$$
\left(\Lambda_{n} f_{n}\right)\left(\pi_{n}\right)=\left.\frac{d}{d t}\left(e^{t} f\left(\left(\begin{array}{cc}
1 & 0 \\
0 & e^{t}
\end{array}\right)_{n} \pi_{n}\right)\right)\right|_{t=0} .
$$

Since

$$
\Lambda f-f=\left.\frac{d}{d t}\left(f \circ C\left(\left(\begin{array}{cc}
1 & 0 \\
0 & e^{t}
\end{array}\right)\right)\right)\right|_{t=0}
$$

it follows that $\Lambda-\mathrm{id}$ is a derivation of $\mathcal{A}(\Omega)$.

To prove that $\Lambda$ is a coderivation amounts to proving that

$$
\widetilde{\partial} \circ \Lambda=(\Lambda \otimes \mathrm{id}+\mathrm{id} \otimes \Lambda) \circ \widetilde{\partial} .
$$

This will be a consequence of the following lemma.

Lemma. We have

$\widetilde{\partial}\left(f \circ C\left(\left(\begin{array}{cc}1 & 0 \\ 0 & e^{t}\end{array}\right)\right)\right)=e^{t}(\widetilde{\partial} f) \circ\left(C\left(\left(\begin{array}{cc}1 & 0 \\ 0 & e^{t}\end{array}\right)\right) \times C\left(\left(\begin{array}{cc}1 & 0 \\ 0 & e^{t}\end{array}\right)\right)\right)$.

Proof. Let $\pi_{m}=\left(\begin{array}{ll}a_{1} & b_{1} \\ c_{1} & d_{1}\end{array}\right) / \widetilde{\lambda m}, \pi_{n}=\left(\begin{array}{ll}a_{2} & b_{2} \\ c_{2} & d_{2}\end{array}\right) / \widetilde{\lambda n}$ and let $T$ and $T^{\prime}$ be defined by

$$
\begin{aligned}
T & =\alpha_{m, n}\left(\widetilde{\partial}_{m, n}\left(f \circ C\left(\left(\begin{array}{cc}
1 & 0 \\
0 & e^{t}
\end{array}\right)\right)\right)\left(\pi_{m}, \pi_{n}\right)\right) \\
T^{\prime} & =\alpha_{m, n}\left(\widetilde{\partial}_{m, n}(f)\left(\left(\begin{array}{cc}
1 & 0 \\
0 & e^{t}
\end{array}\right)_{m} \pi_{m},\left(\begin{array}{cc}
1 & 0 \\
0 & e^{t}
\end{array}\right) \pi_{n}\right)\right) .
\end{aligned}
$$

Since $\alpha_{m, n}$ is an isomorphism, it will suffice to prove that $T(s)=T^{\prime}\left(e^{t} s\right)$ for all $s \in \mathfrak{M}_{m, n}(\mathbb{C})$. Indeed, we have:

$$
f_{m+n}\left(\left(\begin{array}{cc}
1 & 0 \\
0 & e^{t}
\end{array}\right)_{m+n}\left(\begin{array}{cccc}
a_{1} & 0 & b_{1} & 0 \\
0 & a_{2} & 0 & b_{2} \\
c_{1} & 0 & d_{1} & s b_{2} \\
0 & c_{2} & 0 & d_{2}
\end{array}\right) / \widetilde{\lambda m+n}\right)
$$




$$
\begin{aligned}
& =f_{m+n}\left(\left(\begin{array}{cccc}
a_{1} & 0 & b_{1} & 0 \\
0 & a_{2} & 0 & b_{2} \\
e^{t} c_{1} & 0 & e^{t} d_{1} & e^{t} s b_{2} \\
0 & e^{t} c_{2} & 0 & e^{t} d_{2}
\end{array}\right) / \widetilde{\lambda m+n}\right. \\
& =\left(\begin{array}{cc}
f_{m}\left(\left(\begin{array}{cc}
1 & 0 \\
0 & e^{t}
\end{array}\right)_{m}\left(\begin{array}{cc}
a_{1} & b_{1} \\
c_{1} & d_{1}
\end{array}\right) / \widetilde{\lambda m}\right. \\
0 & \left.f_{n}\left(\begin{array}{cc}
1 & 0 \\
0 & e^{t}
\end{array}\right)_{n}\left(\begin{array}{ll}
a_{2} & b_{2} \\
c_{2} & d_{2}
\end{array}\right) / \widetilde{\lambda n}\right)
\end{array}\right) \\
& =\left(\begin{array}{cc}
f_{m}\left(\left(\begin{array}{cc}
a_{1} & b_{1} \\
e^{t} c_{1} & e^{t} d_{2}
\end{array}\right) / \widetilde{\lambda m}\right) & T^{\prime}\left(e^{t} s\right) \\
0 & \left.f_{n}\left(\begin{array}{cc}
a_{2} & b_{2} \\
e^{t} c_{2} & e^{t} d_{2}
\end{array}\right) / \widetilde{\lambda n}\right)
\end{array}\right),
\end{aligned}
$$

which implies $T(s)=T^{\prime}\left(e^{t} s\right)$.

To conclude the proof of the fact that $\Lambda$ is a coderivation it will suffice to remark that taking the derivative $\frac{d}{d t}$ at $t=0$ of the equality in the preceding lemma gives

$$
\widetilde{\partial}(\Lambda f-f)=\widetilde{\partial} f+((\Lambda-\mathrm{id}) \otimes \mathrm{id}+\mathrm{id} \otimes(\Lambda-\mathrm{id})) \widetilde{\partial} f
$$

which immediately implies

$$
\widetilde{\partial} \Lambda f=(\Lambda \otimes \mathrm{id}+\mathrm{id} \otimes \Lambda) \widetilde{\partial} f .
$$

Proposition. $\Lambda$ - id is a derivation of $\mathcal{A}(\Omega)$ and $\Lambda$ is also a coderivation, that is $\widetilde{\partial} \circ \Lambda=(\Lambda \otimes \mathrm{id}+\mathrm{id} \otimes \Lambda) \circ \widetilde{\partial}$.

8.3. The derivation $D$ of $\mathcal{L} \mathcal{R}(\pi ; B)$. In the next section we will show that the coderivation $\Lambda$ discussed in the previous section is natural from the point of view of the duality transform. This will involve describing what the natural coderivation $L$ on $\mathcal{L} \mathcal{R}(\pi ; B)$ should be so that for the duality described in Theorem 5.3 of [15], the dual coderivation corresponds under the duality transform to $\Lambda$. Since in 6.4 we assumed the existence of a derivation-comultiplication $\partial_{\pi: B}$ on $\mathcal{L} \mathcal{R}(\pi ; B)$, we will handle $L$ similarly based on an additional assumption.

Remark. In the affine case of $\mathfrak{M}(B)$, we have $\Lambda \mathcal{Z}\left(B^{d}\right) \subset \mathcal{Z}\left(B^{d}\right)$ and

$$
\Lambda\left(z\left(\varphi_{1}\right) \ldots z\left(\varphi_{n}\right)\right)=(n+1) z\left(\varphi_{1}\right) \ldots z\left(\varphi_{n}\right) .
$$

Like in 6.2 we let $\pi \in G r_{1}(E)$ and we consider $\mathcal{L} \mathcal{R}(\pi ; B)$. The assumption about $L$ is roughly that on $\mathcal{L} \mathcal{R}(\pi ; B)$ there is a linear map 
$D$ corresponding to the infinitesimal deformation of $\pi$ into $\left(\begin{array}{cc}1 & 0 \\ 0 & e^{t}\end{array}\right)_{1} \pi$ with $t \rightarrow 0$. We will show that $D$ must then be a derivation of $\mathcal{L} \mathcal{R}(\pi ; B)$ with values in itself. (In case $\pi$ is the graph of an element $Y \in E$, the deformation is $Y \rightarrow e^{t} Y$ with $t \rightarrow 0$.)

More precisely our assumption can be formulated as follows: $W e$ assume there is a linear map $D: \mathcal{L R}(\pi ; B) \rightarrow E$ so that

$$
\left(\operatorname{id}_{\mathfrak{M}_{n}} \otimes D\right) \widetilde{\mathcal{R}}_{n}(\pi ; B)(\sigma)=\left.\frac{d}{d t} \widetilde{\mathcal{R}}_{n}\left(\left(\begin{array}{cc}
1 & 0 \\
0 & e^{t}
\end{array}\right)_{1} \pi ; B\right)(\sigma)\right|_{t=0}
$$

for all $\sigma \in \widetilde{\rho}_{n}(\pi ; B), n \in \mathbb{N}$. There is a simple identity which we will use to show that $D$ takes values in $\mathcal{L} \mathcal{R}(\pi ; B)$.

Lemma. We have $\sigma \in \widetilde{\rho}_{n}\left(\left(\begin{array}{cc}1 & 0 \\ 0 & e^{t}\end{array}\right)_{1} \pi ; B\right)$ iff $\left(\begin{array}{cc}1 & 0 \\ 0 & e^{-t}\end{array}\right)_{n} \sigma \in \widetilde{\rho}_{n}(\pi ; B)$. Moreover, then

$$
\widetilde{\mathcal{R}}_{n}\left(\left(\begin{array}{cc}
1 & 0 \\
0 & e^{t}
\end{array}\right)_{1} \pi ; B\right)(\sigma)=e^{-t} \widetilde{\mathcal{R}}_{n}(\pi ; B)\left(\left(\begin{array}{cc}
1 & 0 \\
0 & e^{-t}
\end{array}\right)_{n} \sigma\right) .
$$

Proof. Let $\pi=\left(\begin{array}{ll}a & b \\ c & d\end{array}\right) / \widetilde{\lambda 1}, \sigma=\left(\begin{array}{ll}\alpha & \beta \\ \gamma & \delta\end{array}\right) / \widetilde{\lambda n}$ and let also $\pi^{\prime}=$ $\left(\begin{array}{ll}I_{n} \otimes a & I_{n} \otimes b \\ I_{n} \otimes c & I_{n} \otimes d\end{array}\right) / \widetilde{\lambda n}$ and $a^{\prime}=I_{n} \otimes a, b^{\prime}=I_{n} \otimes b, c^{\prime}=I_{n} \otimes c, d^{\prime}=$ $I_{n} \otimes d$. Then $\sigma \in \widetilde{\rho}_{n}\left(\left(\begin{array}{cc}1 & 0 \\ 0 & e^{t}\end{array}\right)_{1} \pi ; B\right)$ means $\left(\begin{array}{cc}b^{\prime} & \beta \\ e^{t} d^{\prime} & \delta\end{array}\right)$ is invertible and this is obviously equivalent to $\left(\begin{array}{cc}b^{\prime} & \beta \\ d^{\prime} & e^{-t} \delta\end{array}\right)$ being invertible, which is that $\left(\begin{array}{cc}1 & 0 \\ 0 & e^{-t}\end{array}\right)_{n} \sigma \in \widetilde{\rho}_{n}(\pi ; B)$.

Moreover if $\left(\begin{array}{cc}b^{\prime} & \beta \\ e^{t} d^{\prime} & \delta\end{array}\right)^{-1}=\left(\begin{array}{cc}* & * \\ * & \zeta\end{array}\right)$ and $\left(\begin{array}{cc}b^{\prime} & \beta \\ d^{\prime} & e^{t} \delta\end{array}\right)^{-1}=\left(\begin{array}{cc}* & * \\ * & \xi\end{array}\right)$, then $\zeta=e^{-t} \xi$. The last part of the lemma follows from the two Grassmannian resolvents being equal to $\beta \zeta$ and $\beta \xi$ respectively.

With the notations used in the proof of the lemma, to show that $D(\mathcal{L} \mathcal{R}(\pi ; B) \subset \mathcal{L} \mathcal{R}(\pi ; B))$ we must prove in view of the definition of $D$ that the entries of $\left.\frac{d}{d t}(\beta \zeta(t))\right|_{t=0}$ are in $\mathcal{L} \mathcal{R}(\pi ; B)$ or equivalently the entries of $\left.\frac{d}{d t}\left(e^{-t} \beta \xi(t)\right)\right|_{t=0}$. Since $\beta \xi(0)=\beta \zeta(0)$ is a resolvent, its entries 
are in $\mathcal{L} \mathcal{R}(\pi ; B)$, so we are left with showing $\left.\beta\left(\frac{d}{d t} \xi(t)\right)\right|_{t=0}$ has entries in $\mathcal{L} \mathcal{R}(\pi ; B)$. We have

$$
\left(\begin{array}{cc}
* & * \\
* & \frac{d}{d t} \xi(t)
\end{array}\right)=\frac{d}{d t}\left(\begin{array}{cc}
b^{\prime} & \beta \\
d^{\prime} & e^{-t} \delta
\end{array}\right)^{-1}=\left(\begin{array}{cc}
* & * \\
* & \xi
\end{array}\right)\left(\begin{array}{cc}
0 & 0 \\
0 & e^{-t} \delta
\end{array}\right)\left(\begin{array}{cc}
* & * \\
* & \xi
\end{array}\right) .
$$

Hence we infer that

$$
\left.\left(\frac{d}{d t} \xi(t)\right)\right|_{t=0}=\xi(0) \delta \xi(0)
$$

and we must show that $\beta \xi(0) \delta \xi(0)$ has entries in $\mathcal{L} \mathcal{R}(\pi ; B)$. It is easily seen that the $(2,4)$-block entry of the $4 \times 4$ block matrix

$$
\Gamma^{-1}=\left(\begin{array}{cccc}
b^{\prime} & \beta & 0 & 0 \\
d^{\prime} & \delta & 0 & \delta \\
0 & 0 & b^{\prime} & \beta \\
0 & 0 & d^{\prime} & \delta
\end{array}\right)^{-1}
$$

is precisely $\xi(0) \delta \xi(0)$. On the other hand if $S$ is the permutation matrix

$$
S=\left(\begin{array}{cccc}
I_{n} & 0 & 0 & 0 \\
0 & 0 & I_{n} & 0 \\
0 & I_{n} & 0 & 0 \\
0 & 0 & 0 & I_{n}
\end{array}\right)
$$

we see that

$$
S \Gamma^{-1} S^{-1}=\left(\begin{array}{cccc}
b^{\prime} & 0 & \beta & 0 \\
0 & b^{\prime} & 0 & \beta \\
d^{\prime} & 0 & \delta & \delta \\
0 & d^{\prime} & 0 & \delta
\end{array}\right)^{-1}=\left(\begin{array}{cc}
* & * \\
* & Z
\end{array}\right)
$$

where $\left(\begin{array}{cc}\beta & 0 \\ 0 & \beta\end{array}\right) Z$ is an $\widetilde{\mathcal{R}}_{2 n}(\pi ; B)(\mu)$ for some $\mu=\widetilde{\rho}_{2 n}(\pi ; B)$. Hence the entries of $\left(\begin{array}{ll}\beta & 0 \\ 0 & \beta\end{array}\right) Z$ are in $\mathcal{L} \mathcal{R}(\pi ; B)$. Returning to $\Gamma^{-1}$ we see that the $(2,4)$-block entry of $\Gamma^{-1}$ coincides with the $(3,4)$-block entry of $S \Gamma^{-1} S^{-1}$ which is the $(1,2)$-block entry of $Z$ (the blocks are $n \times n$ ). This concludes the proof that $D$ maps $\mathcal{L} \mathcal{R}(\pi ; B)$ into itself.

To prove that $D$ is a derivation we return to the proof of Lemma 6.2 where we showed $\mathcal{C} \mathcal{R}(\pi ; B)$ is closed under multiplication. With the notation of Lemma 6.2 we have

$$
-\left(\widetilde{\mathcal{R}}_{m+n}(\pi ; B)(\sigma)\right)_{i, m+l}=\left(\widetilde{\mathcal{R}}_{m}(\pi ; B)\left(\sigma^{\prime}\right)\right)_{i j}\left(\widetilde{\mathcal{R}}_{n}(\pi ; B)\left(\sigma^{\prime \prime}\right)\right)_{k l}
$$

where $\sigma^{\prime}, \sigma^{\prime \prime},(i, j),(k, l)$ were given. Then the definition of $D$ applied to the above equality shows that $D$ is a derivation. Concluding we have proved 
Proposition. Under our assumptions $D$ is a derivation of $\mathcal{L} \mathcal{R}(\pi ; B)$ into itself.

8.4. The coderivation $L$ of $\mathcal{L} \mathcal{R}(\pi ; B)$. In this section we assume the existence of $\partial_{\pi: B}$ with the properties outlined in 6.4 and we also assume the existence of the linear map D like in 8.3 and which implies that $D$ is a derivation of $\mathcal{L} \mathcal{R}(\pi ; B)$. In addition, we will assume that $\partial_{\pi: B}$ is closed as an operator on $\mathcal{L} \mathcal{R}(\pi ; B)$ endowed with the norm from E.

We define

$$
L: D+\mathrm{id}: \mathcal{L} \mathcal{R}(\pi ; B) \rightarrow \mathcal{L} \mathcal{R}(\pi ; B) .
$$

Clearly $L-$ id is a derivation of $\mathcal{L} \mathcal{R}(\pi ; B)$.

Lemma. The map $L$ is a coderivation of $\left(\mathcal{L R}(\pi ; B), \partial_{\pi: B}\right)$, that is

$$
\partial_{\pi: B} \circ L=(\mathrm{id} \otimes L+L \otimes \mathrm{id}) \circ \partial_{\pi: B} .
$$

Proof. Since $\mathcal{L} \mathcal{R}(\pi ; B)$ is the linear span of $\mathcal{C} \mathcal{R}(\pi ; B)$ it suffices to check that the equality to be proved holds for the entries of $\widetilde{\mathcal{R}}_{n}(\pi ; B)(\sigma)$. In view of the definitions of $\partial_{\pi: B}$ and $L$ this boils down to showing that

$$
\begin{aligned}
& \left.\left(\operatorname{id}_{\mathfrak{M}_{n}} \otimes \partial_{\pi: B}\right)\left(\frac{d}{d t} \widetilde{\mathcal{R}}_{n}(\pi ; B)\left(\left(\begin{array}{cc}
1 & 0 \\
0 & e^{-t}
\end{array}\right)_{n} \sigma\right)\right)\right|_{t=0} \\
& =\left.\frac{d}{d t} \widetilde{\mathcal{R}}_{n}(\pi ; B)\left(\left(\begin{array}{cc}
1 & 0 \\
0 & e^{-t}
\end{array}\right)_{n} \sigma\right)\right|_{t=0} \otimes_{\mathfrak{M}_{n}} \widetilde{\mathcal{R}}_{n}(\pi ; B)(\sigma) \\
& +\left.\widetilde{\mathcal{R}}_{n}(\pi ; B)(\sigma) \otimes_{\mathfrak{M}_{n}} \frac{d}{d t} \widetilde{\mathcal{R}}_{n}(\pi ; B)\left(\left(\begin{array}{cc}
1 & 0 \\
0 & e^{-t}
\end{array}\right)_{n} \sigma\right)\right|_{t=0} .
\end{aligned}
$$

It is immediate that the right-hand side equals

$$
\begin{aligned}
& \left.\frac{d}{d t}\left(\widetilde{\mathcal{R}}_{n}(\pi ; B)\left(\left(\begin{array}{cc}
1 & 0 \\
0 & e^{-t}
\end{array}\right)_{n} \sigma\right) \otimes_{\mathfrak{M}_{n}} \widetilde{\mathcal{R}}_{n}(\pi ; B)\left(\left(\begin{array}{cc}
1 & 0 \\
0 & e^{-t}
\end{array}\right)_{n} \sigma\right)\right)\right|_{t=0} \\
& =\left.\frac{d}{d t}\left(\operatorname{id}_{\mathfrak{M}_{n}} \otimes \partial_{\pi: B}\right) \widetilde{\mathcal{R}}_{n}(\pi ; B)\left(\left(\begin{array}{cc}
1 & 0 \\
0 & e^{-t}
\end{array}\right)_{n} \sigma\right)\right|_{t=0} .
\end{aligned}
$$

Thus the equality to be proved reduces to showing that

$$
\begin{aligned}
& \left.\frac{d}{d t}\left(\operatorname{id}_{\mathfrak{M}_{n}} \otimes \partial_{\pi: B}\right) \widetilde{\mathcal{R}}_{n}(\pi ; B)\left(\left(\begin{array}{cc}
1 & 0 \\
0 & e^{-t}
\end{array}\right)_{n} \sigma\right)\right|_{t=0} \\
& =\left(\operatorname{id}_{\mathfrak{M}_{n}} \otimes \partial_{\pi: B}\right)\left(\left.\frac{d}{d t} \widetilde{\mathcal{R}}_{n}(\pi ; B)\left(\left(\begin{array}{cc}
1 & 0 \\
0 & e^{-t}
\end{array}\right)_{n} \sigma\right)\right|_{t=0}\right) .
\end{aligned}
$$

Clearly, the last equality is a consequence of the assumption that $\partial_{\pi: B}$ is closed. 
8.5. The coderivations $L$ and $\Lambda$ and the duality transform. In this section the same assumptions as in 8.4 will hold throughout.

Let $E_{1}$ be the closure of $\mathcal{L} \mathcal{R}(\pi ; B)$ in $E$ and let $\varphi \in E_{1}^{d}$ so that $\varphi$ is in the domain of $L^{d}$, that is $\varphi \circ L$ defined on $\mathcal{L} \mathcal{R}(\pi ; B)$ is bounded (extends to an element of $E_{1}^{d}$ ).

Recall that the $n$-th component of the duality transform is defined by

$$
\mathcal{U}(\varphi)_{n}(\sigma)=\left(\operatorname{id}_{\mathfrak{M}_{n}} \otimes \varphi\right)\left(\widetilde{\mathcal{R}}_{n}(\pi ; B)(\sigma)\right)
$$

We have

$$
\begin{aligned}
\mathcal{U}\left(L^{d} \varphi\right)_{n}(\sigma) & =\left(\operatorname{id}_{\mathfrak{M}_{n}} \otimes \varphi\right)\left(\operatorname{id}_{\mathfrak{M}_{n}} \otimes L\right)\left(\widetilde{\mathcal{R}}_{n}(\pi ; B)(\sigma)\right) \\
& =\left.\left(\operatorname{id}_{\mathfrak{M}_{n}} \otimes \varphi\right) \frac{d}{d t} \widetilde{\mathcal{R}}_{n}(\pi ; B)\left(\left(\begin{array}{cc}
1 & 0 \\
0 & e^{-t}
\end{array}\right)_{n}(\sigma)\right)\right|_{t=0} \\
& =\left.\frac{d}{d t}\left(\operatorname{id}_{\mathfrak{M}_{n}} \otimes \varphi\right)\left(\widetilde{\mathcal{R}}_{n}(\pi ; B)\left(\left(\begin{array}{cc}
1 & 0 \\
0 & e^{-t}
\end{array}\right)_{n}(\sigma)\right)\right)\right|_{t=0} \\
& =-\left.\frac{d}{d t} \mathcal{U}(\varphi)_{n}\left(\left(\begin{array}{cc}
1 & 0 \\
0 & e^{t}
\end{array}\right)_{n} \sigma\right)\right|_{t=0} \\
& =-\left((\Lambda-\mathrm{id}) \mathcal{U}(\varphi)_{n}\right)(\sigma)=(\mathrm{id}-\Lambda) \mathcal{U}(\varphi)_{n}(\sigma) .
\end{aligned}
$$

Thus we have proved the following proposition.

Proposition. We have $\mathcal{U}\left(L^{d} \varphi\right)=(\mathrm{id}-\Lambda) \mathcal{U}(\varphi)$.

Note that the way the coderivation should be transformed under duality given in [15, Thm. 5.3] is in agreement with the above Proposition.

\section{The Grassmannian involution}

Throughout this section $B$ will be a unital Banach algebra with involution. We will discuss the corresponding involutions on $\operatorname{Gr}(B)$ and bialgebras $\mathcal{A}(\Omega)$, and the properties of the duality transform related to the involutions.

9.1. The involution on $\operatorname{Gr}(B)$. On the affine fully matricial space the involution amounts simply to the conjugate-linear antiautomorphism $T \rightarrow T^{*}$ on $\mathfrak{M}_{n}(B), n \in \mathbb{N}$. The extension to an antiholomorphic automorphism of the fully matricial $B$-Grassmannian has some additional technical points.

We will first define the orthogonal $\pi^{1}$ of $\pi \in G r_{n}(B)$ and then we shall define $\pi^{*}=\left(\begin{array}{cc}0 & 1 \\ -1 & 0\end{array}\right)_{n} \pi^{\perp}$. 
If $\pi=\left(\begin{array}{ll}a & b \\ c & d\end{array}\right) / \widetilde{\lambda n}$ we define $\pi^{\perp}=\left(\begin{array}{ll}z^{*} & x^{*} \\ t^{*} & y^{*}\end{array}\right) / \widetilde{\lambda n}$ where $\left(\begin{array}{ll}x & y \\ z & t\end{array}\right)=$ $\left(\begin{array}{ll}a & b \\ c & d\end{array}\right)^{-1}$. To check that $\pi^{\perp}$ is well-defined we begin with a simple algebraic lemma.

Lemma. Suppose that $\left(\begin{array}{ll}a & b \\ c & d\end{array}\right)^{-1}=\left(\begin{array}{ll}x & y \\ z & t\end{array}\right)$ and $\left(\begin{array}{ll}a^{\prime} & b \\ c^{\prime} & d\end{array}\right)^{-1}=\left(\begin{array}{ll}x^{\prime} & y^{\prime} \\ z^{\prime} & t^{\prime}\end{array}\right)$. Then $\left(\begin{array}{cc}x & y \\ z^{\prime} & t^{\prime}\end{array}\right)$ is invertible and there is $w$ invertible so that $w x=x^{\prime}$, $w y=y^{\prime}$.

Proof. Since

$$
\left(\begin{array}{ll}
x & y \\
z^{\prime} & t^{\prime}
\end{array}\right)\left(\begin{array}{ll}
a & b \\
c & d
\end{array}\right)=\left(\begin{array}{ll}
1 & 0 \\
* & 1
\end{array}\right)
$$

is invertible, we infer $\left(\begin{array}{ll}x & y \\ z^{\prime} & t^{\prime}\end{array}\right)$ is invertible.

On the other hand

$$
\left(\begin{array}{cc}
x & y \\
z^{\prime} & t^{\prime}
\end{array}\right)\left(\begin{array}{ll}
a^{\prime} & b^{\prime} \\
c^{\prime} & d^{\prime}
\end{array}\right)=\left(\begin{array}{ll}
w & 0 \\
0 & 1
\end{array}\right)
$$

is invertible, so that $w$ is invertible and we have

$$
\left(\begin{array}{cc}
x & y \\
z^{\prime} & t^{\prime}
\end{array}\right)=\left(\begin{array}{ll}
w & 0 \\
0 & 1
\end{array}\right)\left(\begin{array}{ll}
x^{\prime} & y^{\prime} \\
z^{\prime} & t^{\prime}
\end{array}\right)
$$

which gives $x=w x^{\prime}, y=w y^{\prime}$.

Corollary. The map $\pi \rightarrow \pi^{\perp}$ is well-defined.

Proof. We have two things to check.

First, using the same notation as in the lemma, since

$$
\pi=\left(\begin{array}{ll}
a & b \\
c & d
\end{array}\right) / \widetilde{\lambda n}=\left(\begin{array}{ll}
a^{\prime} & b \\
c^{\prime} & d
\end{array}\right) / \widetilde{\lambda n}
$$

we must show that

$$
\left(\begin{array}{ll}
z^{*} & x^{*} \\
t^{*} & y^{*}
\end{array}\right) / \widetilde{\lambda n}=\left(\begin{array}{ll}
z^{* *} & x^{* *} \\
t^{\prime *} & y^{\prime *}
\end{array}\right) / \widetilde{\lambda n} .
$$

This is indeed so, since $w^{*}$ is invertible and $x^{*} w^{*}=x^{*}, y^{*} w^{*}=y^{\prime *}$.

Secondly if $u$ is invertible and

$$
\left(\begin{array}{ll}
x^{\prime \prime} & y^{\prime \prime} \\
z^{\prime \prime} & t^{\prime \prime}
\end{array}\right)=\left(\begin{array}{ll}
a & b u \\
c & d u
\end{array}\right)^{-1}
$$


then it is easily seen that $x=x^{\prime \prime}, y=y^{\prime \prime}$ and hence clearly

$$
\left(\begin{array}{ll}
z^{\prime \prime *} & x^{\prime \prime *} \\
t^{\prime \prime *} & y^{\prime \prime *}
\end{array}\right) / \widetilde{\lambda n}=\left(\begin{array}{ll}
z^{*} & x^{*} \\
t^{*} & y^{*}
\end{array}\right) / \widetilde{\lambda n}
$$

Remark also that the definition of $\pi^{\perp}$ can also be written

$$
\pi^{\perp}=\left(\left(\begin{array}{ll}
a & b \\
c & d
\end{array}\right)^{*-1}\left(\begin{array}{ll}
0 & 1 \\
1 & 0
\end{array}\right)\right) / \widetilde{\lambda n}=\left(\left(\begin{array}{ll}
a & b \\
c & d
\end{array}\right)\left(\begin{array}{ll}
0 & 1 \\
1 & 0
\end{array}\right)\right)^{*-1} / \widetilde{\lambda n} .
$$

Proposition. We have $\pi^{* *}=\pi$ and $\pi^{\perp \perp}=\pi$. The maps $\pi \rightarrow \pi^{*}$ and $\pi \rightarrow \pi^{\perp}$ are antiholomorphic automorphisms of $G r_{n}(B)$.

Proof. That $\pi^{* *}=\pi$ and $\pi^{\perp \perp}=\pi$ follows immediately using the formula for $\pi^{*}$ and the fact that $\pi^{*}=\left(\begin{array}{cc}0 & 1 \\ -1 & 0\end{array}\right) \pi_{n} \pi^{\perp}$. Because of the definition of $\pi^{*}$ via $\pi^{\perp}$, it suffices to show that $\pi \rightarrow \pi^{\perp}$ is antiholomorphic.

The antiholomorphicity needs only to be checked in charts

$$
\begin{gathered}
\left\{\left(\left(\begin{array}{ll}
a & b \\
c & d
\end{array}\right)\left(\begin{array}{ll}
1 & f \\
0 & 1
\end{array}\right)\right) / \widetilde{\lambda n} \mid f \in \mathfrak{M}_{n}(B)\right\} . \\
\text { If } \pi=\left(\left(\begin{array}{ll}
a & b \\
c & d
\end{array}\right)\left(\begin{array}{ll}
1 & f \\
0 & 1
\end{array}\right)\right) / \widetilde{\lambda n} \text { then } \pi^{\perp}=\left(\left(\begin{array}{ll}
a & b \\
c & d
\end{array}\right)^{*-1}\left(\begin{array}{cc}
1 & 0 \\
-f^{*} & 1
\end{array}\right)\left(\begin{array}{ll}
0 & 1 \\
1 & 0
\end{array}\right)\right) / \widetilde{\lambda n}
\end{gathered}
$$

which clearly is antiholomorphic as a function of $f \in \mathfrak{M}_{n}(B)$.

That the definition of $\pi^{*}$ extends the definition of the involution on the affine space is easily seen. Indeed, then $\pi=\left(\begin{array}{ll}0 & 1 \\ 1 & d\end{array}\right) / \widetilde{\lambda n}$, $\pi^{\perp}=\left(\begin{array}{cc}1 & -d^{*} \\ 0 & 1\end{array}\right) / \widetilde{\lambda n}$ and $\pi^{*}=\left(\begin{array}{cc}0 & 1 \\ -1 & d^{*}\end{array}\right) / \widetilde{\lambda n}$.

We conclude this subsection remarking that in the formula for $\pi^{\perp}$ the matrix $\left(\begin{array}{ll}0 & 1 \\ 1 & 0\end{array}\right)$ can be replaced by $\left(\begin{array}{cc}0 & 1 \\ -1 & 0\end{array}\right)$, since this does not affect the second column in the result. Hence the formula for $\pi^{*}$ can be written also in the form

$$
\pi^{*}=\left(-\left(\begin{array}{cc}
0 & 1 \\
-1 & 0
\end{array}\right)\left(\begin{array}{ll}
a & b \\
c & d
\end{array}\right)^{*-1}\left(\begin{array}{cc}
0 & 1 \\
-1 & 0
\end{array}\right)^{-1}\right) / \widetilde{\lambda n}
$$


Note also that this gives $(C(g) \pi)^{*}=C\left(W g^{*-1} W^{-1}\right) \pi^{*}$ where $W=$ $\left(\begin{array}{cc}0 & 1 \\ -1 & 0\end{array}\right)$ and $g=G L(2, B)$.

9.2. The involution and the bialgebras $\mathcal{A}(\Omega)$. It is easy to see that $(\pi \oplus \sigma)^{*}=\pi^{*} \oplus \sigma^{*}$ and that $(s \cdot \pi)^{*}=s^{*-1} \cdot \pi^{*}$ where $\sigma \in G r_{m}(B)$, $\pi \in G r_{n}(B), s \in G L(n ; \mathbb{C})$.

It follows that if $\Omega=\left(\Omega_{n}\right)_{n \in \mathbb{N}}$ is a fully matricial set of the $B$ Grassmannian then the same holds for $\Omega^{*}=\left(\Omega_{n}^{*}\right)_{n \in \mathbb{N}}$, where $\Omega_{n}^{*}=$ $\left\{\pi^{*} \mid \pi \in \Omega_{n}\right\}$. Clearly $\Omega$ is open iff $\Omega^{*}$ is open.

If $f_{n}: \Omega_{n} \rightarrow \mathfrak{M}_{n}$, we define $f_{n}^{*}: \Omega_{n}^{*} \rightarrow \mathfrak{M}_{n}$ by $\left(f_{n}(\pi)\right)^{*}=f_{n}^{*}\left(\pi^{*}\right)$, where $\pi \in \Omega_{n}$. If $f_{n}$ is analytic then so is $f_{n}^{*}$ and if $f=\left(f_{n}\right)_{n \in \mathbb{N}} \in \mathcal{A}(\Omega)$ then $f^{*}=\left(f_{n}^{*}\right)_{n \in \mathbb{N}} \in \mathcal{A}(\Omega)$ and the map $f \rightarrow f^{*}$ is a conjugate-linear antiisomorphism. More generally there is a conjugate-linear antiisomorphism $f \rightarrow f^{*}$ of $\mathcal{A}\left(\Omega_{1} ; \Omega_{2} ; \ldots ; \Omega_{p}\right)$ and $\mathcal{A}\left(\Omega_{1}^{*}, \Omega_{2}^{*} ; \ldots ; \Omega_{p}^{*}\right)$ where

$$
f_{n_{1}, \ldots, n_{p}}^{*}\left(\omega_{1}^{*} ; \ldots ; \omega_{p}^{*}\right)=\left(f_{n_{1}, \ldots, n_{p}}\left(\omega_{1} ; \ldots ; \omega_{p}\right)\right)^{*} .
$$

If $\Omega=\Omega^{*}$ then $\mathcal{A}(\Omega)$ is an algebra with involution. More generally $\mathcal{A}\left(\Omega_{1} ; \ldots ; \Omega_{p}\right)$ is an algebra with involution when $\Omega_{j}=\Omega_{j}^{*}, 1 \leq j \leq p$.

To state the property of $\widetilde{\partial}$ with respect to the involution we will denote by

$$
\sigma_{1,2}: \mathcal{A}(\Omega ; \Omega) \rightarrow \mathcal{A}(\Omega ; \Omega)
$$

the automorphism permuting the variables, that is

$$
\left(\sigma_{1,2} f\right)_{m, n}(\sigma, \pi)=\varepsilon_{m, n} \circ f_{n, m}(\pi, \sigma)
$$

with $\varepsilon_{m, n}: \mathfrak{M}_{m} \otimes \mathfrak{M}_{n} \rightarrow \mathfrak{M}_{n} \otimes \mathfrak{M}_{m}$ the tensorial permutation isomorphism.

Proposition. If $f \in \mathcal{A}(\Omega)$, then

$$
\widetilde{\partial} f^{*}=\sigma_{1,2}(\widetilde{\partial} f)^{*} .
$$

In particular if $\Omega=\Omega^{*}$, this is the compatibility of the involution and comultiplication of $\mathcal{A}(\Omega)$.

Proof. If $L \in \mathcal{L}\left(\mathfrak{M}_{m, n}\right)$ and $L^{*} \in \mathcal{L}\left(\mathfrak{M}_{n, m}\right)$ is defined by $L^{*}(y)=$ $\left(L\left(y^{*}\right)\right)^{*}$ then

$$
\varepsilon_{m, n}\left(\left(\alpha_{m, n}(L)\right)^{*}\right)=\alpha_{n, m}\left(L^{*}\right) .
$$


In view of this it is easily seen that the proposition will follow if we prove that:

$$
\begin{aligned}
& f_{m, n}^{*}\left(\left(\Gamma\left(\begin{array}{cccc}
a & b & 0 & 0 \\
c & d & 0 & t b^{\prime} \\
0 & 0 & a^{\prime} & b^{\prime} \\
0 & 0 & c^{\prime} & d^{\prime}
\end{array}\right) \Gamma^{-1}\right) / \widetilde{\lambda m+n}\right) \\
& =S\left(f_{n+m}\left(\left(\Gamma^{\prime}\left(\begin{array}{cccc}
\alpha^{\prime} & \beta^{\prime} & 0 & 0 \\
\gamma^{\prime} & \delta^{\prime} & 0 & t^{*} \beta \\
0 & 0 & \alpha & \beta \\
0 & 0 & \gamma & \delta
\end{array}\right) \Gamma^{\prime-1}\right) / \widetilde{\lambda n+m}\right) S^{-1} S^{*}\right.
\end{aligned}
$$

where $\Gamma, \Gamma^{\prime}, S$ are permutation matrices, the first two having the effect by conjugation of permuting second and third rows and columns in $4 \times 4$ block matrices and $S$ permuting first and second rows and columns in a $2 \times 2$ block matrix (the sizes of blocks corresponding to $m+m+n+n$, $n+n+m+m$ and $n+m$ respectively). The other notations used are $t \in \mathfrak{M}_{m, n}(\mathbb{C})$ and

$$
\begin{aligned}
& \left(\left(\begin{array}{ll}
a & b \\
c & d
\end{array}\right) / \widetilde{\lambda m}\right)^{*}=\left(\begin{array}{ll}
\alpha & \beta \\
\gamma & \delta
\end{array}\right) / \widetilde{\lambda m} \\
& \left(\left(\begin{array}{ll}
a^{\prime} & b^{\prime} \\
c^{\prime} & d^{\prime}
\end{array}\right) / \widetilde{\lambda n}\right)^{*}=\left(\begin{array}{ll}
\alpha^{\prime} & \beta^{\prime} \\
\gamma^{\prime} & \delta^{\prime}
\end{array}\right) / \widetilde{\lambda n} .
\end{aligned}
$$

Remark that the right-hand side of the equality to be proved is equal to

$$
\left(f_{n+m}\left(S \cdot\left(\Gamma^{\prime}\left(\begin{array}{cccc}
\alpha^{\prime} & \beta^{\prime} & 0 & 0 \\
\gamma^{\prime} & \delta^{\prime} & 0 & t^{*} \beta \\
0 & 0 & \alpha & \beta \\
0 & 0 & \gamma & \delta
\end{array}\right) \Gamma^{\prime-1}\right) / \widehat{\lambda n+m}\right)\right)^{*}
$$

Hence by the definition of $f_{m+n}^{*}$ it will suffice to show that

$$
\begin{aligned}
& \left(\left(\Gamma\left(\begin{array}{cccc}
a & b & 0 & 0 \\
c & d & 0 & t b^{\prime} \\
0 & 0 & a^{\prime} & b^{\prime} \\
0 & 0 & c^{\prime} & d^{\prime}
\end{array}\right) \Gamma^{-1}\right) / \widetilde{\lambda m+n}\right)^{*} \\
& =S \cdot\left(\Gamma^{\prime}\left(\begin{array}{cccc}
\alpha^{\prime} & \beta^{\prime} & 0 & 0 \\
\gamma^{\prime} & \delta^{\prime} & 0 & t^{*} \beta \\
0 & 0 & \alpha & \beta \\
0 & 0 & \gamma & \delta
\end{array}\right) \Gamma^{\prime-1}\right) / \widehat{\lambda m+n} .
\end{aligned}
$$

Writing the equality in the form $A / \widehat{\lambda m+n}=B / \widehat{\lambda m+n}$ the problem amounts to showing that $A^{-1} B$ is a lower triangular $2 \times 2$ block matrix 
with invertible diagonal blocks. Denoting by $\Theta$ and $\Xi$ the $4 \times 4$ explicitly written matrices in $A$ and $B$ and by $\Sigma$ and $W$ the matrices $\left(\begin{array}{ll}S & 0 \\ 0 & S\end{array}\right)$ and $\left(\begin{array}{cc}0 & I \\ -I & 0\end{array}\right)$, we have:

$$
\begin{aligned}
& A=-W \Gamma \Theta^{*-1} \Gamma^{-1} W^{-1} \\
& B=\Sigma \Gamma^{\prime} \Xi \Gamma^{-1} \Sigma^{-1} .
\end{aligned}
$$

Hence

$$
\begin{aligned}
A^{-1} B & =-W \Gamma \Theta^{*} \Gamma^{-1} W^{-1} \Sigma \Gamma^{\prime} \Xi \Gamma^{-1} \Sigma^{-1} \\
& =-W \Gamma \Theta^{*} U \Xi U^{-1} \Gamma^{-1} W^{-1}
\end{aligned}
$$

where $U=\Gamma^{-1} W^{-1} \Sigma \Gamma^{\prime}$. It is easily seen that

$$
U=\left(\begin{array}{cccc}
0 & 0 & 0 & -I_{m} \\
0 & 0 & I_{m} & 0 \\
0 & -I_{m} & 0 & 0 \\
I_{n} & 0 & 0 & 0
\end{array}\right)
$$

and hence that

$$
U \Xi U^{-1}=\left(\begin{array}{cccc}
\delta & -\gamma & 0 & 0 \\
-\beta & \alpha & 0 & 0 \\
t^{*} \beta & 0 & \delta^{\prime} & -\gamma^{\prime} \\
0 & 0 & -\beta^{\prime} & \alpha^{\prime}
\end{array}\right)
$$

To compute $\Xi^{*} U \Xi U^{-1}$ remark first that in view of the formula for $\pi^{*}$, we may assume

$$
\left(\begin{array}{ll}
a & b \\
c & d
\end{array}\right)^{-1}=\left(\begin{array}{cc}
-\delta^{*} & \beta^{*} \\
\gamma^{*} & -\alpha^{*}
\end{array}\right)
$$

as well as the primed analogue of this. Equivalently we have

$$
\begin{aligned}
\left(\begin{array}{cc}
\delta & -\gamma \\
-\beta & \alpha
\end{array}\right)^{-1} & =-\left(\begin{array}{ll}
a^{*} & c^{*} \\
b^{*} & d^{*}
\end{array}\right) \\
& =-\left(\begin{array}{ll}
a & b \\
c & d
\end{array}\right)^{*}
\end{aligned}
$$

and its primed analogue. We get

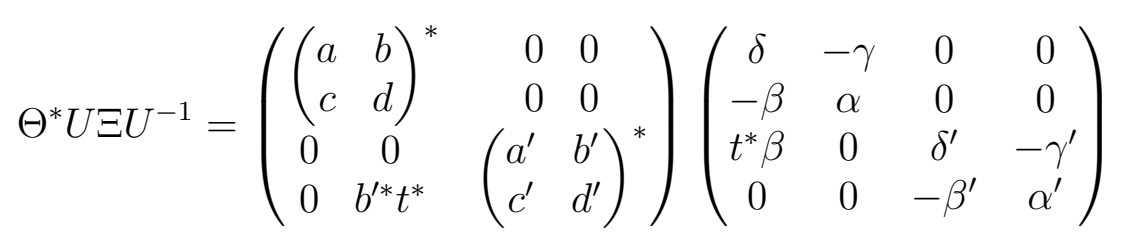




$$
=\left(\begin{array}{cccc}
I & 0 & 0 & 0 \\
0 & I & 0 & 0 \\
a^{\prime *} t^{*} \beta & 0 & I & 0 \\
0 & b^{* *} t^{*} \alpha & 0 & I
\end{array}\right) .
$$

This in turn gives

$$
\begin{aligned}
-W \Gamma\left(\Theta^{*} U \Xi U^{-1}\right) \Gamma^{-1} W^{-1} & =-W\left(\begin{array}{cccc}
I & 0 & 0 & 0 \\
a^{\prime *} t^{*} \beta & I & 0 & 0 \\
0 & 0 & I & 0 \\
0 & 0 & b^{\prime *} t^{*} \alpha & I
\end{array}\right) W^{-1} \\
& =-\left(\begin{array}{cccc}
I & 0 & 0 & 0 \\
b^{\prime *} t^{*} \alpha & I & 0 & 0 \\
0 & 0 & I & 0 \\
0 & 0 & a^{\prime *} t^{*} \beta & I
\end{array}\right)
\end{aligned}
$$

which is a matrix of the desired kind.

9.3. The involution and the coderivation $\Lambda$. In this subsection we check the compatibility of $\Lambda$ with the involution.

Proposition. If $f \in \mathcal{A}(\Omega)$ then we have

$$
\Lambda f^{*}=(\Lambda f)^{*}
$$

(the same $\Lambda$ denotes the coderivations in $\mathcal{A}(\Omega)$ and in $\mathcal{A}\left(\Omega^{*}\right)$ ).

Proof. If $\pi=\left(\begin{array}{ll}a & b \\ c & d\end{array}\right) / \widetilde{\lambda n} \in \Omega_{n}$, then

$$
\begin{aligned}
\left(\left(\begin{array}{cc}
1 & 0 \\
0 & e^{t}
\end{array}\right)_{n} \pi\right)^{*} & =\left(\begin{array}{cc}
0 & 1 \\
-1 & 0
\end{array}\right)_{n}\left(\begin{array}{cc}
1 & 0 \\
0 & e^{t}
\end{array}\right)_{n}^{*-1}\left(\begin{array}{cc}
0 & 1 \\
-1 & 0
\end{array}\right)^{-1} \pi^{*} \\
& =\left(\begin{array}{cc}
e^{-t} & 0 \\
0 & 1
\end{array}\right)_{n} \pi^{*}=\left(\begin{array}{ll}
1 & 0 \\
0 & e^{t}
\end{array}\right)_{n} \pi^{*}
\end{aligned}
$$

from which the proposition follows immediately using the formula for $\Lambda$.

9.4. The involution and Grassmannian resolvents. In this subsection we check the behavior of resolvents with respect to the involution.

We will need an algebraic lemma which provides explicit formulae for resolvents.

Lemma. Assume that, with entries in some unital ring, we have $\left(\begin{array}{ll}a & b \\ c & d\end{array}\right)^{-1}=\left(\begin{array}{ll}x & y \\ z & t\end{array}\right)$ and $\left(\begin{array}{ll}\alpha & \beta \\ \gamma & \delta\end{array}\right)^{-1}=\left(\begin{array}{ll}r & s \\ u & v\end{array}\right)$. Then the matrix 
$\left(\begin{array}{ll}b & \beta \\ d & \delta\end{array}\right)$ is invertible iff $x \beta+y \delta$ is invertible, which is also iff $r b+$ sd is invertible. Moreover we then have:

$$
\left(\begin{array}{ll}
b & \beta \\
d & \delta
\end{array}\right)^{-1}=\left(\begin{array}{cc}
(r b+s d)^{-1} r & (r b+s d)^{-1} s \\
(x \beta+y \delta)^{-1} x & (x \beta+y \delta)^{-1} y
\end{array}\right) .
$$

Proof. Since

$$
\left(\begin{array}{ll}
x & y \\
z & t
\end{array}\right)\left(\begin{array}{ll}
b & \beta \\
d & \delta
\end{array}\right)=\left(\begin{array}{cc}
0 & x \beta+y \delta \\
1 & z \beta+t \delta
\end{array}\right)
$$

we get the "iff $x \beta+y \delta$ is invertible" part of the statement and

$$
\begin{aligned}
\left(\begin{array}{ll}
b & \beta \\
d & \delta
\end{array}\right)^{-1} & =\left(\begin{array}{cc}
0 & x \beta+y \delta \\
1 & z \beta+t \delta
\end{array}\right)^{-1}\left(\begin{array}{cc}
x & y \\
z & t
\end{array}\right) \\
& =\left(\begin{array}{cc}
* & * \\
(x \beta+y \delta)^{-1} & 0
\end{array}\right)\left(\begin{array}{cc}
x & y \\
z & t
\end{array}\right) \\
& =\left(\begin{array}{cc}
* & * \\
(x \beta+y \delta)^{-1} x & (x \beta+y \delta)^{-1} y
\end{array}\right) .
\end{aligned}
$$

Similarly, since

$$
\left(\begin{array}{ll}
r & s \\
u & v
\end{array}\right)\left(\begin{array}{ll}
b & \beta \\
d & \delta
\end{array}\right)=\left(\begin{array}{ll}
r b+s d & 0 \\
u b+v d & 1
\end{array}\right)
$$

we get the "iff $r b+s d$ is invertible" part of the statement and

$$
\begin{aligned}
\left(\begin{array}{ll}
b & \beta \\
d & \delta
\end{array}\right)^{-1} & =\left(\begin{array}{cc}
r b+s d & 0 \\
u b+v d & 1
\end{array}\right)^{-1}\left(\begin{array}{ll}
r & s \\
u & v
\end{array}\right) \\
& =\left(\begin{array}{cc}
(r b+s d)^{-1} & 0 \\
* & *
\end{array}\right)\left(\begin{array}{ll}
r & s \\
u & v
\end{array}\right) \\
& =\left(\begin{array}{cc}
(r b+s d)^{-1} r & (r b+s d)^{-1} s \\
* & *
\end{array}\right) .
\end{aligned}
$$

The framework for resolvents will be a unital Banach algebra with involution $E$ and a Banach subalgebra with the same involution $1 \in$ $B \subset E$.

Proposition. Let $\pi \in G r_{1}(E)$ and $\sigma \in G r_{n}(B)$ be such that $\sigma \in$ $\widetilde{\rho}_{n}(\pi ; B)$. Then $\sigma^{*} \in \widetilde{\rho}_{n}\left(\pi^{*} ; B\right)$ and $\left(\widetilde{\mathcal{R}}_{n}(\pi ; B)(\sigma)\right)^{*}=\widetilde{\mathcal{R}}_{n}\left(\pi^{*} ; B\right)\left(\sigma^{*}\right)$.

Proof. Remark that it suffices to prove the proposition when $B=E$ and $n=1$. Indeed, replacing $E$ by $\mathfrak{M}_{n}(E)$ we get the reduction to the case $n=1$. 
Let $\pi=\left(\begin{array}{ll}a & b \\ c & d\end{array}\right) / \widetilde{\lambda 1}, \sigma=\left(\begin{array}{ll}\alpha & \beta \\ \gamma & \delta\end{array}\right) / \widetilde{\lambda 1}$ and use the notation for the inverses of the two matrices which we used in the lemma. Then we have $\widetilde{\mathcal{R}}_{1}(\pi ; E)(\sigma)=\beta(x \beta+y \delta)^{-1} y$ and $\sigma \in \widetilde{\rho}_{1}(\pi ; E)$ is equivalent to the invertibility of $x \beta+y \delta$ by the lemma.

On the other hand we have

$$
\begin{aligned}
\pi^{*} & =\left(\begin{array}{cc}
-t^{*} & y^{*} \\
z^{*} & -x^{*}
\end{array}\right) / \widetilde{\lambda 1} \\
\sigma^{*} & =\left(\begin{array}{cc}
-v^{*} & s^{*} \\
u^{*} & -r^{*}
\end{array}\right) / \widetilde{\lambda 1}
\end{aligned}
$$

and

$$
\left(\begin{array}{cc}
-t^{*} & y^{*} \\
z^{*} & -x^{*}
\end{array}\right)^{-1}=\left(\begin{array}{cc}
-d^{*} & b^{*} \\
c^{*} & -a^{*}
\end{array}\right)
$$

Applying again the lemma, to these new matrices, we get that $\sigma^{*} \in$ $\widetilde{\rho}_{1}\left(\pi^{*} ; E\right)$ is equivalent to the invertibility of $-d^{*} s^{*}-b^{*} r^{*}$ and $\widetilde{\mathcal{R}}_{1}\left(\pi^{*} ; E\right)\left(\sigma^{*}\right)=$ $-s^{*}\left(d^{*} s^{*}+b^{*} r^{*}\right)^{-1} b^{*}$. Since $-d^{*} s^{*}-b^{*} r^{*}$ is invertible iff $r b+s d$ is invertible, the equivalence of $\sigma^{*} \in \widetilde{\rho}_{1}\left(\pi^{*} ; E\right)$ with $\sigma \in \widetilde{\rho}_{1}(\pi ; E)$ is precisely the equivalence of the invertibility of $r b+s d$ and of $x \beta+y d$.

To conclude the proof of the proposition we must show that

$$
\left(\beta(x \beta+y \delta)^{-1} y\right)^{*}+s^{*}\left(d^{*} s^{*}+b^{*} r^{*}\right)^{-1} b^{*}=0,
$$

or equivalently, that

$$
\beta(x \beta+y \delta)^{-1} y+b(r b+s d)^{-1} s=0 .
$$

This is a consequence of the last assertion of the lemma, which gives that

$$
\left(\begin{array}{ll}
b & \beta \\
d & \delta
\end{array}\right)\left(\begin{array}{cc}
(r b+s d)^{-1} r & (r b+s d)^{-1} s \\
(x \beta+y \delta)^{-1} x & (x \beta+y \delta)^{-1} y
\end{array}\right)=\left(\begin{array}{cc}
* & 0 \\
* & *
\end{array}\right) .
$$

9.5. The involution and the duality transform. Like in the previous section $1 \in B \subset E$ will be Banach algebras with involution. Since we will consider the duality transforms with respect to $\pi, B$ and with respect to $\pi^{*}, B$ we will use the notations $\mathcal{U}_{\pi}(\cdot)$ and respectively $\mathcal{U}_{\pi^{*}}(\cdot)$ to distinguish the two.

Proposition. We have $(\mathcal{L} \mathcal{R}(\pi ; B))^{*}=\mathcal{L} \mathcal{R}\left(\pi^{*} ; B\right)$ and $\left(\mathcal{U}_{\pi}(\varphi)\right)^{*}=$ $\mathcal{U}_{\pi^{*}}\left(\varphi^{*}\right)$.

The proof is a straightforward consequence of Proposition 9.4 and of the definitions of $\mathcal{L} \mathcal{R}(\pi ; B)$ and of the duality transform and will therefore be omitted. 


\section{Dual Positivity}

10.1. The Definition. The Grassmannian extension of the notion of dual positivity is quite straightforward. Here $B$ will be a unital Banach algebra with involution.

Definition. If $\Omega=\Omega^{*}$ an element $f \in \mathcal{A}(\Omega)$ is dual-positive if $f=f^{*}$ and $\nabla_{n, n} f\left(\sigma, \sigma^{*}\right)$ is a positive map of $\mathfrak{M}_{n}$ into $\mathfrak{M}_{n}$ for all $\sigma \in \Omega_{n}$ and $n \in \mathbb{N}\left(\nabla_{m, n} f\left(\sigma^{\prime}, \sigma^{\prime \prime}\right)\right.$ denotes the map $\left.\alpha_{m, n} \widetilde{\partial}_{m, n} f\left(\sigma^{\prime}, \sigma^{\prime \prime}\right)\right)$.

Like in the affine case we have a few equivalent conditions.

Proposition. If $\Omega=\Omega^{*}$ and $f \in \mathcal{A}(\Omega)$, the following are equivalent:

(i) $f$ is dual positive

(ii) $f=f^{*}$ and for any $\sigma^{(j)} \in \Omega_{n(j)}, 1 \leq j \leq p, \bigoplus_{1 \leq i, j \leq p}\left(\nabla_{n(i), n(j)} f\right)\left(\sigma^{(i)}, \sigma^{(j) *}\right)$ is a positive linear map of $\bigoplus_{i, j} \mathfrak{M}_{n(i), n(j)}$, identiied with $\mathfrak{M}_{n(1)+\cdots+n(p)}$ into itself.

(iii) $f=f^{*}$ and for any $\sigma \in \Omega_{n}$, the $\operatorname{map}\left(\nabla_{n, n} f\right)\left(\sigma, \sigma^{*}\right): \mathfrak{M}_{n} \rightarrow \mathfrak{M}_{n}$ is completely positive.

The proof from the affine case [16, Prop. 8.2] immediately carries over to this more general case and will not be repeated.

10.2. The duality transforms of the positive functionals. Here $1 \in B \subset E$ will be an inclusion of unital Banach algebras with involution. By $E_{1}$ we shall denote the closure of $\mathcal{L} \mathcal{R}(\pi ; B)$ where $\pi=\pi^{*} \in$ $G r_{1}(E)$. A functional $\varphi \in E_{1}^{d}$ is positive, denoted $\varphi \geq 0$, if $\varphi=\varphi^{*}$ and $\varphi\left(y^{*} y\right) \geq 0$ for all $y \in E_{1}$ (the hermiticity follows actually from the second requirement).

Proposition. If $\varphi \in E_{1}^{d}$, then $\varphi \geq 0$ iff $-\mathcal{U}(\varphi)$ is dual positive in $\mathcal{A}(\widetilde{\rho}(\pi ; B))$.

Proof. a) $\varphi \geq 0 \Rightarrow-\mathcal{U}(\varphi)$ dual positive. We shall use Proposition 6.3, which implies that

$$
\left(\operatorname{id}_{\mathfrak{M}_{n}} \otimes \operatorname{id}_{\mathfrak{M}_{n}} \otimes \varphi\right)\left(\widetilde{\mathcal{R}}(\pi ; B)(\sigma) \otimes_{E} \widetilde{\mathcal{R}}(\pi ; B)\left(\sigma^{*}\right)\right)=-\widetilde{\partial}_{n, n} \mathcal{U}(\varphi)\left(\sigma, \sigma^{*}\right) .
$$

Since $\pi=\pi^{*}$, we have

$$
\widetilde{\mathcal{R}}(\pi ; B)(\sigma)=\left(\widetilde{\mathcal{R}}\left(\pi^{*} ; B\right)\left(\sigma^{*}\right)\right)^{*} .
$$

Hence, if $x_{i j} \in E_{1}$ are such that $\widetilde{\mathcal{R}}(\pi ; B)(\sigma)=\sum_{i, j} e_{i j} \otimes x_{i j}$ then

$$
-\widetilde{\partial}_{n, n} \mathcal{U}(\varphi)\left(\sigma ; \sigma^{*}\right)=\sum_{1 \leq i, j, k, l \leq n} \varphi\left(x_{i j} x_{l k}^{*}\right) e_{i j} \otimes e_{k l} .
$$


We must check that

$$
\alpha_{n, n}\left(-\widetilde{\partial}_{n, n} \mathcal{U}(\varphi)\left(\sigma ; \sigma^{*}\right)\right)\left(\sum_{p, q} c_{p} \bar{c}_{q} e_{p q}\right) \geq 0 .
$$

In view of the definition of $\alpha$, this is equivalent to

$$
\sum_{1 \leq i, j, k, l \leq n} c_{j} \bar{c}_{k} \varphi\left(x_{i j} x_{l k}^{*}\right) e_{i l} \geq 0 .
$$

Let $a_{i}=\sum_{1 \leq j \leq n} c_{j} x_{i j} \in E_{1}$. Then the inequality we must prove, becomes

$$
\sum_{1 \leq i, l \leq n} \varphi\left(a_{i} a_{l}^{*}\right) e_{i l} \geq 0
$$

or equivalently, for all $\lambda_{1}, \ldots, \lambda_{n} \in \mathbb{C}$

$$
\sum_{1 \leq i, l \leq n} \varphi\left(a_{i} a_{l}^{*}\right) \lambda_{i} \bar{\lambda}_{l} \geq 0
$$

Putting $y=\sum_{1 \leq i \leq n} \lambda_{i} a_{i}$, we get $\varphi\left(y y^{*}\right) \geq 0$, which is indeed so.

b) $-\mathcal{U}(\varphi)$ dual positive $\Rightarrow \varphi \geq 0$. We have

$$
\left(\left(-\nabla_{n, n} \mathcal{U}(\varphi)\left(\sigma, \sigma^{*}\right)\right) e_{j k}\right)_{i l}=\varphi\left(\left(\widetilde{\mathcal{R}}_{n}(\pi ; B)(\sigma)\right)_{i j}\left(\widetilde{\mathcal{R}}_{n}(\pi ; B)(\sigma)\right)_{l k}^{*}\right) .
$$

We must show that $\varphi\left(\xi \xi^{*}\right) \geq 0$ if $\xi \in \mathcal{L} \mathcal{R}(\pi ; B)$. Using instead of $\sigma^{\prime}, \sigma^{\prime \prime}, \sigma^{\prime \prime \prime}, \ldots$ the direct sum $\sigma=\sigma^{\prime} \oplus \sigma^{\prime \prime} \oplus \sigma^{\prime \prime \prime} \oplus$. . we can assume $\xi$ is a linear combination of matrix entries of $\widetilde{\mathcal{R}}_{n}(\pi ; B)(\sigma)$. So, we must show that

$$
\sum_{1 \leq i, j, k, l \leq n} c_{i j} \bar{l}_{l k} \varphi\left(\left(\widetilde{\mathcal{R}}_{n}(\pi ; B)(\sigma)\right)_{i j}\left(\widetilde{\mathcal{R}}_{n}(\pi ; B)(\sigma)\right)_{l k}^{*}\right) \geq 0
$$

which is equivalent to

$$
\left.\sum_{1 \leq i, j, k, l \leq n}\left(-\nabla_{n, n} \mathcal{U}(\varphi)\left(\sigma ; \sigma^{*}\right)\right)\left(e_{j k}\right)\right)_{i l} c_{i j} \bar{c}_{l k} \geq 0
$$

Let $\Phi=-\nabla_{n, n} \mathcal{U}(\varphi)\left(\sigma ; \sigma^{*}\right)$. The complete positivity of $\Phi$ gives that

$$
0 \leq \Theta=\sum_{1 \leq j, k \leq n} \Phi\left(e_{j k}\right) \otimes e_{j k}=\sum_{1 \leq i, j, k, l}\left(\Phi\left(e_{j k}\right)\right)_{i l} e_{i l} \otimes e_{j k} .
$$

If $e_{l}$ is the canonical basis in $\mathbb{C}^{n}$ and $\eta=\sum_{1 \leq l, k \leq n} \bar{c}_{l k} e_{l} \otimes e_{k} \in \mathbb{C}^{n} \otimes \mathbb{C}^{n}$, then

$$
0 \leq\langle\Theta \eta, \eta\rangle=\sum_{1 \leq i, j, k, l \leq n}\left(\Phi\left(e_{j k}\right)\right)_{i l} c_{i j} \bar{c}_{l k}
$$

which concludes the proof. 


\section{Stably Matricial Sets, Matricial Half-Planes and UNIT BALLS}

11.1. Stably matricial sets. Matricial half-planes, unit balls, which are fundamental in operator theory, don't satisfy the similarity requirement of fully matricial sets. These matricial sets have somewhat weaker properties, and will be called stably matricial. Stably matricial sets can easily be turned into fully matricial sets. Since affine space is part of the Grassmannian we will only give the Grassmannian definition, though, the easy adaptation to the affine case, will often be easier to work with.

Definition. a) If $\Xi=\left(\Xi_{n}\right)_{n \in \mathbb{N}}$, where $\Xi_{n} \subset G r_{n}(B)$ we will call $\Xi$ a stably matricial B-set of the Grassmannian if the following two conditions are satisfied.

$1^{\circ}$ if $\pi \in G r_{m}(B), \sigma \in G r_{n}(B)$ then $\pi \oplus \sigma \in \Xi_{m+n} \Leftrightarrow \pi \in \Xi_{m}$, $\sigma \in \Xi_{n}$.

$2^{\circ}$ if $\pi \in G r_{m}(B), \sigma \in G r_{n}(B)$ and $s \in G L(m+n ; \mathbb{C})$ is such that $s \cdot(\pi \oplus \sigma) \in \Xi_{m+n}$, then there are $s^{\prime} \in G L(m ; \mathbb{C}), s^{\prime \prime} \in G L(n ; \mathbb{C})$, so that $s^{\prime} \cdot \pi \in \Xi_{m}, s^{\prime \prime} \cdot \sigma \in \Xi_{n}$.

b) If $\Xi=\left(\Xi_{n}\right)_{n \in \mathbb{N}}$ is stably matricial and $f=\left(f_{n}\right)_{n \in \mathbb{N}}$, where $f_{n}: \Xi_{n} \rightarrow \mathfrak{M}_{n}$, then we will say $f$ is a stably matricial function on $\Xi$ if

$1^{\circ} f_{m+n}(\pi \oplus \sigma)=f_{m}(\pi) \oplus f_{n}(\sigma)$ when $\pi \in \Xi_{m}, \sigma \in \Xi_{n}$.

$2^{\circ}$ if $\pi, \pi^{\prime} \in \Xi_{n}$ and $s \in G L_{n}(\mathbb{C})$ is such that $s \cdot \pi=\pi^{\prime}$, then Ad $s\left(f_{n}(\pi)\right)=f_{n}\left(\pi^{\prime}\right)$.

Remark. The reader can easily adapt part b) of the definition to functions with $f_{n}: \Xi_{n} \rightarrow \mathfrak{M}_{n}(\mathcal{X})$, where $\mathcal{X}$ is some Banach space or even more generally $f_{n}: \Xi_{n} \rightarrow \Theta_{n}$, where $\Theta=\left(\Theta_{n}\right)_{n \in \mathbb{N}}$ is a stably matricial $A$-set, where $A$ is some Banach algebra. In another direction, there are several variables stably matricial functions. For instance for two variables we consider $f=\left(f_{m, n}\right)_{(m, n) \in \mathbb{N}^{2}}, f_{m, n}: \Xi_{m} \times \Xi_{n} \rightarrow$ $\mathfrak{M}_{m} \otimes \mathfrak{M}_{n}$ satisfying: $f_{m^{\prime}+m^{\prime \prime}, n^{\prime}+n^{\prime \prime}}\left(\pi^{\prime} \oplus \pi^{\prime \prime}, \sigma^{\prime} \oplus \sigma^{\prime \prime}\right)=f_{m^{\prime}, n^{\prime}}\left(\pi^{\prime}, \sigma^{\prime}\right) \oplus$ $f_{m^{\prime \prime}, n^{\prime}}\left(\pi^{\prime \prime}, \sigma^{\prime}\right) \oplus f_{m^{\prime}, n^{\prime \prime}}\left(\pi^{\prime}, \sigma^{\prime \prime}\right) \oplus f_{m^{\prime \prime}, n^{\prime \prime}}\left(\pi^{\prime \prime}, \sigma^{\prime \prime}\right)$ and the similarity condition $\left(\operatorname{Ad} s_{1} \otimes \operatorname{Ad} s_{2}\right) f_{m, n}(\pi, \sigma)=f_{m, n}\left(\pi^{\prime}, \sigma^{\prime}\right)$ when $\pi, \pi^{\prime} \in \Xi_{m}, \sigma, \sigma^{\prime} \in \Xi_{n}$ and $s_{1} \in G L(m, \mathbb{C}), s_{2} \in G L(n ; \mathbb{C})$ are such that $s_{1} \cdot \pi=\pi^{\prime}, s_{2} \cdot \sigma=\sigma^{\prime}$. The corresponding extensions of part b) of the next proposition is also an easy exercise left to the reader.

Proposition. a)If $\Xi=\left(\Xi_{n}\right)_{n \in \mathbb{N}}$ is a stably matricial $B$-set of the Grassmannian, then $\widetilde{\Xi}=\left(\widetilde{\Xi}_{n}\right)_{n \in \mathbb{N}}$, where $\widetilde{\Xi}_{n}=G L(n ; \mathbb{C}) \cdot \Xi_{n}$, is a fully 
matricial B-set of the Grassmannian. Moreover, if $\Xi$ is open, then $\widetilde{\Xi}$ is also open.

b)If $f=\left(f_{n}\right)_{n \in \mathbb{N}}$ is a stably matricial function ( $f_{n}$ here is $\mathfrak{M}_{n^{-}}$ valued), then there is a unique extension $\widetilde{f}=\left(\widetilde{f}_{n}\right)_{n \in \mathbb{N}}$ to $\widetilde{\Xi}$ so that $\tilde{f}_{n}(s \cdot \pi)=\operatorname{Ad} s\left(\tilde{f}_{n}(\pi)\right)$. The extension $\tilde{f}$ is fully matricial. Moreover if $\Xi$ is open and $f$ is analytic, then $\tilde{f}$ is fully matricial analytic. Similar statements hold for the more general $f$ considered in the remark preceding the proposition.

c) If $\Xi$ is a stably matricial B-set of the Grassmannian and $g \in$ $G L(2 ; B)$, then $C(g) \Xi$ is also stably matricial and the map $\pi \rightarrow C(g) \pi$ defines a stably matricial isomorphism of $\Xi$ and $C(g) \Xi$, which is analytic when $\Xi$ is open.

The proof is quite straightforward checking and will be left to the reader.

Corollary. If $\Xi, \widetilde{\Xi}$ are like in the proposition then the restriction maps $\mathcal{A}(\widetilde{\Xi}) \rightarrow \mathcal{A}(\Xi), \mathcal{A}(\widetilde{\Xi}, \widetilde{\Xi}) \rightarrow \mathcal{A}(\Xi, \Xi)$ (the $\mathcal{A}(\Xi), \mathcal{A}(\Xi, \Xi)$ denoting the analytic stably matricial functions in the case of stably matricial sets) are bijective. In particular $\mathcal{A}(\Xi)$ becomes via the isomorphism a bialgebra with coderivation $\Lambda$, isomorphic to $\mathcal{A}(\widetilde{\Xi})$.

11.2. The stably matricial unit disk $\mathcal{D}_{0}(B)$. Starting with this subsection and throughout the rest of section 11 we shall assume $B$ is a unital $C^{*}$-algebra.

Let $\mathcal{D}_{0}(B)_{n}=\left\{T \in \mathfrak{M}_{n}(B) \mid\|T\|<1\right\}$ be the open unit ball of $\mathfrak{M}_{n}(B)$ with respect to the $C^{*}$-norm and let $\mathcal{D}_{0}^{c l}(B)_{n}$ be the closed unit ball. We shall also denote by $\mathcal{U}(n ; B)$ the unitary group of $\mathfrak{M}_{n}(B)$. Of course being subsets of $\mathfrak{M}_{n}(B)$ these sets are also subsets of $G r_{n}(B)$.

Proposition. The matricial sets $\mathcal{D}_{0}(B)=\left(\mathcal{D}_{0}(B)_{n}\right)_{n \in \mathbb{N}}, \mathcal{D}_{0}^{c l}(B)=$ $\left(\mathcal{D}_{0}(B)_{n}^{c l}\right)_{n \in \mathbb{N}}$ and $\mathcal{U}(B)=(U(n ; B))_{n \in \mathbb{N}}$ are stably matricial B-sets. Also, if $g_{1}, \ldots, g_{k} \in G L(2 ; B)$, then more generally

$C\left(g_{1}\right) \mathcal{D}_{0}(B) \cap \cdots \cap C\left(g_{k}\right) \mathcal{D}_{0}(B)=\left(C\left(g_{1}\right) \mathcal{D}_{0}(B)_{n} \cap \cdots \cap C\left(g_{k}\right) \mathcal{D}_{0}(B)_{n}\right)_{n \in \mathbb{N}}$

is an open stably matricial set.

Proof. The only part of the statement which is perhaps not immediately clear is property $2^{\circ}$ of part a) of Definition 11.1. In view of Proposition 11.1 part c), the statement about $C\left(g_{1}\right) \mathcal{D}_{0}(B) \cap \cdots \cap C\left(g_{k}\right) \mathcal{D}_{0}(B)$ needs only to be checked in case $g_{1}=I_{2}$, since we may move the set by the automorphism defined by $g_{1}^{-1}$, which replaces $g_{1}, \ldots, g_{k}$ by $1, g_{1}^{-1} g_{2}, \ldots, g_{1}^{-1} g_{k}$. Thus, the considered sets will all be affine 
and it will simplify notations to work in the affine context. Assume $s(x \oplus y) s^{-1} \in \Xi_{m+n}$, where $s \in G L(m+n ; \mathbb{C}), x \in \mathfrak{M}_{m}(B), y \in \mathfrak{M}_{n}(B)$ and where $\Xi$ denotes one of the matricial sets we are considering. We must show there is $s^{\prime} \in G L(m ; \mathbb{C})$ such that $s^{\prime} x s^{\prime-1} \in \Xi_{m}$ (the statement is symmetric in $x$ and $y$, so only one half needs to be proved). Let $V=s\left(\mathbb{C}^{m} \oplus 0_{n}\right) \subset \mathbb{C}^{m+n}$ and assume $B$ has been identified with a $C^{*}$-algebra of operators on some Hilbert space $H$. Then $\mathfrak{M}_{n}(B)$ becomes a $C^{*}$-algebra of operators acting on $\mathbb{C}^{n} \otimes H$ and $V \otimes H$ is an invariant subspace for $s(x \oplus y) s^{-1}$ and $T=s(x \oplus y) s^{-1} \mid V \otimes H$ is an operator of norm $<1, \leq 1$ or unitary, depending on which of our first three possible $\Xi$ we consider (for the unitary case the restriction is isometric, but being similar to $x$ which is invertible, it must be unitary).

In the fourth case we already know $\|T\|<1$ and if $g_{j}^{-1}=\left(\begin{array}{ll}b_{1} & b_{2} \\ b_{3} & b_{4}\end{array}\right)$ any $2 \leq j \leq k$, then $I_{V} \otimes b_{1}+\left(I_{V} \otimes b_{2}\right) T$ is invertible because it is similar to $I_{m} \otimes b_{1}+\left(I_{m} \otimes b_{2}\right) x$ which is invertible and we have

$$
\begin{aligned}
& \left\|\left(I_{V} \otimes b_{3}+\left(I_{V} \otimes b_{4}\right) T\right)\left(I_{V} \otimes b_{1}+\left(I_{V} \otimes b_{2}\right) T\right)^{-1}\right\| \\
& \quad \leq\left\|\left(I_{n} \otimes b_{3}+\left(I_{n} \otimes b_{4}\right)\left(s(x \oplus y) s^{-1}\right)\right)\left(I_{n} \otimes b_{1}+\left(I_{n} \otimes b_{2}\right)\left(s(x \oplus y) s^{-1}\right)\right)^{-1}\right\|<1 .
\end{aligned}
$$

If $u: V \rightarrow \mathbb{C}^{m}$ is a unitary operator and if $s^{\prime}=u\left(V|s|_{\mathbb{C}^{m} \oplus 0_{n}}\right)$, then $s^{\prime} x s^{\prime-1}$ is unitarily equivalent to $\left.s(x \oplus y) s^{-1}\right|_{V \otimes H}$ and hence in $\Xi_{n}$.

An obvious characterization of $C(g) \mathcal{D}_{0}(B)_{n}$ which we used in the proof and which will be useful also in what follows, is that:

$$
\left(\begin{array}{ll}
\alpha & \beta \\
\gamma & \delta
\end{array}\right) / \widetilde{\lambda n} \in C(g) \mathcal{D}_{0}(B)_{n}
$$

where $g \in G L(2 ; B)$ and $g^{-1}=\left(\begin{array}{ll}b_{1} & b_{2} \\ b_{3} & b_{4}\end{array}\right)$ if and only if $\left(b_{1} \otimes I_{n}\right) \beta+$ $\left(b_{2} \otimes I_{n}\right) \delta$ is invertible and

$$
\left\|\left(\left(b_{3} \otimes I_{n}\right) \beta+\left(b_{4} \otimes I_{n}\right) \delta\right)\left(\left(b_{1} \otimes I_{n}\right) \beta+\left(b_{2} \otimes I_{n}\right) \delta\right)^{-1}\right\|<1 .
$$

Similarly $\left(\begin{array}{ll}\alpha & \beta \\ \gamma & \delta\end{array}\right) / \widetilde{\lambda n} \in C(g) \mathcal{U}(n ; B)$ iff $\left(b_{1} \otimes I_{n}\right) \beta+\left(b_{2} \otimes I_{n}\right) \delta$ is invertible and

$$
\left(\left(b_{3} \otimes I_{n}\right) \beta+\left(b_{4} \otimes I_{n}\right) \delta\right)\left(\left(b_{1} \otimes I_{n}\right) \beta+\left(b_{2} \otimes I_{n}\right) \delta\right)^{-1} \in \mathcal{U}(n ; B) .
$$

11.3. The stably matricial upper and lower half-planes $\left(\mathcal{H}_{ \pm}(B)\right)$. We will define some of the matricial sets which underly the noncommutative spectral analysis of a hermitian element with respect to $B$. This will be done via transformations $C(g)$ from $\mathcal{D}_{0}(B)$ and $\mathcal{U}(B)$. 
It will be convenient to have also a notation for the open stably matricial disk at infinity $\mathcal{D}_{\infty}(B)=C\left(\left(\begin{array}{ll}0 & 1 \\ 1 & 0\end{array}\right)\right) \mathcal{D}_{0}(B)$. The open upper and lower stably matricial half planes $\mathcal{H}_{+}(B)=\left(\mathcal{H}_{+}(B)_{n}\right)_{n \in \mathbb{N}}$ and $\mathcal{H}_{-}(B)=\left(\mathcal{H}_{-}(B)_{n}\right)_{n \in \mathbb{N}}$ are then defined by

$$
\begin{aligned}
& \mathcal{H}_{+}(B)=C\left(\left(\begin{array}{cc}
-i & i \\
1 & 1
\end{array}\right)\right) \mathcal{D}_{0}(B) \\
& \mathcal{H}_{-}(B)=C\left(\left(\begin{array}{cc}
i & -i \\
1 & 1
\end{array}\right)\right) \mathcal{D}_{0}(B)
\end{aligned}
$$

(which is just the familiar Cayley transforms in the notations we use). Equivalently we have

$$
\begin{aligned}
& \mathcal{H}_{+}(B)=C\left(\left(\begin{array}{cc}
i & -i \\
1 & 1
\end{array}\right)\right) \mathcal{D}_{\infty}(B) \\
& \mathcal{H}_{-}(B)=C\left(\left(\begin{array}{cc}
-i & i \\
1 & 1
\end{array}\right)\right) \mathcal{D}_{\infty}(B)
\end{aligned}
$$

and

$$
\mathcal{D}_{0}(B)=\left(\begin{array}{cc}
i & 1 \\
-i & 1
\end{array}\right) \mathcal{H}_{+}(B)=\left(\begin{array}{cc}
-i & 1 \\
i & 1
\end{array}\right) \mathcal{H}_{-}(B)
$$

Since $\mathcal{D}_{0}(B)^{*}=\mathcal{D}_{0}(B)$ we can use the formula

$$
(C(g) \pi)^{*}=C\left(W g^{*-1} W^{-1}\right) \pi^{*}
$$

where $W=\left(\begin{array}{cc}0 & 1 \\ -1 & 0\end{array}\right)$. This then easily gives

$$
\begin{aligned}
& \mathcal{D}_{\infty}(B)^{*}=\mathcal{D}_{\infty}(B) \text { and } \\
& \mathcal{H}_{+}(B)^{*}=\mathcal{H}_{-}(B) .
\end{aligned}
$$

The hermitian stably matricial set $\mathcal{H}(B)=\left(\mathcal{H}(B)_{n}\right)_{n \in \mathbb{N}}$ is obtained from $\mathcal{U}(B)$ by "Cayley transform"

$$
\mathcal{H}(B)=C\left(\left(\begin{array}{cc}
i & -i \\
1 & 1
\end{array}\right)\right) \mathcal{U}(B) .
$$

Since $\mathcal{U}(B)=\mathcal{U}(B)^{*}$ and $\mathcal{U}(B)=C\left(\left(\begin{array}{ll}0 & 1 \\ 1 & 0\end{array}\right)\right) \mathcal{U}(B)$, etc., it is easy to see that

$$
\mathcal{H}(B)=\mathcal{H}(B)^{*}
$$

and also

$$
\mathcal{H}(B)=C\left(\left(\begin{array}{cc}
-i & i \\
1 & 1
\end{array}\right)\right) \mathcal{U}(B)
$$


Remark. We have left out in the above discussion, because it seemed too well-known, the important fact that the affine part $\mathfrak{M}_{n}(B) \cap \mathcal{H}_{+}(B)_{n}$ of $\mathcal{H}_{+}(B)_{n}$ consists of those $\beta \in \mathfrak{M}_{n}(B)$ such that $\operatorname{Im} \beta \geq 0$ and $\operatorname{Im} \beta$ is invertible, that is $\operatorname{Im} \beta \geq \varepsilon I_{n} \otimes 1$ for some $\varepsilon>0$. To take this to the Grassmannian leads to Krein spaces (see [6]).

11.4. Mixed unit balls $\Delta_{p, q}(B)$ and half-planes $X_{p, q}(B)$ in the matricial resolvents of unitary and hermitian elements. Let $E$ be a unital $C^{*}$-algebra so that $1 \in B \subset E$ and let $u \in \mathcal{U}(1 ; E)$ and $\chi \in \mathcal{H}(E)_{1}$. We will show that in $\widetilde{\rho}(u ; B)$ and $\widetilde{\rho}(\chi ; B)$, the subsets $\mathcal{D}_{0}(B)_{n}, \mathcal{D}_{\infty}(B)_{n}, \mathcal{H}_{ \pm}(B)_{n}$ are part of some more general families of "unit balls" and "half-planes". Of course the questions for $\chi$ and $u$ are equivalent via Cayley transform.

If $x \in \mathcal{D}_{0}(B)_{n}$ then $x \in \widetilde{\rho}_{n}(u ; B)$ and $I_{n} \otimes u-x=\left(I_{n} \otimes u\right)\left(I_{n} \otimes 1-\left(I_{n} \otimes\right.\right.$ $\left.\left.u^{*}\right) x\right)$ is invertible because $\left\|\left(I_{n} \otimes u^{*}\right) x\right\|<1$. Since $C\left(\left(\begin{array}{ll}0 & 1 \\ 1 & 0\end{array}\right)\right) u=$ $u^{-1} \in \mathcal{U}(1 ; E)$ we also have $C\left(\left(\begin{array}{ll}0 & 1 \\ 1 & 0\end{array}\right)\right) \mathcal{D}_{0}(B)=\mathcal{D}_{\infty}(B) \subset \widetilde{\rho}(u ; B)$. Hence, $\widetilde{\rho}(u ; B)$ being fully matricial, if $x \in \mathcal{D}_{0}(B)_{p}, y \in \mathcal{D}_{0}(B)_{q}$ then $x \oplus C\left(\left(\begin{array}{ll}0 & 1 \\ 1 & 0\end{array}\right)\right) y \in \widetilde{\rho}_{p+q}(u ; B)$. Since $\widetilde{\rho}_{p+q}(u ; B)$ is open, but $\mathcal{D}_{0}(B)_{p} \oplus$ $C\left(\left(\begin{array}{ll}0 & 1 \\ 1 & 0\end{array}\right)\right) \mathcal{D}_{0}(B)_{q}$ isn't, if $p>0, q>0$, there should be a larger open set in $\widetilde{\rho}_{p+q}(u ; B)$ containing it. Such a set is $\Delta_{p, q}(B) \subset G r_{p+q}(B)$.

Using the action of $G L(2(p+q), \mathbb{C})$ on $G r_{p+q}(B)$ we define

$$
\Delta_{p, q}(B)=g_{p, q} \mathcal{D}_{0}(B)_{p+q}
$$

where

$$
g_{p, q}=\left(\begin{array}{cccc}
I_{p} & 0 & 0 & 0 \\
0 & 0 & 0 & I_{q} \\
0 & 0 & I_{p} & 0 \\
0 & I_{q} & 0 & 0
\end{array}\right) \in G L(2(p+q), \mathbb{C}) .
$$

In particular $\Delta_{p, 0}(B)=\mathcal{D}_{0}(B)_{p}$ and $\Delta_{0, q}(B)=\mathcal{D}_{\infty}(B)_{q}$.

From here we also define

$$
\begin{aligned}
\widetilde{\Delta}_{p, q}(B) & =G L(p+q ; \mathbb{C}) \cdot \Delta_{p, q}(B), \\
X_{p, q}(B) & =C\left(\left(\begin{array}{cc}
-i & i \\
1 & 1
\end{array}\right)\right) \Delta_{p, q}(B), \\
\widetilde{X}_{p, q}(B) & =G L(p+q ; \mathbb{C}) \cdot X_{p, q}(B) \\
& =C\left(\left(\begin{array}{cc}
-i & i \\
1 & 1
\end{array}\right)\right) \widetilde{\Delta}_{p, q}(B) .
\end{aligned}
$$


Since the definition of $\Delta_{p, q}(B)$ is tied to the special decomposition $\mathbb{C}^{p+q}=\mathbb{C}^{p} \oplus \mathbb{C}^{q}$, it is natural to consider also sets like

$$
\begin{aligned}
& \widehat{\Delta}_{p, q}(B)=U(p+q ; \mathbb{C}) \cdot \Delta_{p, q}(B) \\
& \widehat{X}_{p, q}(B)=U(p+q ; \mathbb{C}) \cdot X_{p, q}(B) .
\end{aligned}
$$

Clearly $X_{p, 0}(B)=\mathcal{H}_{+}(B)_{p}, \widetilde{X}_{p, 0}(B)=\widetilde{\mathcal{H}}_{+}(B)_{p}, X_{0, q}(B)=\mathcal{H}_{-}(B)_{q}$, $\widetilde{X}_{0, q}(B)=\widetilde{\mathcal{H}}_{-}(B)_{q}$.

Lemma. We have $\sigma \in \Delta_{p, q}(B)$ if and only if for some

$$
\left(\begin{array}{ll}
x & y \\
z & t
\end{array}\right) \in \mathcal{D}_{0}(B)_{p+q}
$$

we have

$$
\sigma=\left(\begin{array}{cccc}
0 & 0 & I_{p} \otimes 1 & 0 \\
0 & I_{q} \otimes 1 & z & t \\
I_{p} \otimes 1 & 0 & x & y \\
0 & 0 & 0 & I_{q} \otimes 1
\end{array}\right) / \widetilde{\lambda p+q}
$$

The proof is a straightforward computation which will be omitted.

Proposition. We have $\widetilde{\Delta}_{p, q}(B) \subset \widetilde{\rho}_{p+q}(u ; B)$ and $\widetilde{X}_{p, q}(B) \subset \widetilde{\rho}_{p+q}(x ; B)$. Moreover $C\left(\left(\begin{array}{ll}0 & 1 \\ 1 & 0\end{array}\right)\right) \widehat{\Delta}_{p, q}=\widehat{\Delta}_{q, p}, C\left(\left(\begin{array}{ll}0 & 1 \\ 1 & 0\end{array}\right)\right) \widetilde{\Delta}_{p, q}=\widetilde{\Delta}_{q, p}$ and $\widehat{X}_{p, q}^{*}=\widehat{X}_{q, p}, \widetilde{X}_{p, q}^{*}=\widetilde{X}_{q, p}$.

Proof. To establish the two inclusions it suffices to show $\Delta_{p, q}(B) \subset$ $\widetilde{\rho}_{p+q}(u ; B)$, since $\widetilde{\rho}(u ; B)$ is fully matricial and we can use Cayley transform to pass from $u$ to $\chi$. In view of the lemma, we must show that

$$
V=\left(\begin{array}{cccc}
I_{p} \otimes 1 & 0 & I_{p} \otimes 1 & 0 \\
z & t & 0 & I_{q} \otimes 1 \\
x & y & I_{p} \otimes u & 0 \\
0 & I_{q} \otimes 1 & 0 & I_{q} \otimes u
\end{array}\right)
$$

is invertible when

$$
\left(\begin{array}{ll}
x & y \\
z & t
\end{array}\right) \in \mathcal{D}_{0}(B)_{p+q}
$$

This is equivalent to the invertibility of

$$
g_{p, q} V\left(\begin{array}{cc}
I_{p+q+p} \otimes 1 & 0 \\
0 & I_{q} \otimes u^{-1}
\end{array}\right)=\left(\begin{array}{cccc}
I_{p} \otimes 1 & 0 & I_{p} \otimes 1 & 0 \\
0 & I_{q} \otimes 1 & 0 & I_{q} \otimes 1 \\
x & y & I_{p} \otimes u & 0 \\
z & t & 0 & I_{q} \otimes u^{-1}
\end{array}\right) .
$$


Multiplying to the right by

$$
\left(\begin{array}{cc}
I_{p+q} \otimes 1 & -I_{p+q} \otimes 1 \\
0 & I_{p+q} \otimes 1
\end{array}\right)
$$

we see that the invertibility of $V$ is equivalent to the invertibility of

$$
\begin{aligned}
& -\left(\begin{array}{cc}
x & y \\
z & t
\end{array}\right)+\left(\begin{array}{cc}
I_{p} \otimes u & 0 \\
0 & I_{q} \otimes u^{-1}
\end{array}\right) \\
& =\left(\begin{array}{cc}
I_{p} \otimes u & 0 \\
0 & I_{q} \otimes u^{-1}
\end{array}\right)\left(I_{p+q} \otimes 1-\left(\begin{array}{cc}
I_{p} \otimes u^{-1} & 0 \\
0 & I_{q} \otimes u
\end{array}\right)\left(\begin{array}{ll}
x & y \\
z & t
\end{array}\right)\right)
\end{aligned}
$$

which follows from the fact that

$$
\left\|\left(\begin{array}{cc}
I_{p} \otimes u^{-1} & 0 \\
0 & I_{q} \otimes u
\end{array}\right)\left(\begin{array}{ll}
x & y \\
z & t
\end{array}\right)\right\|<1 .
$$

Let $s_{p, q}=\left(\begin{array}{cc}0 & I_{p} \\ I_{q} & 0\end{array}\right) \in U(p+q ; \mathbb{C})$. The transformation of $\widehat{\Delta}_{p, q}$ and $\widetilde{\Delta}_{p, q}$ into $\widehat{\Delta}_{q, p}$ and $\widetilde{\Delta}_{q, p}$ via $C\left(\left(\begin{array}{ll}0 & 1 \\ 1 & 0\end{array}\right)\right)$ is a consequence of

$$
C\left(\left(\begin{array}{ll}
0 & 1 \\
1 & 0
\end{array}\right)\right) \Delta_{p, q}=s_{p, q} \cdot \Delta_{q, p}
$$

which in turn follows from the easy to check matrix equality

$$
\left(\begin{array}{cc}
0 & I_{p+q} \\
I_{p+q} & 0
\end{array}\right) g_{p, q}=\left(\begin{array}{cc}
s_{p, q} & 0 \\
0 & s_{p, q}
\end{array}\right) g_{q, p}\left(\begin{array}{cc}
s_{q, p} & 0 \\
0 & s_{q, p}
\end{array}\right) .
$$

Passing to the action of the involution on $\widetilde{X}_{p, q}, \widehat{X}_{p, q}$ we observe that if $\sigma \in G r_{n}(B)$ and $\gamma \in G L(2 n ; \mathbb{C})$ then $(\gamma \sigma)^{*}=\left(W_{n} \gamma^{*-1} W_{n}^{-1}\right) \sigma^{*}$ where $W_{n}=\left(\begin{array}{cc}0 & I_{n} \\ -I_{n} & 0\end{array}\right)$. If $\pi \in X_{p, q}$ then there is $\sigma \in \mathcal{D}_{0}(B)_{p+q}$ so that

$$
\pi=C_{p+q} g_{p, q} \sigma
$$

where $C_{p+q}$ stands for $\left(\begin{array}{cc}-i I_{p+q} & i I_{p+q} \\ I_{p+q} & I_{p+q}\end{array}\right)$. It is easily seen that $g_{p, q}^{*-1}=$ $g_{p, q}, C_{p+q}^{*-1}=\frac{1}{2} C_{p+q}$ and $W_{p+q} C_{p+q} W_{p+q}^{-1}=-i C_{p+q}\left(\begin{array}{cc}0 & I_{p+q} \\ I_{p+q} & 0\end{array}\right)$. Hence

$$
\pi^{*}=C_{p+q} \Gamma_{p+q} W_{p+q} g_{p, q} W_{p+q}^{-1} \sigma^{*}
$$

where $\Gamma_{n}=\left(\begin{array}{cc}0 & I_{n} \\ I_{n} & 0\end{array}\right)$. Let $d_{p, q}=\left(\begin{array}{cc}I_{p} & 0 \\ 0 & -I_{q}\end{array}\right) \in U(p+q ; \mathbb{C})$. It is easy to see that $W_{p+q} g_{p, q} W_{p+q}^{-1}=g_{p, q}\left(\begin{array}{cc}d_{p, q} & 0 \\ 0 & d_{p, q}\end{array}\right)$ so that

$$
\pi^{*}=C_{p+q} \Gamma_{p+q} g_{p, q}\left(d_{p, q} \cdot \sigma^{*}\right) .
$$


Since $d_{p, q} \sigma^{*} \in \mathcal{D}_{0}(B)_{p+q}$ we have

$$
\begin{aligned}
\pi^{*} \in C\left(\left(\begin{array}{cc}
-i & i \\
1 & 1
\end{array}\right)\right) C\left(\left(\begin{array}{ll}
0 & 1 \\
1 & 0
\end{array}\right)\right) \Delta_{p, q} & =C\left(\left(\begin{array}{cc}
-i & i \\
1 & 1
\end{array}\right)\right) s_{p, q} \cdot \Delta_{q, p} \\
& =s_{p, q} \cdot X_{q, p} .
\end{aligned}
$$

Thus we have proved

This easily implies

$$
X_{p, q}^{*} \subset s_{p, q} X_{q, p}
$$

$$
\begin{aligned}
& \widehat{X}_{p, q}^{*} \subset \widehat{X}_{q, p} \text { and } \\
& \widetilde{X}_{p, q}^{*} \subset \widetilde{X}_{q, p}
\end{aligned}
$$

which by symmetry must be equalities.

11.5. Extending the invertibility of stably matricial functions. Inverse function theorems usually provide local inverses and this raises the question whether we can use them to get fully matricial inverses. We prove here the results on stably matricial functions which make such uses of inverse function theorems possible.

Lemma. Let $\Xi$ be an open stably matricial $B$-set and let $f=\left(f_{n}\right)_{n \in \mathbb{N}}$ be an $\mathcal{X}$-valued continuous stably matricial function on $\Xi(\mathcal{X}$ a Banach space). If $f$ is injective then also its fully matricial extension $\widetilde{f}$ to $\widetilde{\Xi}$ is injective.

Proof. Assume $\pi, \pi^{\prime} \in \Xi_{n}$ and $s, s^{\prime} \in G L(n ; \mathbb{C})$ are such that $\tilde{f}_{n}(s \cdot \pi)=$ $\tilde{f}_{n}\left(s^{\prime} \cdot \pi^{\prime}\right)$. Let $s^{\prime \prime}=\left(\begin{array}{cc}0 & s^{-1} s^{\prime} \\ s^{\prime-1} s & 0\end{array}\right) \in G L(2 n ; \mathbb{C})$ and let $\pi^{\prime \prime}=\pi \oplus \pi^{\prime} \in$ $\Xi_{2 n}$. We have $\widetilde{f}_{2 n}\left(s^{\prime \prime} \cdot \pi^{\prime \prime}\right)=\widetilde{f}_{2 n}\left(\pi^{\prime \prime}\right)$ or equivalently $\left(\operatorname{Ad} s^{\prime \prime}\right) f_{2 n}\left(\pi^{\prime \prime}\right)=$ $f_{2 n}\left(\pi^{\prime \prime}\right)$. This implies that the matrix $f_{2 n}\left(\pi^{\prime \prime}\right)$ with entries in $\mathcal{X}$ commutes with the scalar matrix $s^{\prime \prime}$. It is easily seen that this implies $f_{2 n}\left(\pi^{\prime \prime}\right)$ commutes with any matrix $\sigma$ in the algebra generated by $I_{2 n}$ and $s^{\prime \prime}$. We may then choose $\sigma$ to be a logarithm of $s^{\prime \prime}$. We will then have

$$
(\operatorname{Ad}(\exp t \sigma)) f_{2 n}\left(\pi^{\prime \prime}\right)=f_{2 n}\left(\pi^{\prime \prime}\right)
$$

for all $t \in \mathbb{C}$.

Since $\Xi$ is open, there is $\varepsilon>0$ such that $(\exp t \sigma) \cdot \pi^{\prime \prime} \in \Xi$ if $|t|<\varepsilon$. Since $f$ is injective we infer that $(\exp t \sigma) \cdot \pi^{\prime \prime}=\pi^{\prime \prime}$ if $|t|<\varepsilon$. If $m \in \mathbb{N}$ is such that $m>\varepsilon^{-1}$ then we have $s^{\prime \prime} \cdot \pi^{\prime \prime}=\left(\exp m^{-1} \sigma\right) \cdot \pi^{\prime \prime}=\pi^{\prime \prime}$. This in turn implies that $s^{-1} s^{\prime} \cdot \pi^{\prime}=\pi$ and hence we have $s \cdot \pi=s^{\prime} \cdot \pi^{\prime}$.

Proposition. Let $\Xi$ be an affine open stably matricial B-set and let $f$ be a B-valued injective stably matricial function on $\Xi$. Assume $f$ is 
continuous and open. Then $\widetilde{f}(\widetilde{\Xi})=\left(\widetilde{f}_{n}\left(\widetilde{\Xi}_{n}\right)\right)_{n \in \mathbb{N}}$ is an open fully matricial B-set and each map $\widetilde{f}_{n}$ is a homeomorphism of $\widetilde{\Xi}_{n}$ onto $\widetilde{f}_{n}\left(\widetilde{\Xi}_{n}\right)$. Moreover the inverse map $\widetilde{f}^{-1}=\left(\widetilde{f}_{n}^{-1}\right)_{n \in \mathbb{N}}$ is fully matricial. If additionally $f$ is analytic, then so is $\tilde{f}^{-1}$.

Proof. The lemma guarantees that each $\widetilde{f}_{n}$ is a bijection of $\widetilde{\Xi}_{n}$ onto $\widetilde{f}_{n}\left(\widetilde{\Xi}_{n}\right)$. Since $f$ is continuous and open and $\widetilde{f}_{n}$ is $G L(n ; \mathbb{C})$-equivariant it follows $\widetilde{f}_{n}$ is a homeomorphism onto an open set. Also since $\widetilde{f}$ preserves the structures involved (direct sum, equivariance, topology) its inverse is also fully matricial. In the analytic case $\widetilde{f}^{-1}$ is clearly analytic.

A typical application of the preceding proposition would run as follows. If $\mathcal{X}$ is a Banach space and $f:\{x \in \mathcal{X} \mid\|x\|<R\} \rightarrow \mathcal{X}$, $f(0)=0$, is a holomorphic map so that $\|f(x)\| \leq C$ when $\|x\|<R$ and $\left\|(D f)(0)^{-1}\right\|=M<\infty$, then the usual inverse function results give that there exist $\varepsilon_{1}>0$ and $\varepsilon_{2}>0$ which depend only on $R^{-1} C M$ such that the restriction of $f$ to $\left\{x \in \mathcal{X} \mid\|x\|<\varepsilon_{1} R\right\}$ is an analytic isomorphism onto an open subset of $\mathcal{X}$ which contains $\left\{x \in \mathcal{X} \mid\|x\|<\varepsilon_{2} R M^{-1}\right\}$. (Replacing $f$ by $R^{-1}((D f)(0))^{-1} \circ f(R \cdot)$ this reduces to the case $R=1$ and $(D f)(0)=I$.) Assuming $B$ is a $C^{*}$-algebra (for control of matricial norms) and remarking that the differential of a stably matricial $B$-valued function in $R \mathcal{D}_{0}(B)$ has as components at the origin the multiples of the differential of the first component, we can apply the inverse function result to the components of the function and combine this with the Proposition to get the following corollary.

Corollary. Assume $B$ is a $C^{*}$-algebra and assume $f$ is a B-valued analytic stably matricial map on $R \mathcal{D}_{0}(B)$ such that $f_{1}(0)=0$ and $f\left(R \mathcal{D}_{0}(B)\right) \subset C \mathcal{D}_{0}(B)$. Suppose $\left(D f_{1}\right)(0)^{-1}$ exists and $\left\|\left(D f_{1}\right)(0)^{-1}\right\|<$ $M$, then there are $\varepsilon_{1}>0, \varepsilon_{2}>0$ which depend only on $R^{-1} C M$ so that $\tilde{f}$ is a fully matricial isomorphism of $\varepsilon_{1} R \widehat{\mathcal{D}_{0}(B)}$ onto an open fully matricial affine $B$-set which contains $\varepsilon_{2} R M^{-1} \widehat{\mathcal{D}_{0}(B)}$. In particular the inverse of $\tilde{f}$ is defined on a fully matricial set containing $\varepsilon_{2} R M^{-1} \widetilde{\mathcal{D}_{0}(B)}$ and is fully matricial holomorphic.

\section{More ABOUT DUALITY TRANSFORMS FOR UNITARY AND HERMITIAN ELEMENTS OF THE GRASSMANNIAN}


12.1. Universal duality transforms for unitary and hermitian elements. The universal duality transforms which we construct in this subsection are the duality transforms for some universal unitary and hermitian Grassmannian elements relative to a given unital $C^{*}$-algebra $B$. We will then prove in 12.2-12.5 that certain technical assumptions we made in connection with the duality transform in 6.4 and in 8.3 are fulfilled in the universal case (8.3 only for the unitary case).

Let $E(B)=B * C(\mathbb{T})$ be the unital full free product $C^{*}$-algebra (that is, the $C^{*}$-algebra free product with amalgamation over $\mathbb{C} 1$ ) of $B$ and $C(\mathbb{T})$. We shall identify $B$ and $C(\mathbb{T})$ with the corresponding $C^{*}$-subalgebras of $E(B)$. Identifying $\mathbb{T}$ with the unit circle in $\mathbb{C}$, the function $\mathbb{T} \rightarrow \mathbb{C}$ giving the embedding, is then a unitary element $u \in$ $E(B)$, so that $C(\mathbb{T}) \ni f \rightarrow f(u) \in E(B)$ is the inclusion of $C(\mathbb{T})$ into $E$. We shall also denote by $u$ the element in $G r_{1}(E(B))$ corresponding to $u$, that is $\left(\begin{array}{ll}0 & 1 \\ 1 & u\end{array}\right) / \widetilde{\lambda 1}$. The universal hermitian element is then $\chi=C\left(\left(\begin{array}{cc}-i & i \\ 1 & 1\end{array}\right)\right) u=\left(\begin{array}{cc}i & i(u-1) \\ 1 & u+1\end{array}\right) / \widetilde{\lambda 1} \in G r_{1}(E(B))$. If $E$ is a unital $C^{*}$-algebra with a given unitary element $v$ and containing $B$ as a unital $C^{*}$-subalgebra, then there is a unique unital $*$-homomorphism $j: E(B) \rightarrow E$ acting as the identity on $B$ and so that $j(u)=v$. Then the duality transform $\mathcal{U}_{v}: E^{d} \rightarrow \mathcal{A}(\widetilde{\rho}(v ; B))$ is so that $\widetilde{\rho}(u ; B) \subset \widetilde{\rho}(v ; B)$ and $\left.\mathcal{U}_{v}(\varphi)\right|_{\widetilde{\rho}(v ; B)}=\mathcal{U}_{u}(\varphi \circ j)$. Along the same lines, in the hermitian case, if $E$ is now a unital $C^{*}$-algebra containing $B$ and $h \in G r_{1}(E)$ is a hermitian element, then there is $v \in E$ a unitary element so that $h=C\left(\left(\begin{array}{cc}-i & i \\ 1 & 1\end{array}\right)\right) v$ and there is $j: E(B) \rightarrow E,\left.j\right|_{B}=\operatorname{id}_{B}, j(u)=v$. Then $\left.\mathcal{U}_{h}(\varphi)\right|_{\tilde{\rho}(\chi ; B)}=\mathcal{U}_{\chi}(\varphi \circ j)$.

Note that several universal objects arise from working in $E(B)$ with $u$ and $\chi$. The resolvent sets $\widetilde{\rho}(u ; B), \widetilde{\rho}(\chi ; B)$ are universal, in the sense that any resolvents $\widetilde{\rho}(v ; B)$ and $\widetilde{\rho}(h ; B)$ as above contain these. Also the algebras $\mathcal{L R}(u ; B)$ and $\mathcal{L R}(\chi ; B)$ are universal as they map into $\mathcal{L R}(v ; B)$ 's and $\mathcal{L R}(h ; B)$ 's respectively.

12.2. A strengthened assumption 6.4 is satisfied by the universal unitary element. We shall prove assumption 6.4 for the universal unitary element $u \in E(B)$ in a stronger form where $\mathcal{L R}(u ; B)$ is replaced by the larger algebra $\mathcal{Q R}(u ; B)$ which is the inverse closed subalgebra of $E(B)$ generated by $B \cup \mathcal{L R}(u ; B)$. Note that since $0 \in \rho_{1}(u ; B)$ we have $u^{-1} \in \mathcal{L} \mathcal{R}(u ; B)$ and hence $B\langle u\rangle \subset \mathcal{Q R}(u ; B)$.

That assumption 6.4 in its original form, holds for the universal unitary element, will then be obtained as a corollary. 
Proposition. There is a derivation

$$
\partial_{u: B}^{\mathcal{Q}}: \mathcal{Q R}(u ; B) \rightarrow \mathcal{Q R}(u ; B) \otimes \mathcal{Q R}(u ; B)
$$

such that

$$
\left(\operatorname{id}_{\mathfrak{M}_{n}} \otimes \partial_{u: b}^{\mathcal{Q}}\right) \widetilde{\mathcal{R}}_{n}(u ; B)(\sigma)=\widetilde{\mathcal{R}}_{n}(u ; B)(\sigma) \otimes_{\mathfrak{M}_{n}} \widetilde{\mathcal{R}}_{n}(u ; B)(\sigma)
$$

for all $n \in \mathbb{N}$ and $\sigma \in \widetilde{\rho}_{n}(u ; B)$. Moreover we have

$$
\left.\partial_{u: b}^{\mathcal{Q}}\right|_{B}=0 \text { and } \partial_{u: B}^{\mathcal{Q}} u=1 \otimes 1
$$

Proof. Let $\mu$ be a faithful representation of $E(B)$ on some Hilbert space $\mathcal{H}$. The universal property of the full free product implies that for any fixed $H=H^{*} \in \mathcal{B}(\mathcal{H})$ and all $\varepsilon \in \mathbb{R}$ there are representations $\mu_{\varepsilon}: E(B) \rightarrow \mathcal{B}(\mathcal{H})$ so that $\mu_{\varepsilon}|B=\mu| B$ and $\mu_{\varepsilon}(u)=U U(\varepsilon)$ where $U=\mu(u)$ and $U(\varepsilon)=\exp (i \varepsilon H)$. It will be convenient to identify $B$ with $\mu(B)$ (that is, to assume $B$ is a $C^{*}$-subalgebra of $\mathcal{B}(\mathcal{H})$ ). If $\sigma=\left(\begin{array}{ll}\alpha & \beta \\ \gamma & \delta\end{array}\right) / \widetilde{\lambda n} \in \widetilde{\rho}_{n}(u ; B)$, then $\left(\delta-\left(I_{n} \otimes U U(\varepsilon)\right) \beta\right)^{-1}$ exists and

$$
\left(\operatorname{id}_{\mathfrak{M}_{n}} \otimes \mu_{\varepsilon}\right)\left(\widetilde{\mathcal{R}}_{n}(u ; B)(\sigma)\right)=\beta\left(\delta-\left(I_{n} \otimes U U(\varepsilon)\right) \beta\right)^{-1} .
$$

It follows that $\mu_{\varepsilon}(x)$ is differentiable at $\varepsilon=0$, as a function of $\varepsilon \in \mathbb{R}$, when $x \in \mathcal{L} \mathcal{R}(u ; B)$ and since $\mu_{\varepsilon}(b)$ is constant for $b \in B$, differentiability actually holds for $x \in B \cup \mathcal{L R}(u ; B)$ and hence for $x \in \mathcal{Q R}(u ; B)$. This yields a derivation

$$
d_{H}: \mathcal{Q R}(u ; B) \rightarrow \mathcal{B}(\mathcal{H})
$$

with respect to the $\mathcal{Q R}(u ; B)$-bimodule structure on $\mathcal{B}(\mathcal{H})$ defined by $\mu$, where

We then have

$$
d_{H}(x)=-\left.i \frac{d}{d \varepsilon} \mu_{\varepsilon}(x)\right|_{\varepsilon=0} .
$$

$$
\begin{aligned}
& \left(\operatorname{id}_{\mathfrak{M}_{n}} \otimes d_{H}\right)\left(\widetilde{\mathcal{R}}_{n}(u ; B)(\sigma)\right)=\widetilde{\mathcal{R}}_{n}(U ; B)(\sigma)\left(I_{n} \otimes U H\right) \widetilde{\mathcal{R}}_{n}(U ; B)(\sigma) \\
& =\left(\operatorname{id}_{\mathfrak{M}_{n}} \otimes \mu\right)\left(\widetilde{\mathcal{R}}_{n}(u ; B)(\sigma)\right)\left(I_{n} \otimes U H\right)\left(\operatorname{id}_{\mathfrak{M}_{n}} \otimes \mu\right)\left(\widetilde{\mathcal{R}}_{n}(u ; B)(\sigma)\right)
\end{aligned}
$$

and moreover

$$
\left.d_{H}\right|_{B}=0 \text { and } d_{H} u=U H
$$

We may extend by complex linearity the map $H \rightsquigarrow d_{H}$ to $\mathcal{B}(\mathcal{H})$ and get derivations $d_{T}: \mathcal{Q R}(u ; B) \rightarrow \mathcal{B}(\mathcal{H})$ with $T \in \mathcal{B}(\mathcal{H})$. Putting these together yields a derivation

$$
d: \mathcal{Q R}(u ; B) \rightarrow \mathcal{B}(\mathcal{H})^{\mathcal{B}(\mathcal{H})}
$$


where $d(x)=\left(d_{T}(x)\right)_{T \in \mathcal{B}(\mathcal{H})}$ and the $\mathcal{Q R}(u ; B)$-bimodule structure on $\mathcal{B}(\mathcal{H})^{\mathcal{B}(\mathcal{H})}$ is deduced from $\mu^{\mathcal{B}(\mathcal{H})}$. We also have a $\mathcal{Q R}(u ; B)$-bimodules map

where

$$
\varphi: \mathcal{Q R}(u ; B) \otimes \mathcal{Q R}(u ; B) \rightarrow \mathcal{B}(\mathcal{H})^{\mathcal{B}(\mathcal{H})}
$$

$$
\varphi(x \otimes y)=(\mu(x) U T \mu(y))_{T \in \mathcal{B}(\mathcal{H})} .
$$

It is easily seen that $\varphi$ is injective and that we have

$$
\begin{aligned}
\left(\operatorname{id}_{\mathfrak{M}_{n}} \otimes d\right)\left(\widetilde{\mathcal{R}}_{n}(u ; B)(\sigma)\right) & =\left(\operatorname{id}_{\mathfrak{M}_{n}} \otimes \varphi\right)\left(\widetilde{\mathcal{R}}_{n}(u ; B)(\sigma) \otimes_{\mathfrak{M}_{n}} \widetilde{\mathcal{R}}_{n}(u ; B)(\sigma)\right), \\
\left.d\right|_{B} & =0 \text { and } d(u)=\varphi(1 \otimes 1) .
\end{aligned}
$$

The formula for $\left(\operatorname{id}_{\mathfrak{M}_{n}} \otimes d\right)\left(\widetilde{\mathcal{R}}_{n}(u ; B)(\sigma)\right)$ implies that

$$
d(\mathcal{L} \mathcal{R}(u ; B)) \subset \varphi(\mathcal{L} \mathcal{R}(u ; B) \otimes \mathcal{L} \mathcal{R}(u ; B)) .
$$

The set

$$
\{x \in \mathcal{Q R}(u ; B) \mid d(x) \in \varphi(\mathcal{Q R}(u ; B) \otimes \mathcal{Q R}(u ; B))\}
$$

is an inverse-closed subalgebra of $\mathcal{Q R}(u ; B)$ and since it contains $\mathcal{L} \mathcal{R}(u ; B)$ and $B$ it is equal to $\mathcal{Q R}(u ; B)$. From

$$
d(\mathcal{Q R}(u ; B)) \subset \varphi(\mathcal{Q R}(u ; B) \otimes \mathcal{Q R}(u ; B))
$$

we infer the existence of a linear map $\partial_{u: B}^{\mathcal{Q}}: \mathcal{Q R}(u ; B) \rightarrow \mathcal{Q R}(u ; B) \otimes$ $\mathcal{Q R}(u ; B)$, such that $\varphi \circ \partial_{u: B}^{\mathcal{Q}}=d$. Since $d$ is a derivation we easily infer that $\partial_{u: B}^{\mathcal{Q}}$ is also a derivation and the properties of $\partial_{u: B}^{\mathcal{Q}}$ are easily obtained from those of $d$.

Corollary. There is a derivation

$$
\partial_{u: B}: \mathcal{L} \mathcal{R}(u ; B) \rightarrow \mathcal{L} \mathcal{R}(u ; B) \otimes \mathcal{L} \mathcal{R}(u ; B)
$$

such that

$$
\left(\operatorname{id}_{\mathfrak{M}_{n}} \otimes \partial_{u: B}\right) \widetilde{\mathcal{R}}_{n}(u ; B)(\sigma)=\widetilde{\mathcal{R}}_{n}(u ; B)(\sigma) \otimes_{\mathfrak{M}_{n}} \widetilde{\mathcal{R}}_{n}(u ; B)(\sigma)
$$

for all $n \in \mathbb{N}$ and $\sigma \in \widetilde{\rho}_{n}(u ; B)$.

Proof. We can take

$$
\partial_{u: B}=\partial_{u: B}^{\mathcal{Q}} \mid \mathcal{L} \mathcal{R}(u ; B)
$$

and remark, like we did in the proof of the Proposition that the formula for $\left.\left(\operatorname{id}_{\mathfrak{M}_{n}} \otimes \partial_{u: b}^{\mathcal{Q}}\right)\left(\widetilde{\mathcal{R}}_{n}(u ; B)\right)(\sigma)\right)$ implies that

$$
\partial_{u: B}^{\mathcal{Q}}(\mathcal{L} \mathcal{R}(u ; B)) \subset \mathcal{L} \mathcal{R}(u ; B) \otimes \mathcal{L} \mathcal{R}(u ; B) .
$$

Remark. It is easy to see that the larger algebra $\mathfrak{M} \mathcal{Q R}(u ; B) \supset$ $\mathcal{Q R}(u ; B)$, which is the closure under taking entries of the inverses of 
invertible square matrices with entries in the algebra, is still contained in the domain of definition of the derivations $d_{H}$. This yields a proof of the modified Proposition with $\mathcal{Q R}(u ; B)$ replaced by $\mathfrak{M} \mathcal{Q R}(u ; B)$ and $\partial_{u: B}^{\mathcal{Q}}$ by a derivation

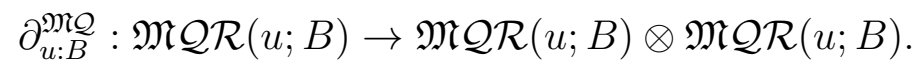

12.3. Assumption 8.3 is satisfied by the universal unitary element. We shall prove the following result (assumption 8.3) about the universal unitary element $u \in E(B)$.

Proposition. There is a linear map $D: \mathcal{L R}(u ; B) \rightarrow E(B)$ so that

$$
\left(\operatorname{id}_{\mathfrak{M}_{n}} \otimes D\right)\left(\widetilde{\mathcal{R}}_{n}(u ; B)(\sigma)\right)=\left.\frac{d}{d t} \widetilde{\mathcal{R}}_{n}\left(e^{t} u ; B\right)(\sigma)\right|_{t=0}
$$

for all $n \in \mathbb{N}$ and $\sigma \in \widetilde{\rho}_{n}(u ; B)$.

Proof. Since $E(B)$ is a free product of $B$ and $C(\mathbb{T})$, there is a one-parameter group of automorphisms $\alpha(t), t \in \mathbb{R}$ of $E(B)$ such that $\left.\alpha(t)\right|_{B}=\operatorname{id}_{B}$ and $\alpha(t)(u)=e^{i t} u$. It follows that $\widetilde{\rho}_{n}(u ; B)=$ $\widetilde{\rho}_{n}(\alpha(t) u ; B)$.

On the other hand it is easily seen that $\widetilde{\mathcal{R}}_{n}\left(e^{t} u ; B\right)$ is holomorphic in $t$ in a neighborhood of zero. This gives

$$
\begin{aligned}
\left.\frac{d}{d t} \widetilde{\mathcal{R}}_{n}\left(e^{t} u ; B\right)(\sigma)\right|_{t=0} & =-\left.i \frac{d}{d t} \widetilde{\mathcal{R}}_{n}(\alpha(t) u ; B)(\sigma)\right|_{t=0} \\
& =-\left.i \frac{d}{d t}\left(\operatorname{id}_{\mathfrak{M}_{n}} \otimes \alpha(t)\right) \widetilde{\mathcal{R}}_{n}(u ; B)(\sigma)\right|_{t=0}
\end{aligned}
$$

taking into account that the automorphism of the Grassmannian induced by $\alpha(t)$ leaves $\sigma$ fixed since it lies in $\operatorname{Gr}_{n}(B)$.

By the definition of $\widetilde{\mathcal{R}}_{n}(u ; B)(\sigma)$, since $I_{n} \otimes u$ is in the domain of definition of the infinitesimal generator of $\operatorname{id}_{\mathfrak{M}_{n}} \otimes \alpha(t)$ we infer that $\widetilde{\mathcal{R}}_{n}(u ; B)(\sigma)$ is also in the domain of definition of the infinitesimal generator of $\operatorname{id}_{\mathfrak{M}_{n}} \otimes \alpha(t)$. This in turn gives that the matrix-coefficients of $\widetilde{\mathcal{R}}_{n}(u ; B)(\sigma)$ are in the domain of definition of the infinitesimal generator $P=\left.\frac{d}{d t} \alpha(t)\right|_{t=0}$ of $\alpha(t)$. Thus $\mathcal{L} \mathcal{R}(u ; B)$ is in the domain of definition of $P$ and we can define $D$ to be $-i P$.

12.4. The relation of $\mathcal{L} \mathcal{R}(\chi ; B)$ and $\widetilde{\mathcal{R}}_{n}(\chi ; B)$ to $\mathcal{Q R}(u ; B), \widetilde{\mathcal{R}}_{n}(u ; B)$ and $\widetilde{\mathcal{R}}_{n}\left(u^{-1} ; B\right)$. Since $\chi$ was defined as a "fractional-linear transform" of $u$ certain results about $\chi$ can be derived from results about $u$. We collect in this section some technical facts underlying the passage from $u$ to $\chi$. 
Since $\chi=C\left(\left(\begin{array}{cc}-i & i \\ 1 & 1\end{array}\right)\right) u$ it is immediate that $\widetilde{\rho}_{n}(\chi ; B)=C_{n}\left(\left(\begin{array}{cc}-i & i \\ 1 & 1\end{array}\right)\right) \widetilde{\rho}_{n}(u ; B)$.

Also, since there is an automorphism $\gamma$ of $E(B)$ such that $\gamma \mid B=\mathrm{id}_{B}$ and $\gamma(u)=u^{-1}$, we have $\widetilde{\rho}_{n}(u ; B)=\widetilde{\rho}_{n}\left(u^{-1} ; B\right)$. On the other hand $C\left(\left(\begin{array}{ll}0 & 1 \\ 1 & 0\end{array}\right)\right) u=u^{-1}$ and hence $C_{n}\left(\left(\begin{array}{ll}0 & 1 \\ 1 & 0\end{array}\right)\right) \widetilde{\rho}_{n}(u ; B)=\widetilde{\rho}_{n}\left(u^{-1} ; B\right)$ and $C_{n}\left(\left(\begin{array}{ll}0 & 1 \\ 1 & 0\end{array}\right)\right) \widetilde{\rho}_{n}(u ; B)=\widetilde{\rho}_{n}(u ; B)$.

Lemma. Let $\sigma=\left(\begin{array}{ll}\alpha & \beta \\ \gamma & \delta\end{array}\right) / \widetilde{\lambda n} \in \widetilde{\rho}_{n}(u ; B), \sigma^{-1}=C_{n}\left(\left(\begin{array}{ll}0 & 1 \\ 1 & 0\end{array}\right)\right) \sigma=$ $\left(\begin{array}{ll}\gamma & \delta \\ \alpha & \beta\end{array}\right) / \widetilde{\lambda n}$ and let $\nu=C_{n}\left(\left(\begin{array}{cc}-i & i \\ 1 & 1\end{array}\right)\right) \sigma \in \widetilde{\rho}_{n}(\chi ; B)$. We have:

a) $\left(I_{n} \otimes u\right)\left(\widetilde{\mathcal{R}}_{n}(u ; B)(\sigma)\right)\left(I_{n} \otimes u\right)+I_{n} \otimes u=-\widetilde{\mathcal{R}}_{n}\left(u^{-1} ; B\right)\left(\sigma^{-1}\right)$

b) $\mathcal{L} \mathcal{R}\left(u^{-1} ; B\right) \subset \mathcal{Q R}(u ; B)$

c) $\widetilde{\mathcal{R}}_{n}(\chi ; B)(\nu)=-\frac{i}{2} \widetilde{\mathcal{R}}_{n}\left(u^{-1} ; B\right)\left(\sigma^{-1}\right)\left(I_{n} \otimes 1-I_{n} \otimes u^{-1}\right)$

$+\frac{i}{2} \widetilde{\mathcal{R}}_{n}(u ; B)(\sigma)\left(I_{n} \otimes 1-I_{n} \otimes u\right)$

d) $\mathcal{L} \mathcal{R}(\chi ; B) \subset \mathcal{Q R}(u ; B)$.

Proof. a) implies b), since $\{u\} \cup \mathcal{L} \mathcal{R}(u ; B) \subset \mathcal{Q R}(u ; B)$.

On the other hand, a) is equivalent to

$$
\left(I_{n} \otimes u\right) \widetilde{\mathcal{R}}_{n}(u ; B)(\sigma)+\widetilde{\mathcal{R}}_{n}\left(u^{-1} ; B\right)\left(\sigma^{-1}\right)\left(I_{n} \otimes u^{-1}\right)=-I_{n} \otimes 1 .
$$

This follows from the computation of the left hand side, which is equal to

$$
\begin{aligned}
& \left(I_{n} \otimes u\right) \beta\left(\delta-\left(I_{n} \otimes u\right) \beta\right)^{-1}+\delta\left(\beta-\left(I_{n} \otimes u^{-1}\right) \delta\right)^{-1}\left(I_{n} \otimes u^{-1}\right) \\
& =\left(I_{n} \otimes u\right) \beta\left(\delta-\left(I_{n} \otimes u\right) \beta\right)^{-1}+\delta\left(\left(I_{n} \otimes u\right) \beta-\delta\right)^{-1} \\
& =\left(\left(I_{n} \otimes u\right) \beta-\delta\right)\left(\delta-\left(I_{n} \otimes u\right) \beta\right)^{-1}=-I_{n} \otimes 1 .
\end{aligned}
$$

Since

$$
\left\{u, u^{-1}\right\} \cup \mathcal{L R}(u ; B) \cup \mathcal{L} \mathcal{R}\left(u^{-1} ; B\right) \subset \mathcal{Q R}(u ; B)
$$

we see that $d$ ) is a consequence of c). So, the only thing we still must prove is $\mathrm{c}$ ).

In view of the definition of $\chi$ and $\nu$, to compute $\widetilde{\mathcal{R}}_{n}(\chi ; B)(\sigma)$ we must first compute the 22-entry of the block-matrix

$$
\begin{aligned}
& \left(\left(\begin{array}{cc}
-i I_{n} \otimes 1 & i I_{n} \otimes 1 \\
I_{n} \otimes 1 & I_{n} \otimes 1
\end{array}\right)\left(\begin{array}{cc}
I_{n} \otimes 1 & \beta \\
I_{n} \otimes u & \delta
\end{array}\right)\right)^{-1} \\
& =\frac{1}{2}\left(\left(\begin{array}{cc}
I_{n} \otimes 1 & O \\
I_{n} \otimes u & \delta-\left(I_{n} \otimes u\right) \beta
\end{array}\right)\left(\begin{array}{cc}
I_{n} \otimes 1 & \beta \\
O & I_{n} \otimes 1
\end{array}\right)\right)^{-1}\left(\begin{array}{cc}
i I_{n} \otimes 1 & I_{n} \otimes 1 \\
-i I_{n} \otimes 1 & I_{n} \otimes 1
\end{array}\right)
\end{aligned}
$$




$$
\begin{aligned}
& =\frac{1}{2}\left(\begin{array}{cc}
I_{n} \otimes 1 & -\beta \\
O & I_{n} \otimes 1
\end{array}\right)\left(\begin{array}{cc}
O \\
-\left(\delta-\left(I_{n} \otimes u\right) \beta\right)^{-1}\left(I_{n} \otimes u\right) & \left(\delta-\left(I_{n} \otimes u\right) \beta\right)^{-1}
\end{array}\right)\left(\begin{array}{cc}
i I_{n} \otimes 1 & I_{n} \otimes 1 \\
-i I_{n} \otimes 1 & I_{n} \otimes 1
\end{array}\right) \\
& =\left(\begin{array}{cc}
* & O \\
-\frac{1}{2}\left(\delta-\left(I_{n} \otimes u\right) \beta\right)^{-1}\left(I_{n} \otimes u\right) & \frac{1}{2}\left(\delta-\left(I_{n} \otimes u\right) \beta\right)^{-1}
\end{array}\right)\left(\begin{array}{cc}
i I_{n} \otimes 1 & I_{n} \otimes 1 \\
-i I_{n} \otimes 1 & I_{n} \otimes 1
\end{array}\right) \\
& =\left(\begin{array}{cc}
* & * \\
* & \frac{1}{2}\left(\delta-\left(I_{n} \otimes u\right) \beta\right)^{-1}\left(I_{n} \otimes(1-u)\right)
\end{array}\right) .
\end{aligned}
$$

To get $\widetilde{\mathcal{R}}_{n}(\chi ; B)(\nu)$, we must multiply this entry to the left by the 12-entry of $\nu$, which is $i(\beta-\delta)$. Thus we have

$$
\begin{aligned}
& \widetilde{\mathcal{R}}_{n}(\chi ; B)(\nu)=\frac{i}{2}(\beta-\delta)\left(\delta-\left(I_{n} \otimes u\right) \beta\right)^{-1}\left(I_{n} \otimes(1-u)\right) \\
& =\frac{i}{2}\left(\widetilde{\mathcal{R}}_{n}(u ; B)(\sigma)\right)\left(I_{n} \otimes(1-u)\right)-\frac{i}{2} \delta\left(\left(I_{n} \otimes u^{-1}\right) \delta-\beta\right)^{-1}\left(I_{n} \otimes u^{-1}\right)\left(I_{n} \otimes(1-u)\right) \\
& =\frac{i}{2}\left(\widetilde{\mathcal{R}}_{n}(u ; B)(\sigma)\right)\left(I_{n} \otimes(1-u)\right)-\frac{i}{2}\left(\widetilde{\mathcal{R}}_{n}\left(u^{-1} ; B\right)\left(\sigma^{-1}\right)\right)\left(I_{n} \otimes\left(1-u^{-1}\right)\right) .
\end{aligned}
$$

\subsection{Assumption 6.4 is satisfied by the universal hermitian} element $\chi$.

Proposition. Let $\partial_{\chi: B} \xi$ be defined for $\xi \in \mathcal{L} \mathcal{R}(\chi ; B)$ by

$$
\partial_{\chi: B} \xi=\frac{i}{2}(1 \otimes(1-u))\left(\partial_{u: b}^{\mathcal{Q}} \xi\right)((1-u) \otimes 1) .
$$

Then $\partial_{\chi: B}$ is a derivation of $\mathcal{L} \mathcal{R}(\chi ; B)$ into $\mathcal{L} \mathcal{R}(\chi ; B) \otimes \mathcal{L} \mathcal{R}(\chi ; B)$ such that

$$
\left(\operatorname{id}_{\mathfrak{M}_{n}} \otimes \partial_{\chi: B}\right) \widetilde{\mathcal{R}}_{n}(\chi ; B)(\nu)=\widetilde{\mathcal{R}}_{n}(\chi ; B)(\nu) \otimes_{\mathfrak{M}_{n}} \widetilde{\mathcal{R}}_{n}(\chi ; B)(\nu)
$$

for all $n \in \mathbb{N}$ and $\nu \in \widetilde{\rho}_{n}(\chi ; B)$.

Proof. Remark that $\partial_{\chi: B}$ is well-defined as a derivation of $\mathcal{L} \mathcal{R}(\chi ; B)$ into $\mathcal{Q R}(u ; B)$ in view of the results in 12.2 and 12.4 and of the fact that the map $\eta \rightsquigarrow-i(1 \otimes(1-u)) \eta((1-u) \otimes 1)$ is a $\mathcal{Q R}(u ; B)$-bimodule map of $\mathcal{Q R}(u ; B) \otimes \mathcal{Q R}(u ; B)$ into itself. It will suffice to prove the formula for $\left(\operatorname{id}_{\mathfrak{M}_{n}} \otimes \partial_{\chi: B}\right) \widetilde{\mathcal{R}}_{n}(\chi ; B)(\nu)$, since this formula completely determines $\partial_{\chi: B}$ and establishes that its range lies in $\mathcal{L} \mathcal{R}(\chi ; B) \otimes \mathcal{L R}(\chi ; B)$.

Using Lemma 12.4 a) and c) we have:

$$
\begin{aligned}
& \widetilde{\mathcal{R}}_{n}(\chi ; B)(\nu)=\frac{i}{2}\left(\widetilde{\mathcal{R}}_{n}(u ; B)(\sigma)\right)\left(I_{n} \otimes(1-u)\right) \\
& +\frac{i}{2}\left(I_{n} \otimes u\right)\left(\widetilde{\mathcal{R}}_{n}(u ; B)(\sigma)\right)\left(I_{n} \otimes(u-1)\right)
\end{aligned}
$$




$$
\begin{aligned}
& +\frac{i}{2}\left(I_{n} \otimes(u-1)\right) \\
& =\frac{i}{2}\left(\left(I_{n} \otimes(1-u)\right)\left(\widetilde{\mathcal{R}}_{n}(u ; B)(\sigma)\right)\left(I_{n} \otimes(1-u)\right)-I_{n} \otimes(1-u)\right) .
\end{aligned}
$$

Since

$$
\begin{aligned}
& \left(\operatorname{id}_{\mathfrak{M}_{n}} \otimes \partial_{\chi: B}\right)\left(\widetilde{\mathcal{R}}_{n}(\chi ; B)(\nu)\right) \\
& \left.=\frac{i}{2}\left(I_{n} \otimes 1 \otimes(1-u)\right)\left(\operatorname{id}_{\mathfrak{M}_{n}} \otimes \partial_{u: B}^{\mathcal{Q}}\right)\left(\widetilde{\mathcal{R}}_{n}(\chi ; B)(\nu)\right)\left(I_{n} \otimes(1-u) \otimes 1\right)\right)
\end{aligned}
$$

and

$$
\begin{aligned}
& \left(\operatorname{id}_{\mathfrak{M}_{n}} \otimes \partial_{u: B}^{\mathcal{Q}}\right)\left(\widetilde{\mathcal{R}}_{n}(\chi ; B)(\nu)\right) \\
& =\frac{i}{2}\left(-\left(I_{n} \otimes 1\right) \otimes_{\mathfrak{M}_{n}}\left(\left(\widetilde{\mathcal{R}}_{n}(u ; B)(\sigma)\right)\left(I_{n} \otimes(1-u)\right)\right)\right. \\
& -\left(\left(I_{n} \otimes(1-u)\right)\left(\widetilde{\mathcal{R}}_{n}(u ; B)(\sigma)\right) \otimes_{\mathfrak{M}_{n}}\left(I_{n} \otimes 1\right)+I_{n} \otimes 1 \otimes 1\right. \\
& +\left(\left(I_{n} \otimes(1-u)\right)\left(\widetilde{\mathcal{R}}_{n}(u ; B)(\sigma)\right) \otimes_{\mathfrak{M}_{n}}\left(\left(\widetilde{\mathcal{R}}_{n}(u ; B)(\sigma)\right)\left(I_{n} \otimes(1-u)\right)\right)\right.
\end{aligned}
$$

we get that

$$
\begin{aligned}
& \left(\operatorname{id}_{\mathfrak{M}_{n}} \otimes \partial_{\chi: B}\right)\left(\widetilde{\mathcal{R}}_{n}(\chi ; B)(\nu)\right) \\
& =\frac{1}{4}\left(\left(I_{n} \otimes(1-u)\right) \otimes_{\mathfrak{M}_{n}}\left(I_{n} \otimes(1-u)\right)\left(\widetilde{\mathcal{R}}_{n}(u ; B)(\sigma)\right)\left(I_{n} \otimes(1-u)\right)\right. \\
& +\left(I_{n} \otimes(1-u)\right)\left(\widetilde{\mathcal{R}}_{n}(u ; B)(\sigma)\right)\left(I_{n} \otimes(1-u)\right) \otimes_{\mathfrak{M}_{n}}\left(I_{n} \otimes(1-u)\right) \\
& -\left(I_{n} \otimes(1-u)\right) \otimes_{\mathfrak{M}_{n}}\left(I_{n} \otimes(1-u)\right) \\
& -\left(I_{n} \otimes(1-u)\right)\left(\widetilde{\mathcal{R}}_{n}(u ; B)(\sigma)\right)\left(I_{n} \otimes(1-u)\right) \\
& \left.\otimes_{\mathfrak{M}_{n}}\left(I_{n} \otimes(1-u)\right)\left(\widetilde{\mathcal{R}}_{n}(u ; B)(\sigma)\right)\left(I_{n} \otimes(1-u)\right)\right) \\
& =-\frac{1}{4}\left(\left(I_{n} \otimes(1-u)\right) \widetilde{\mathcal{R}}_{n}(u ; B)(\sigma)\left(I_{n} \otimes(1-u)\right)-I_{n} \otimes(1-u)\right) \\
& \otimes_{\mathfrak{M}_{n}}\left(\left(I_{n} \otimes(1-u)\right) \widetilde{\mathcal{R}}_{n}(u ; B)(\sigma)\left(I_{n} \otimes(1-u)\right)-I_{n} \otimes(1-u)\right) \\
& =-\frac{1}{4}\left(-2 i \widetilde{\mathcal{R}}_{n}(\chi ; B)(\nu)\right) \otimes_{\mathfrak{M}_{n}}\left(-2 i \widetilde{\mathcal{R}}_{n}(\chi ; B)(\nu)\right) \\
& =\left(\widetilde{\mathcal{R}}_{n}(\chi ; B)(\nu)\right) \otimes_{\mathfrak{M}_{n}}\left(\widetilde{\mathcal{R}}_{n}(\chi ; B)(\nu)\right) .
\end{aligned}
$$

13. The SERIES EXPANSION AT THE ORIGIN OF A FUlLy MATRICIAL ANALYTIC FUNCTION

13.1. Since we will deal with a local question, we will work in the affine framework and we will use only the Banach space structure of $B$ (like in section 7). We will assume throughout sections $13.1-13.8$ that $B$ is 
finite-dimensional and then in section 13.9 give the general nonsense argument extending the result about the series expansion to the case of general $B$.

Let $\Omega=\left(\Omega_{n}\right)_{n \geq 1}$ be a fully matricial open $B$-set so that $\Omega_{n} \ni 0_{n}$ (the zero element of $\mathfrak{M}_{n}(B)$ ) and let $f=\left(f_{n}\right)_{n \geq 1} \in \mathcal{A}(\Omega)$. The series expansion of $f_{n}$ at $0_{n}$ is

$$
f_{n}(\beta)=f_{n}\left(0_{n}\right)+\sum_{k \geq 1} \frac{1}{k !} d^{k} f_{n}\left(0_{n}\right) \underbrace{[\beta, \ldots, \beta]}_{k \text {-times }}
$$

where $d^{k} f_{n}\left(0_{n}\right)$ is viewed as a symmetric $k$-linear map of $\mathfrak{M}_{n}(B) \times$ $\ldots \times \mathfrak{M}_{n}(B)\left(k\right.$-times) into $\mathfrak{M}_{n}$ and where it is assumed that some disk $\{z \beta|z \in \mathbb{C}| z \mid,<1+\varepsilon\}$ is in $\Omega_{n}$. Our aim here is to identify the $\left(d^{k} f_{n}\left(0_{n}\right)\right)_{n \geq 1, k \geq 1}$ which occur taking into account the "fully matricial" - conditions (convergence question aside).

13.2. Let $F_{n, k}(\beta)=d^{k} f_{n}\left(0_{n}\right)[\beta, \ldots, \beta]$, where $\beta \in \mathfrak{M}_{n}(B)$.

Lemma. We have $\left(F_{n, k}\right)_{n \geq 1} \in \mathcal{A}\left(\mathfrak{M}_{n}(B)\right)$.

Proof. The lemma is an immediate consequence of taking $k$-th order differentials at the origin of the two equalities (equivariance and direct sum conditions) which are satisfied by the fully matricial function $f$.

13.3. Since $F_{n, k}$ was derived from $d^{k} f_{n}\left(0_{n}\right)$, there are linear maps $\Phi_{n, k}$ : $\left(\mathfrak{M}_{n}\right)^{\otimes k} \otimes B^{\otimes k} \rightarrow \mathfrak{M}_{n}$, satisfying the symmetry condition

$$
\begin{aligned}
& \Phi_{n, k}\left(A_{1} \otimes \cdots \otimes A_{k} \otimes b_{1} \otimes \cdots \otimes b_{k}\right) \\
& =\Phi_{n, k}\left(A_{\sigma(1)} \otimes \cdots \otimes A_{\sigma(k)} \otimes b_{\sigma(1)} \otimes \cdots \otimes b_{\sigma(k)}\right)
\end{aligned}
$$

for all $\sigma \in \mathfrak{S}_{k}$, so that

$$
F_{n, k}(A \otimes b)=\Phi_{n, k}(A \otimes \cdots \otimes A \otimes b \otimes \cdots \otimes b) .
$$

To recover $\Phi_{n, k}$ from $F_{n, k}$ one uses the fact that $k ! \Phi_{n, k}\left(A_{1} \otimes \cdots \otimes A_{k} \otimes\right.$ $\left.b_{1} \otimes \cdots \otimes b_{k}\right)$ is the coefficient of $\lambda_{1} \ldots \lambda_{k}$ in $F_{n, k}\left(\lambda_{1} A_{1} \otimes b_{1}+\cdots+\right.$ $\left.\lambda_{k} A_{k} \otimes b_{k}\right)$ which is a polynomial of degree $k$ in $\lambda_{1}, \ldots, \lambda_{k} \in \mathbb{C}$. The properties of $\left(F_{n, k}\right)_{n \geq 1}$ in Lemma 13.2, then translate immediately into properties of the $\Phi_{n, k}$ which we record in the next lemma.

Lemma. a) We have

$$
\begin{aligned}
& \Phi_{n, k}\left(S A_{1} S^{-1} \otimes \cdots \otimes S A_{k} S^{-1} \otimes b_{1} \otimes \cdots \otimes b_{k}\right) \\
& =S \Phi_{n, k}\left(A_{1} \otimes \cdots \otimes A_{k} \otimes b_{1} \otimes \cdots \otimes b_{k}\right) S^{-1},
\end{aligned}
$$

where $S \in G L(n, \mathbb{C})$. 
b) If $n=n^{\prime}+n^{\prime \prime}$ and if we put

$$
\begin{gathered}
\widetilde{\Phi}_{n, k}\left(A_{1} \otimes b_{1} \otimes A_{2} \otimes b_{2} \otimes \cdots \otimes A_{k} \otimes b_{k}\right) \\
=\Phi_{n, k}\left(A_{1} \otimes \cdots \otimes A_{k} \otimes b_{1} \otimes \cdots \otimes b_{k}\right),
\end{gathered}
$$

we then have

$$
\begin{aligned}
& \widetilde{\Phi}_{n, k}\left(\left(A_{1}^{\prime} \otimes b_{1}^{\prime}\right) \oplus\left(A_{1}^{\prime \prime} \otimes b_{1}^{\prime \prime}\right)\right) \otimes \cdots \otimes\left(\left(A_{k}^{\prime} \otimes b_{k}^{\prime}\right) \oplus\left(A_{k}^{\prime \prime} \otimes b_{k}^{\prime \prime}\right)\right) \\
& =\widetilde{\Phi}_{n^{\prime}, k}\left(A_{1}^{\prime} \otimes b_{1}^{\prime} \otimes \cdots \otimes A_{k}^{\prime} \otimes b_{k}^{\prime}\right) \oplus \widetilde{\Phi}_{n^{\prime \prime}, k}\left(A_{1}^{\prime \prime} \otimes b_{1}^{\prime \prime} \otimes \cdots \otimes A_{k}^{\prime \prime} \otimes b_{k}^{\prime \prime}\right)
\end{aligned}
$$

where $A_{j}^{\prime} \in \mathfrak{M}_{n^{\prime}}, A_{j}^{\prime \prime} \in \mathfrak{M}_{n^{\prime \prime}}, b_{j}^{\prime}, b_{j}^{\prime \prime} \in B$.

13.4. Let $\rho_{n, k} \in \mathfrak{S}_{k} \rightarrow \mathcal{L}\left(\left(\mathbb{C}^{n}\right)^{\otimes k}\right) \simeq \mathfrak{M}_{n}^{\otimes k}$ be the representation of $\mathfrak{S}_{k}$ which permutes the $\mathbb{C}^{n}$ factors in the $k$-fold tensor product.

Lemma. Let $\Phi_{n, k}:\left(\mathfrak{M}_{n}\right)^{\otimes k} \otimes B^{\otimes k} \rightarrow \mathfrak{M}_{n}$ be linear maps satisfying conditions a) and b) of Lemma 13.3 and let $\Psi_{n, k}:\left(\mathfrak{M}_{n}\right)^{\otimes(k+1)} \otimes B^{\otimes k} \rightarrow$ $\mathbb{C}$ denote the map $\Psi_{n, k}\left(A_{1} \otimes \cdots \otimes A_{k+1} \otimes b_{1} \otimes \cdots \otimes b_{k}\right)=\operatorname{Tr}\left(\Phi_{n, k}\left(A_{1} \otimes\right.\right.$ $\left.\left.\cdots \otimes A_{k} \otimes b_{1} \otimes \cdots \otimes b_{k}\right) A_{k+1}\right)$. Then there are $\varphi_{\sigma} \in\left(B^{\otimes k}\right)^{d}$ so that

$$
\begin{aligned}
& \Psi_{n, k}\left(A_{1} \otimes \cdots \otimes A_{k+1} \otimes b_{1} \otimes \cdots \otimes b_{k}\right) \\
& =\sum_{\sigma \in \mathfrak{S}_{k+1}} \operatorname{Tr}\left(\left(A_{1} \otimes \cdots \otimes A_{k+1}\right) \rho_{n, k+1}(\sigma)\right) \varphi_{\sigma}\left(b_{1} \otimes \cdots \otimes b_{k}\right)
\end{aligned}
$$

for all $n \in \mathbb{N}$. If $n \geq k+1$ the $\varphi_{\sigma}$ for which the previous formula holds for that value of $n$ are unique.

Proof. Condition a) implies that

$$
\begin{aligned}
& \Psi_{n, k}\left(S A_{1} S^{-1} \otimes \cdots \otimes S A_{k+1} S^{-1} \otimes b_{1} \otimes \cdots \otimes b_{k}\right) \\
& =\Psi\left(A_{1} \otimes \cdots \otimes A_{k+1} \otimes b_{1} \otimes \cdots \otimes b_{k}\right) .
\end{aligned}
$$

Keeping $n$ and $b_{1}, \ldots, b_{k}$ fixed, this is a linear map $\operatorname{Tr}(\cdot X)$ for some $X \in\left(\mathfrak{M}_{n}\right)^{k+1}$ and we must have $S^{\otimes(k+1)} X\left(S^{\otimes(k+1)}\right)^{-1}=X$ for $S \in$ $G L(n ; \mathbb{C})$. By the theorem of Weyl $X=\sum_{\sigma \in \mathfrak{S}_{k+1}} c_{\sigma} \rho_{n, k+1}(\sigma)$. It is easy to see that for fixed $n \in \mathbb{N}$ we can find $\varphi_{\sigma} \in\left(B^{\otimes k}\right)^{d}$ so that we will have $X=\sum_{\sigma \in \mathfrak{S}_{k+1}} \varphi_{\sigma}\left(b_{1} \otimes \cdots \otimes b_{k}\right) \rho_{n, k+1}(\sigma)\left(\varphi_{\sigma}\right.$ is the linear map interpolating between the coefficient $c_{\sigma}$ for the $b_{1} \otimes \cdots \otimes b_{k}$ with the $b_{j}$ 's running over a fixed basis of $B$ ).

We still have to prove that the functionals $\varphi_{\sigma}$ can be chosen independent of $n$. This can be seen as follows. On one hand if $n \geq k+1$ the $\rho_{n, k+1}(\sigma), \sigma \in \mathfrak{S}_{k+1}$, are linearly independent and the coefficients $c_{\sigma}$ and functionals $\varphi_{\sigma}$ are unique. On the other hand condition b) in Lemma 13.3 applied to $b_{j}^{\prime}=b_{j}^{\prime \prime}$ and $A_{j}^{\prime \prime}=0$ shows that

$$
\Phi_{n, k}\left(\left(A_{1}^{\prime} \oplus 0\right) \otimes \cdots \otimes\left(A_{k}^{\prime} \oplus 0\right) \otimes b_{1} \otimes \cdots \otimes b_{k}\right)
$$




$$
=\Phi_{n^{\prime}, k}\left(A_{1}^{\prime} \otimes \cdots \otimes A_{k}^{\prime} \otimes b_{1} \otimes \cdots \otimes b_{k}\right) \oplus 0_{n^{\prime \prime}}
$$

which implies

$$
\begin{aligned}
& \Psi_{n^{\prime}, k}\left(A_{1}^{\prime} \otimes \cdots \otimes A_{k+1}^{\prime} \otimes b_{1} \otimes \cdots \otimes b_{k}\right) \\
& =\Psi_{n, k}\left(\left(A_{1}^{\prime} \oplus 0\right) \otimes \cdots \otimes\left(A_{k+1}^{\prime} \oplus 0\right) \otimes b_{1} \otimes \cdots \otimes b_{k}\right) .
\end{aligned}
$$

Since

$$
\begin{aligned}
& \operatorname{Tr}\left(\left(A_{1}^{\prime} \otimes \cdots \otimes A_{k+1}^{\prime}\right) \rho_{n^{\prime}, k+1}(\sigma)\right) \\
& =\operatorname{Tr}\left(\left(\left(A_{1}^{\prime} \oplus 0\right) \otimes \cdots \otimes\left(A_{k+1}^{\prime} \oplus 0\right)\right) \rho_{n, k+1}(\sigma)\right),
\end{aligned}
$$

we infer that the $\varphi_{\sigma}$ which work for $n$ can also be used for $n^{\prime}<n$. Thus the $\varphi_{\sigma}$ in the formula for some $n \geq k+1$ can be used for all $n \in \mathbb{N}$.

13.5. The result of the preceding section did not draw all the consequences from condition b) of Lemma 13.3 as we shall see in this section.

Remark first that

$$
\operatorname{Tr}\left(\left(A_{1} \otimes \cdots \otimes A_{k+1}\right) \rho_{n, k+1}(\sigma)\right)=\prod_{\left\{\left(i_{1}, \ldots, i_{p}\right) \mid\left(i_{1}, \ldots, i_{p}\right)\right.} \operatorname{Tr}\left(A_{i_{p}} \ldots A_{i_{1}}\right),
$$

(the product is over disjoint cycles of $\sigma$, that is without repetitions). Let $C_{k+1} \subset \mathfrak{S}_{k+1}$ be the cycles of length $k+1$.

Lemma. The conclusion of Lemma 13.4 holds with the sum taken only over $C_{k+1}$ (that is $\varphi_{\sigma}=0$ if $\sigma \notin C_{k+1}$ ).

Proof. We begin by remarking that Lemma 13.3 b) implies

$$
\begin{aligned}
& \Psi_{n m, k}(\underbrace{\left(A_{1} \oplus \cdots \oplus A_{1}\right)}_{m} \otimes \cdots \otimes \underbrace{\left(A_{k+1} \oplus \cdots \oplus A_{k+1}\right)}_{m} \otimes b_{1} \otimes \cdots \otimes b_{k}) \\
& =\operatorname{Tr}\left(\widetilde{\Phi}_{n m, k}\left(\left(A_{1} \oplus \cdots \oplus A_{1}\right) \otimes b_{1} \otimes \cdots\left(A_{k} \oplus \cdots \oplus A_{k}\right) \otimes b_{k} \otimes\left(A_{k+1} \oplus \cdots \oplus A_{k+1}\right)\right)\right. \\
& =m \operatorname{Tr}\left(\Phi_{n, k}\left(A_{1} \otimes \cdots \otimes A_{k} \otimes b_{1} \otimes \cdots \otimes b_{k}\right) A_{k+1}\right) \\
& =m \Psi_{n, k}\left(A_{1} \otimes \cdots \otimes A_{k+1} \otimes b_{1} \otimes \cdots \otimes b_{k}\right) .
\end{aligned}
$$

On the other hand, if $c(\sigma)$ is the number of cycles of the permutation $\sigma$, we have

$$
\begin{aligned}
& \operatorname{Tr}\left(\left(\left(A_{1} \oplus \cdots \oplus A_{1}\right) \otimes \cdots \otimes\left(A_{k+1} \oplus \cdots \oplus A_{k+1}\right)\right) \rho_{m n}(\sigma)\right) \\
& =\prod_{\left(i_{1} \ldots i_{p}\right)} \operatorname{Trcycle~of~} \sigma \\
& =\prod_{\left(i_{1}, \ldots, i_{p}\right) \text { cycle of } \sigma} m \operatorname{Tr}\left(\left(A_{i_{p}} \oplus \cdots \oplus A_{i_{p}}\right) \ldots\left(A_{i_{1}} \oplus \cdots \oplus A_{i_{1}}\right)\right) \\
& =m^{c(\sigma)} \operatorname{Tr}\left(\left(A_{1} \otimes \cdots \otimes A_{i_{1}}\right)\right. \\
& \left.\left.=A_{k+1}\right) \rho_{n}(\sigma)\right) .
\end{aligned}
$$


Hence Lemma 13.4 applied to $n m$ and $\underbrace{A_{j} \oplus \cdots \oplus A_{j}}_{m}$ instead of $n$ and $A_{j}$ gives that

$$
\begin{aligned}
& m \Psi_{n, k}\left(A_{1} \otimes \cdots \otimes A_{k+1} \otimes b_{1} \otimes \cdots \otimes b_{k}\right) \\
& =\sum_{\sigma \in \mathfrak{S}_{k+1}} m^{c(\sigma)} \operatorname{Tr}\left(\left(A_{1} \otimes \cdots \otimes A_{k+1}\right) \rho_{n, k+1}(\sigma)\right) \varphi_{\sigma}\left(b_{1} \otimes \cdots \otimes b_{k}\right) .
\end{aligned}
$$

By the uniqueness of the $\varphi_{\sigma}$ when $n \geq k+1$ we infer that $\varphi_{\sigma}=0$ if $c(\sigma)>1$.

13.6. We shall now translate the result of 13.5 in terms of the $\Phi_{n, k}$. Since $C_{k+1}$ is parametrized by $\mathfrak{S}_{k}$ by putting each cycle in the form $\pi=(k+1, \theta(k), \ldots, \theta(1))$ for some $\theta \in \mathfrak{S}_{k}$, we have that

$$
\operatorname{Tr}\left(\left(A_{1} \otimes \cdots \otimes A_{k+1}\right) \rho_{n, k+1}(\pi)\right)=\operatorname{Tr}\left(A_{\theta(1)} \ldots A_{\theta(k)} A_{k+1}\right) .
$$

Lemma. Let $\Phi_{n, k}:\left(\mathfrak{M}_{n}\right)^{\otimes k} \otimes B^{\otimes k} \rightarrow \mathfrak{M}_{n}$ be linear maps satisfying conditions a) and b) of Lemma 13.3. Then there are $\psi_{\theta} \in\left(B^{\otimes k}\right)^{d}$ for $\theta \in \mathfrak{S}_{k}$ so that

$\Phi_{n, k}\left(A_{1} \otimes \cdots \otimes A_{k} \otimes b_{1} \otimes \cdots \otimes b_{k}\right)=\sum_{\theta \in \mathfrak{S}_{k}} \psi_{\theta}\left(b_{1} \otimes \cdots \otimes b_{k}\right) A_{\theta(1)} \ldots A_{\theta(k)}$

for all $n \in \mathbb{N}$. If $n \geq k+1$ the $\psi_{\theta}$ are unique.

Proof. In view of the remarks preceding the Lemma, this is an immediate consequence of Lemma 13.5 with $\psi_{\theta}=\varphi_{\pi}$, where $\pi=$ $(k+1, \theta(k), \ldots, \theta(1))$.

13.7. Since $B$ is finite-dimensional, we can express $\widetilde{\Phi}_{n, k}$ in terms of a basis $\varphi_{1}, \ldots, \varphi_{N}$ of $B^{d}$. We will use for this the fully matricial functions $z(\varphi)$ defined in 7.1 .

Lemma. Let $\Phi_{n, k}:\left(\mathfrak{M}_{n}\right)^{\otimes k} \otimes B^{\otimes k} \rightarrow \mathfrak{M}_{n}$ be linear maps satisfying conditions a) and b) of Lemma 13.3 and let $\widetilde{\Phi}_{n, k}$ be defined as in Lemma 13.3. Then there are numbers $a(\theta ; j(1), \ldots, j(k))$, where $\theta \in \mathfrak{S}_{k}$ and $1 \leq j(1), \ldots, j(k) \leq N$, so that

$$
\begin{aligned}
& \widetilde{\Phi}_{n, k}\left(A_{1} \otimes b_{1} \otimes \cdots \otimes A_{k} \otimes b_{k}\right) \\
& =\sum_{\theta \in \mathfrak{S}_{k}} \sum_{\substack{1 \leq j(p) \leq N \\
1 \leq p \leq k}} a(\theta ; j(1), \ldots, j(k)) z\left(\varphi_{j(1)}\right)_{n}\left(A_{\theta(1)} \otimes b_{\theta(1)}\right) \ldots z\left(\varphi_{j(k)}\right)\left(A_{\theta(k)} \otimes b_{\theta(k)}\right)
\end{aligned}
$$

for all $n \in \mathbb{N}$. If $n \geq k+1$, the numbers $a(\theta ; j(1), \ldots, j(k))$ are unique. 
Proof. This is essentially a reformulation of Lemma 13.6. We define the numbers $a(\theta ; j(1), \ldots, j(k))$ so that

$\psi_{\theta}\left(b_{1} \otimes \cdots \otimes b_{k}\right)=\sum_{\substack{1 \leq j(p) \leq N \\ 1 \leq p \leq k}} a(\theta ; j(1), \ldots, j(k)) \varphi_{j(1)}\left(b_{\theta(1)}\right) \ldots \varphi_{j(k)}\left(b_{\theta(k)}\right)$.

Remark that given $\psi_{\theta}$, these numbers are unique, which will give uniqueness of the numbers $a(\ldots)$ once the $\psi_{\theta}$ are unique for $n \geq k+1$. The fact that this lemma is a consequence of 13.6 becomes obvious after noting that

$$
z(\varphi)_{n}(A \otimes b)=\varphi(b) A
$$

Corollary. If in the preceding lemma the $\widetilde{\Phi}_{n, k}$ satisfy the symmetry condition

$\widetilde{\Phi}_{n, k}\left(A_{\alpha(1)} \otimes b_{\alpha(1)} \otimes \cdots \otimes A_{\alpha(k)} \otimes b_{\alpha(k)}\right)=\widetilde{\Phi}_{n, k}\left(A_{1} \otimes b_{1} \otimes \cdots \otimes A_{k} \otimes b_{k}\right)$

for all $\alpha \in \mathfrak{S}_{k}$, then the numbers $a(\theta ; j(1), \ldots, j(k))$ will not depend on $\theta$.

Proof. This follows immediately from the uniqueness part of the lemma.

13.8. We are now ready to return to the context of 13.1 and to describe the series expansion of $f \in \mathcal{A}(\Omega)$ at the origin. We will assume $\varphi_{1}, \ldots, \varphi_{N}$ is a basis of $B^{d}$.

Theorem. If $\Omega$ is a fully matricial affine $B$-set containing the origin and $f \in \mathcal{A}(\Omega)$, then for each $k \in \mathbb{N}$ there are numbers $a_{k}(j(1), \ldots, j(k))$ so that

$$
\begin{aligned}
& d^{k} f_{n}\left(0_{n}\right)\left[\beta_{1}, \ldots, \beta_{k}\right] \\
& =\sum_{\theta \in \mathfrak{S}_{k}} \sum_{\substack{1 \leq j(p) \leq N \\
1 \leq p \leq k}} a_{k}(j(1), \ldots, j(k)) z\left(\varphi_{j(1)}\right)_{n}\left(\beta_{\theta(1)}\right) \ldots z\left(\varphi_{j(k)}\right)\left(\beta_{\theta(k)}\right) .
\end{aligned}
$$

Equivalently if $F_{k}=\left(F_{n, k}\right)_{n \geq 1}$ where

$$
F_{n, k}(\beta)=d^{k} f_{n}\left(0_{n}\right)[\beta, \ldots, \beta]
$$

then

$$
(k !)^{-1} F_{k}=\sum_{\substack{1 \leq j(p) \leq N \\ 1 \leq p \leq k}} a_{k}(j(1), \ldots, j(k)) z\left(\varphi_{j(1)}\right) \ldots z\left(\varphi_{j(k)}\right) .
$$


The formula for $d^{k} f_{n}\left(0_{n}\right)$ determines the numbers $a_{k}$ uniquely if $n \geq$ $k+1$. The series expansion of $f$ at the origin is

$$
f=\left(f_{n}\left(0_{n}\right)\right)_{n \geq 1}+\sum_{k \geq 1} \sum_{\substack{1 \leq j(p) \leq N \\ 1 \leq p \leq k}} a_{k}(j(1), \ldots, j(k)) z\left(\varphi_{j(1)}\right) \ldots z\left(\varphi_{j(k)}\right) .
$$

Proof. This follows easily from an application of Lemma 13.7 and Corollary 13.7 to

$$
\Phi_{n, k}\left(A_{1} \otimes \cdots \otimes A_{k} \otimes b_{1} \otimes \cdots \otimes b_{k}\right)=d^{k} f_{n}\left(0_{n}\right)\left[A_{1} \otimes b_{1}, \ldots, A_{n} \otimes b_{n}\right]
$$

(that these $\Phi_{n, k}$ have the required properties is a consequence of 13.2 and 13.3).

Remark. The constant term in the series expansions $\left(f_{n}\left(0_{n}\right)\right)_{n \geq 1}$ has a particularly simple form since $f$ is fully maticial $f_{n}\left(0_{n}\right)=f_{1}(0) I_{n} \otimes 1$ or with the notations in 7.1 we can write $f_{1}(0) \mathbb{1}$ for $\left(f_{n}\left(0_{n}\right)\right)_{n \geq 1}$.

13.9. The series expansion for $\mathcal{X}$-valued fully matricial analytic functions and general $B$. After having dealt with the algebraic questions assuming that $\operatorname{dim} B<\infty$ and that the fully matricial analytic function $f$ is scalar-valued, it is now easy to remove the restriction on the dimension and get the result for an arbitrary Banach space $B$ and additionally allow the function $f$ to be $\mathcal{X}$-valued, where $\mathcal{X}$ is some other complex Banach space.

If $\alpha \in \mathcal{L}\left(B^{\widehat{\otimes} k}, \mathcal{X}\right)$ where $\widehat{\otimes} k$ is the $k$-th projective tensor power of $B$, then $\alpha$ is a bounded $k$-linear map of $B \times \ldots \times B$ ( $k$-times) into $\mathcal{X}$. We define a fully matricial analytic function $\mathcal{Z}_{k}(\alpha)$ on $\mathfrak{M}(B)$ with values in $\mathcal{X}$ by

$$
\mathcal{Z}_{k}(\alpha)_{n}(\beta)=\left(\operatorname{id}_{\mathfrak{M}_{n}} \otimes \alpha\right)\left(\beta \otimes_{\mathfrak{M}_{n}} \cdots \otimes_{\mathfrak{M}_{n}} \beta\right)
$$

where $\beta \in \mathfrak{M}_{n}(B)$ and the last $\otimes_{\mathfrak{M}_{n}}$ is $k$-fold. If $\beta=\sum_{1 \leq i, j \leq n} e_{i j} \otimes b_{i j}$ this means

$$
\begin{aligned}
\mathcal{Z}_{k}(\alpha)_{n}(\beta) & =\sum_{1 \leq i_{1}, \ldots, i_{k+1} \leq n} e_{i_{1} i_{2}} \ldots e_{i_{k} i_{k+1}} \otimes \alpha\left(b_{i_{1} i_{2}} \otimes \ldots b_{i_{k} i_{k+1}}\right) \\
& =\sum_{1 \leq i_{1}, \ldots, i_{k+1} \leq n} e_{i_{1} i_{k+1}} \otimes \alpha\left(b_{i_{1} i_{2}} \otimes b_{i_{2} i_{3}} \otimes \cdots \otimes b_{i_{k} i_{k+1}}\right) .
\end{aligned}
$$

It is easily seen that $\mathcal{Z}_{k}(\alpha)=\left(\mathcal{Z}_{k}(\alpha)_{n}\right)_{n \geq 1}$ is a fully matricial analytic function on $\mathfrak{M}(B)$ with values in $\mathcal{X}$.

If $k=1$ and $\mathcal{X}=\mathbb{C}$ then $z(\varphi)$ defined in 7.1 coincides with $\mathcal{Z}_{1}(\varphi)$. Also if $\varphi_{1}, \ldots, \varphi_{k} \in B^{d}$ then $\varphi_{1} \otimes \cdots \otimes \varphi_{k} \in \mathcal{L}\left(B^{\otimes k}, \mathbb{C}\right)$ and $z\left(\varphi_{1}\right) \ldots z\left(\varphi_{k}\right)=$ $\mathcal{Z}_{k}\left(\varphi_{1} \otimes \cdots \otimes \varphi_{k}\right)$. If $\operatorname{dim} B<\infty$ then Theorem 13.8 is precisely the fact that $\left(d^{k} f(0)\right)_{n}[\beta, \ldots, \beta]$ equals $\left(\mathcal{Z}_{k}(\alpha)\right)_{n}(\beta)$ for some $\alpha \in \mathcal{L}\left(B^{\otimes k}, \mathbb{C}\right)$. 
Note also that taking $n=k+1$ and $\beta=e_{12} \otimes b_{1}+\cdots+e_{k k+1} \otimes b_{k}$ we have

$$
\mathcal{Z}_{k}(\alpha)_{k+1}(\beta)=e_{1 k+1} \otimes \alpha\left(b_{1} \otimes \cdots \otimes b_{k}\right)
$$

Theorem. If $\Omega$ is a fully matricial affine B-set containing the origin and $f$ is a fully matricial $\mathcal{X}$-valued analytic function on $\Omega$, then for each $k \in \mathbb{N}$ there is a unique $\alpha_{k} \in \mathcal{L}\left(B^{\widehat{\otimes} k}, \mathcal{X}\right)$ so that

$$
d^{k} f_{n}\left(0_{n}\right)[\beta, \ldots, \beta]=\mathcal{Z}_{k}\left(\alpha_{k}\right)_{n}(\beta)
$$

for $\beta \in \mathfrak{M}_{n}(B)$. The series expansion of $f$ at the origin is

$$
f_{1}(0) \mathbb{1}+\sum_{k \geq 1}(k !)^{-1} \mathcal{Z}_{k}\left(\alpha_{k}\right)
$$

Proof. The case $\operatorname{dim} B<\infty$ and $\mathcal{X}=\mathbb{C}$ is just Theorem 13.8 as already noted. Also if $\operatorname{dim} B<\infty$ and $N=\operatorname{dim} \mathcal{X}<\infty$ we have that $f={ }_{1} f \otimes x_{1}+\cdots+{ }_{N} f \otimes x_{N}$ where ${ }_{j} f \in A(\Omega), 1 \leq j \leq N$ and $x_{1}, \ldots, x_{N} \in \mathcal{X}$. The theorem in this case follows from the theorem applied to each of the ${ }_{j} f$.

Next, assume $\operatorname{dim} B<\infty$, but place no restriction on $\mathcal{X}$. For each $\varphi \in \mathcal{X}^{d}$ there is $\alpha_{\varphi} \in \mathcal{L}\left(B^{\otimes k}, \mathbb{C}\right)$ so that

$$
d^{k}(\mathfrak{M}(\varphi) \circ f)(0)=\mathcal{Z}_{k}\left(\alpha_{\varphi}\right) .
$$

Both sides being linear in $\varphi$, since $\alpha_{\varphi}$ is unique, we infer $\varphi \rightsquigarrow \alpha_{\varphi}$ is a linear map $\mathcal{X}^{d} \rightarrow \mathcal{L}\left(B^{\otimes k}, \mathbb{C}\right)$. It follows that there can be at most $(\operatorname{dim} B)^{k}$ linearly independent $\alpha_{\varphi}$ 's and this implies that the linear span in $\mathcal{X}$ of the union of ranges of $\left(\theta \otimes \operatorname{id}_{\mathcal{X}}\right)\left(\left(d^{k} f\right)(0)\right)_{n}$ with $n \in \mathbb{N}$ is a finite-dimensional subspace $\mathcal{X}_{0} \subset \mathcal{X}$. Thus $\left(d^{k} f\right)(0)$ is essentially a fully-matricial $\mathcal{X}_{0}$-valued analytic function on $\mathfrak{M}(B)$, which is equal to its $k$-th differential at the origin. Hence $\left(d^{k} f\right)(0)=\mathcal{Z}_{k}(\alpha)$ where $\alpha \in$ $\mathcal{L}\left(B^{\otimes k}, \mathcal{X}_{0}\right)$ is viewed as $\mathcal{X}$-valued by composition with the inclusion $\mathcal{X}_{0} \subset \mathcal{X}$

Finally we pass to the general case, i.e., no restrictions on the dimensions of $B$ and $\mathcal{X}$. For each finite dimensional subspace $C$ of $B$ let $f_{C}=f \mid \mathfrak{M}(C)$ and let $\alpha_{C} \in \mathcal{L}\left(C^{\otimes k}, \mathbb{C}\right)$ be such that $d^{k} f_{C}(0)=$ $\mathcal{Z}_{k}\left(\alpha_{C}\right)$. If $C_{1} \subset C_{2}$ we have $\alpha_{C_{2}} \mid C_{1}^{\otimes k}=\alpha_{C_{1}}$. Putting these together yields a linear map $\alpha^{\prime}: B^{\otimes k} \rightarrow \mathbb{C}$. Clearly to conclude the proof it will suffice to show that $\alpha^{\prime}$ extends by continuity to $B^{\widehat{\otimes} k}$ or equivalently that $\alpha^{\prime}$ corresponds to a bounded $k$-linear map $B \times \ldots \times B \rightarrow \mathcal{X}$. If $b_{1}, \ldots, b_{k} \in B$ let $\beta=e_{12} \otimes b_{1}+e_{23} \otimes b_{2}+\cdots+e_{k k+1} \otimes b_{k}$ and recall the remark preceding the statement of the theorem, that $\left(\mathcal{Z}_{k}\left(\alpha_{C}\right)\right)_{k+1}(\beta)=$ 
$e_{1 k+1} \otimes \alpha_{C}\left(b_{1} \otimes \cdots \otimes b_{k}\right)$ where $C=\mathbb{C} b_{1}+\cdots+\mathbb{C} b_{k} \subset B$. It follows that

$$
\left(d^{k} f_{k+1}(0)\right)(\beta)=\left(d^{k} f(0)\right)_{k+1}(\beta)=e_{1 k+1} \otimes \alpha^{\prime}\left(b_{1} \otimes \cdots \otimes b_{k}\right) .
$$

For any crossnorms on $\mathfrak{M}_{n}(B)=\mathfrak{M}_{n} \otimes B$ and $\mathfrak{M}_{n}(\mathcal{X})=\mathfrak{M}_{n} \otimes \mathcal{X}$ there is a constant $K$ such that

$$
\left\|\left(d^{k} f_{k+1}(0)\right)(\beta)\right\| \leq K\|\beta\|^{k}
$$

which gives for some other constant $K^{\prime}$ that

$$
\left\|\alpha^{\prime}\left(b_{1} \otimes \cdots \otimes b_{k}\right)\right\| \leq K^{\prime}\left(\left\|b_{1}\right\|+\cdots+\left\|b_{k}\right\|\right)^{k} .
$$

If $\left\|b_{1}\right\|=\cdots=\left\|b_{k}\right\|$ we get

$$
\left\|\alpha^{\prime}\left(b_{1} \otimes \cdots \otimes b_{k}\right)\right\| \leq K^{\prime} k^{k}\left\|b_{1}\right\| \ldots\left\|b_{k}\right\| .
$$

Obviously this implies the inequality also without the assumption on the equality of norms.

13.10. The series expansion of a composition of fully matricial analytic functions. This section records the fact that the noncommutative series expansion of a composition of fully matricial holomorphic maps coincides with the composition of the series. More precisely we have the following proposition.

Proposition. Let $B(j)(j=1,2,3)$ be Banach spaces and let $\Omega(j)$ be affine fully matricial $B(j)$-sets containing the origin. Let further $g: \Omega(1) \rightarrow \Omega(2)$ and $f: \Omega(2) \rightarrow \Omega(3)$ be fully matricial holomorphic maps which send the origin to the origin. With the notations of 13.9 let

$$
\begin{aligned}
& \alpha_{k} \in \mathcal{L}\left(B(1)^{\widehat{\otimes} k}, B(2)\right), \\
& \beta_{k} \in \mathcal{L}\left(B(2)^{\widehat{\otimes} k}, B(3)\right), \\
& \gamma_{k}: \mathcal{L}\left(B(1)^{\widehat{\otimes} k}, B(3)\right)
\end{aligned}
$$

be multilinear maps so that

$$
\begin{aligned}
\left(d^{k} g_{n}\right)\left(O_{n}\right)[\beta, \ldots, \beta] & =k ! \mathcal{Z}\left(\alpha_{k}\right)_{n}(\beta) \\
\left(d^{k} f_{n}\right)\left(O_{n}\right)[\beta, \ldots, \beta] & =k ! \mathcal{Z}\left(\beta_{k}\right)_{n}(\beta) \\
\left(d^{k}\left(f_{n} \circ g_{n}\right)\right)\left(O_{n}\right)[\beta, \ldots, \beta] & =k ! \mathcal{Z}\left(\gamma_{k}\right)_{n}(\beta) .
\end{aligned}
$$

Then we have

$$
\gamma_{k}=\sum_{\substack{i_{1}+\cdots+i_{l}=k \\ i_{j} \geq 1}} \beta_{l} \circ\left(\alpha_{i_{1}} \otimes \cdots \otimes \alpha_{i_{l}}\right) .
$$


The proposition follows in a straightforward way from the formulae for $d^{k}(f \circ g)_{n}$ in terms of the differentials of $f_{n}$ and $g_{n}$. Indeed we have

$$
(k !)^{-1} d^{k}(f \circ g)_{n}=\sum_{\substack{1 \leq l \leq k \\ i_{1}+\cdots+i_{l}=k \\ i_{j} \geq 1}}\left(l ! i_{1} ! \ldots i_{l} !\right)^{-1} d^{l} f_{n} \circ\left(d^{i_{1}} g_{n} \otimes \cdots \otimes d^{i_{l}} g_{n}\right)
$$

when applied to $n$ copies of

$$
\beta=\sum_{1 \leq i, j \leq n} e_{i j} \otimes b_{i j}
$$

the left-hand side gives

$$
\sum_{1 \leq p_{1}, \ldots, p_{k+1} \leq n} e_{p_{1} p_{k+1}} \otimes \gamma_{k}\left(b_{p_{1} p_{2}} \otimes \cdots \otimes b_{p_{k} p_{k+1}}\right)
$$

while the right-hand side is

$$
\sum_{1 \leq p_{1}, \ldots, p_{k} \leq n} e_{p_{1} p_{k+1}} \otimes S_{p_{1} \ldots p_{k+1}}
$$

where

$$
\begin{aligned}
S_{p_{1} \ldots p_{k+1}}= & \sum_{\substack{1 \leq l \leq k \\
i_{1}+\cdots+i_{l}=k \\
i_{j} \geq 1}} \beta_{l}\left(\alpha_{i_{1}}\left(b_{p_{1} p_{2}} \otimes \cdots \otimes b_{p_{i_{1}} p_{i_{1}+1}}\right) \cdots\right. \\
& \left.\otimes \alpha_{i_{l}}\left(b_{p_{i_{1}+\cdots+i_{l-1}+1}, p_{i_{1}+\cdots+i_{l-1}+2}} \otimes \cdots \otimes b_{p_{i_{1}+\cdots+i_{l}}, p_{i_{1}+\cdots+i_{l}+1}}\right)\right) .
\end{aligned}
$$

Comparing the two proves the proposition.

\section{The Asymptotic Integral Formula for the Coefficients in the Unit Disk When $B=\mathfrak{M}_{k}$}

14.1. We will build on the series expansion results to study stably matricial analytic functions in the unit disk. We will prove stably matricial analogues of the classical facts about density of polynomials and about the identification of the coefficients with the Fourier coefficients of the restriction to the unit circle. Here the role of the unit circle will be taken by the stably matricial unitary group $\mathcal{U}(B)$. Integration on $\mathcal{U}(B)$ will be the large $N$ limit of integrations with respect to Haar measures. We will assume that $B=\mathfrak{M}_{k}(\mathbb{C})$ in 14.3 and 14.4. The case $B=\mathbb{C}^{k}$ will be the subject of section 16 . 


\subsection{Polynomial approximation for totally bounded analytic functions.}

Definition. A $B_{2}$-valued stably matricial function $f$ on the stably matricial $B_{1}$-set $\Xi$, where $B_{k}$ are $C^{*}$-algebras is totally bounded if for some $t>0$, tf factors through the inclusion $\mathcal{D}_{0}^{c l}\left(B_{2}\right) \subset \mathfrak{M}\left(B_{2}\right)$. We define the uniform norm $\|f\|_{\infty, \Xi}$ of $f$ to be the infimum of the $t^{-1}$ or equivalently to be

$$
\sup _{n \in \mathbb{N}} \sup _{\beta \in \Xi_{n}}\left\|f_{n}(\beta)\right\|
$$

where $\mathfrak{M}_{n}\left(B_{2}\right)$ is endowed with the $C^{*}$-norm. If $\Xi=R \mathcal{D}_{0}\left(B_{1}\right)$ we shall also write $\|f\|_{\infty, R}$ for $\|f\|_{\infty, \Xi}$.

If $\Xi$ is an open stably matricial set, we shall put

$$
\mathbb{H}^{\infty}(\Xi)=\left\{f \in \mathcal{A}(\Xi) \mid\|f\|_{\infty, \Xi}<\infty\right\}
$$

Proposition. If $B$ is a finite-dimensional $C^{*}$-algebra and $f \in \mathbb{H}^{\infty}\left(R^{\prime} \mathcal{D}_{0}(B)\right)$ then given $0<R<R^{\prime}$ and $\varepsilon>0$, there is $Z \in \mathcal{Z}\left(B^{d}\right)$ so that

$$
\|f-Z\|_{\infty, R}<\varepsilon \text {. }
$$

Proof. If $g:\left\{z \in \mathbb{C}|| z \mid<R^{\prime}\right\} \rightarrow \mathcal{X}$, where $\mathcal{X}$ is a Banach space, is an analytic function and if $\sup _{|z|<R^{\prime}}\|g(z)\|=M$ then we have the well-known bound

$$
\sup _{|z| \leq R}\left\|g(z)-\sum_{0 \leq n<N} c_{n} z^{n}\right\| \leq M\left(R / R^{\prime}\right)^{N}\left(1-R / R^{\prime}\right)^{-1}
$$

where $\sum_{n \geq 0} c_{n} z^{n}$ is the series expansion of $g$ at zero.

Since the series expansion at the origin of $f_{n}$ restricted to any onedimensional subspace of $\mathfrak{M}_{n}(B)$ is the series expansion of the restriction of $f_{n}$ to that subspace, we infer that if $\beta \in \mathfrak{M}_{n}(B),\|\beta\| \leq R$ then

$$
\begin{aligned}
& \left\|f_{n}(\beta)-f_{1}(0) I_{n}-\sum_{1 \leq k<N}(k !)^{-1} d^{k} f_{n}\left(0_{n}\right)[\beta, \ldots, \beta]\right\| \\
& \leq C\left(R / R^{\prime}\right)^{N}\left(1-R / R^{\prime}\right)^{-1}
\end{aligned}
$$

where $C$ is the uniform norm of $f$ on $R^{\prime} \mathcal{D}_{0}(B)$.

By Theorem 13.8 and Remark 13.8 this means that

$$
\|f-Z\|_{\infty, R} \leq C\left(R / R^{\prime}\right)^{N}\left(1-R / R^{\prime}\right)^{-1}
$$

for a polynomial function $Z \in \mathbb{Z}\left(B^{d}\right)$. Choosing $N$ large enough the right-hand side will be $<\varepsilon$. 
14.3. The asymptotic integral formula for the coefficients. We precede the proof of the integral formula by some free probability preparations.

Lemma. Let $(A, \varphi)$ be a noncommutative probability space and let $U \in A$ be an invertible element and let $1 \in M \subset A$ be a subalgebra of $A$, so that $M$ and $\left\{U, U^{-1}\right\}$ are free. Assume further that $\varphi\left(U^{k}\right)=\delta_{k, 0}$ for $k \in \mathbb{Z}$ and that $a_{1}, \ldots, a_{m}, b_{1}, \ldots, b_{n}, c \in M$. Then we have

$$
\begin{aligned}
& \varphi\left(a_{1} U a_{2} U \ldots a_{m} U c U^{-1} b_{n} \ldots U^{-1} b_{2} U^{-1} b_{1}\right) \\
& = \begin{cases}0 & \text { if } m \neq n \\
\varphi\left(a_{1} b_{1}\right) \ldots \varphi\left(a_{m} b_{m}\right) \varphi(c) & \text { if } m=n .\end{cases}
\end{aligned}
$$

Proof. We shall denote by $\Phi$ the left-hand side of the equality to be proved. Replacing $U$ by $e^{t} U$ does not change the assumptions of the lemma, but $\Phi$ changes to $e^{t(m-n)} \Phi$. It follows that $\Phi=0$ if $m \neq n$.

Next, observe that it suffices to show that $\varphi(c)=0 \Rightarrow \Phi=0$. Indeed, assuming we have proved this, the formula for $\Phi$ follows by induction over $m=n$. Indeed, if $c-\varphi(c) 1=c^{\prime}$ we have $\varphi\left(c^{\prime}\right)=0$ and

$$
\begin{aligned}
& \varphi\left(a_{1} U \ldots a_{n} U c U^{-1} b_{n} \ldots U^{-1} b_{1}\right) \\
& =\varphi(c) \varphi\left(a_{1} U \ldots a_{n-1} U a_{n} b_{n} U^{-1} b_{n-1} \ldots U^{-1} b_{1}\right)+0
\end{aligned}
$$

and this equals

$$
\varphi(c) \varphi\left(a_{n} b_{n}\right) \varphi\left(a_{1} b_{1}\right) \ldots \varphi\left(a_{n-1} b_{n-1}\right)
$$

if we have proved the formula up to $n-1$.

To conclude the proof, assume that $\varphi(c)=0$ and replace each $a_{j}$ by $\varphi\left(a_{j}\right) 1+a_{j}^{\prime}$ with $\varphi\left(a_{j}^{\prime}\right)=0$ and similarly $b_{j}=\varphi\left(b_{j}\right) 1+b_{j}^{\prime}$ with $\varphi\left(b_{j}^{\prime}\right)=0$. This reduces the proof to showing that

$$
\varphi\left(a_{1} U \ldots a_{n} U c U^{-1} b_{n} \ldots U^{-1} b_{1}\right)=0
$$

when $\varphi(c)=0$ and $a_{j}, b_{j} \in\{1\} \cup(M \cap \operatorname{ker} \varphi)$. In this case

$$
\varphi\left(a_{1} U \ldots a_{n} U c U^{-1} b_{n} \ldots U^{-1} b_{1}\right)
$$

is the expectation of an alternating product of elements from the two sets $M \cap \operatorname{ker} \varphi$ and $\left\{U^{k} \mid k \in \mathbb{Z} \backslash\{0\}\right\}$ and this by freeness equals zero.

Another ingredient in the proof of the integral formula will be an asymptotic freeness result for random Haar unitary matrices which can be found in [18], [14] or [3]. We record it here as the next proposition. 
Proposition. Let $U_{N}$ be the tautological function on $U(N k) \simeq \mathcal{U}\left(N ; \mathfrak{M}_{k}\right)$ with values in $\mathfrak{M}_{N k}$ viewed as an element of the noncommutative probability space $\left(A_{N}, \Phi_{N}\right)$, where $A_{N}=\mathfrak{M}_{N k}\left(L^{\infty}\left(U(N k) ; d \mu_{N}\right)\right)$ and where

$$
\Phi_{N}(T)=\int_{U(N k)}(N k)^{-1} \operatorname{Tr} T d \mu_{N}
$$

with $\mu_{N}$ denoting Haar measure. Let further $\rho_{N}: \mathfrak{M}_{k} \rightarrow A_{N}$ be the unital inclusion which identifies $a \in \mathfrak{M}_{k}$ with the constant matrix $a \otimes$ $I_{N} \in \mathfrak{M}_{k} \otimes \mathfrak{M}_{N} \simeq \mathfrak{M}_{N k}$. Then, as $N \rightarrow \infty$ the sets $\left\{U_{N}, U_{N}^{-1}\right\}$ and $\rho_{N}\left(\mathfrak{M}_{k}\right)$ are asymptotically free in $\left(A_{N}, \Phi_{N}\right)$.

To deal with the polynomials $\mathcal{Z}\left(B^{d}\right)$ when $B=\mathfrak{M}_{k}$ we will need to identify certain elements in $\mathfrak{M}_{k}^{d}$. If $A \in \mathfrak{M}_{k}$ we shall note by $\varphi_{A} \in$ $\mathfrak{M}_{k}^{d}$ the functional $\varphi_{A}(X)=\operatorname{Tr}\left(X A^{t}\right)$ ( $t$ denotes the transpose). In particular if by $e_{p q}$ we denote the matrix-units in $\mathfrak{M}_{k}$ then we shall also write $\varphi_{p q}$ for $\varphi_{e_{p q}}$.

Theorem. If $B=\mathfrak{M}_{k}$ and $\alpha_{1}, \ldots, \alpha_{m}, \beta_{1}, \ldots, \beta_{n} \in \mathfrak{M}_{k}$ then we have

$$
\begin{aligned}
& \lim _{N \rightarrow \infty} \int_{\mathcal{U}(N ; B)} N^{-1} \operatorname{Tr}\left(z\left(\varphi_{\alpha_{1}}\right)(\omega) \ldots z\left(\varphi_{\alpha_{m}}\right)_{N}(\omega)\left(z\left(\varphi_{\beta_{1}}\right)_{N}(\omega) \ldots z\left(\varphi_{\beta_{n}}\right)_{N}(\omega)\right)^{*} d \mu_{N}(\omega)\right. \\
& = \begin{cases}0 & \text { if } m \neq n \\
\prod_{1 \leq j \leq m} k^{-1} \operatorname{Tr}\left(\alpha_{j} \beta_{j}^{*}\right) & \text { if } m=n .\end{cases}
\end{aligned}
$$

Proof. It is easily seen that it suffices to prove the theorem in case $\alpha_{i}=e_{p_{i} q_{i}}$ and $\beta_{j}=e_{r_{j} s_{j}}$.

Note also that with the notations of the preceding proposition the function

$$
\mathcal{U}(N k) \ni \omega \rightarrow z\left(\varphi_{p q}\right)_{N}(\omega)
$$

can be identified with the first $N \times N$ block of $\rho_{N}\left(e_{1 p}\right) U \rho_{N}\left(e_{q 1}\right)$. Using this observation, the left-hand side of the equality we want to prove becomes

$\lim _{N \rightarrow \infty} k \Phi_{N}\left(\rho_{N}\left(e_{1 p_{1}}\right) U \rho_{N}\left(e_{q_{1} 1}\right) \ldots \rho_{N}\left(e_{1 p_{m}}\right) U \rho_{N}\left(e_{q_{m} 1}\right) \rho_{N}\left(e_{1 s_{n}}\right) U^{*} \rho_{N}\left(e_{r_{n} 1}\right) \ldots \rho_{N}\left(e_{1 s_{1}}\right) U^{*} \rho_{N}\left(e_{r_{1} 1}\right)\right)$.

By the asymptotic freeness recorded in the proposition the limit is equal to

$$
k \Phi\left(U e_{q_{1} p_{2}} U e_{q_{2} p_{3}} \ldots U e_{q_{m} s_{n}} U^{-1} e_{r_{n} s_{n-1}} U^{-1} e_{r_{n-1} s_{n-2}} \ldots U^{-1} e_{r_{1} p_{1}}\right)
$$

where $\Phi$ is the free product trace-state on $C(\mathbb{T}) * \mathfrak{M}_{k}$, where $C(\mathbb{T})$ is given the Haar state and $\mathfrak{M}_{k}$ its unique trace-state. We may now 
invoke the lemma at the beginning of this section to get that this is further equal to 0 if $m \neq n$ and if $m=n$ equal to

$$
\begin{aligned}
& k^{1-(m+1)} \operatorname{Tr} e_{r_{1} p_{1}} \operatorname{Tr} e_{q_{m} s_{m}}\left(\operatorname{Tr} e_{q_{m-1} p_{m}} e_{r_{m} s_{m-1}}\right) \ldots\left(\operatorname{Tr} e_{q_{1} p_{2}} e_{r_{2} s_{1}}\right) \\
& =k^{-m} \delta_{r_{1} p_{1}} \delta_{q_{m} s_{m}}\left(\delta_{q_{m-1} s_{m-1}} \delta_{p_{m} r_{m}}\right) \ldots\left(\delta_{q_{1} s_{1}} \delta_{p_{2} r_{2}}\right) .
\end{aligned}
$$

On the other hand the right-hand side of the equality to be proved is

$$
k^{-m} \prod_{1 \leq j \leq m} \operatorname{Tr} e_{p_{j} q_{j}} e_{s_{j} r_{j}}=k^{-m} \prod_{1 \leq j \leq m} \delta_{p_{j} r_{j}} \delta_{q_{j} s_{j}}
$$

which concludes the proof.

Corollary. Assume $f \in \mathcal{A}\left((1+\varepsilon) \mathcal{D}_{0}\left(\mathfrak{M}_{k}\right)\right)$ for some $\varepsilon>0$ is totally bounded. Then the Taylor expansion of $f$ at the origin is

$$
f=a_{0} \mathbb{1}+\sum_{m \geq 1} \sum_{\substack{1 \leq p_{i}, q_{i} \leq k \\ 1 \leq i \leq m}} a_{m}\left(p_{1}, q_{1} ; \ldots ; p_{m}, q_{m}\right) z\left(\varphi_{p_{1} q_{1}}\right) \ldots z\left(\varphi_{p_{m} q_{m}}\right)
$$

where

$$
a_{0}=\int_{\mathcal{U}\left(N ; \mathfrak{M}_{k}\right)} N^{-1} \operatorname{Tr} f_{N}(\omega) d \mu_{N}(\omega)
$$

for all $N \geq 1$, and where

$$
\begin{aligned}
& a_{m}\left(p_{1}, q_{1} ; \ldots ; p_{m}, q_{m}\right) \\
& =k^{m} \lim _{N \rightarrow \infty} \int_{\mathcal{U}\left(N ; \mathfrak{M}_{k}\right)} N^{-1} \operatorname{Tr} f_{N}(\omega)\left(z\left(\varphi_{p_{1} q_{1}}\right)_{N}(\omega) \ldots z\left(\varphi_{p_{m} q_{m}}\right)_{N}(\omega)\right)^{*} d \mu_{N}(\omega) .
\end{aligned}
$$

In particular we have

$$
\begin{aligned}
& d^{m} f_{N}\left(0_{N}\right)[\beta, \ldots, \beta] \\
& =m ! \sum_{\substack{1 \leq p_{i}, q_{i} \leq 1 \\
1 \leq i \leq m}} a_{m}\left(p_{1}, q_{1} ; \ldots ; p_{m} q_{m}\right) z\left(\varphi_{p_{1} q_{1}}\right)_{N}(\beta) \ldots z\left(\varphi_{p_{m} q_{m}}\right)_{N}(\beta) .
\end{aligned}
$$

Proof. That the Taylor series of $f$ has the form written in the statement of the corollary for some constants $a_{0}, a_{m}(\ldots)$ is the content of 13.8. To check the integral formulae for the coefficients we can reduce the proof to the case when $f$ is given by a polynomial function in $\mathcal{Z}\left(B^{d}\right)$. Indeed by the proof of Proposition 14.2 the sequence of polynomial functions given by the $n$-th order Taylor expansion convergences in the norm $\|\cdot\|_{\infty, 1}$ to $f$ and hence the limits of integrals for these converge to those for $f$. From polynomial functions in $\mathcal{Z}\left(B^{d}\right)$ the proof then reduces to the case when $f$ is $\mathbb{1}$ or some product $z\left(\varphi_{p_{1} q_{1}}\right) \ldots z\left(\varphi_{p_{m} q_{m}}\right)$. 
The statement is then a consequence of the preceding theorem and of the fact that

$$
\int_{\mathcal{U}(N ; B)} z\left(\varphi_{p_{1} q_{1}}\right)(\omega) \ldots z\left(\varphi_{p_{m} q_{m}}\right)(\omega) d \mu_{N}(\omega)=0
$$

which follows immediately from the invariance of the integration under $\omega \rightarrow e^{i \theta} \omega$.

14.4. Totally bounded holomorphic functions on $\mathcal{D}_{0}\left(\mathfrak{M}_{k}\right)$. The norm \|\|$_{\infty, 1}$ of totally bounded functions in $\mathcal{A}\left(\mathcal{D}_{0}\left(\mathfrak{M}_{k}\right)\right)$ is related to a compression of the full free product $C^{*}$-algebra $\mathfrak{M}_{k} *_{\mathbb{C}} C(\mathbb{T})$ (i.e., $\mathfrak{M}_{k}$ and $C(\mathbb{T})$ have the same unit element). More precisely if $e_{i j}, 1 \leq i, j \leq$ $k$ are the matrix units of $\mathfrak{M}_{k} \subset \mathfrak{M}_{k} *_{\mathbb{C}} C(\mathbb{T})$ and $u \in C(\mathbb{T}) \subset \mathfrak{M}_{k} *_{\mathbb{C}} C(\mathbb{T})$ is the unitary element corresponding to the identical function in $C(\mathbb{T})$ we shall work with the $C^{*}$-algebra $B_{k}=e_{11}\left(\mathfrak{M}_{k} *_{\mathbb{C}} C(\mathbb{T})\right) e_{11}$ and use the elements $u_{i j}=e_{1 i} u e_{j 1} \in B_{k}$. The $C^{*}$-algebra $\mathfrak{M}_{k} *_{\mathbb{C}} C(\mathbb{T})$ has sufficiently many finite dimensional representations and representations are in bijection with pairs $(\rho, U)$ where $U$ is a unitary operator and $\rho$ a unital $*$-representation of $\mathfrak{M}_{k}$ on the same Hilbert space (these observations are certainly not new).

Lemma. Let $\beta: \mathcal{Z}\left(\mathfrak{M}_{k}^{d}\right) \rightarrow B_{k}$ be the unital homomorphism so that $\beta\left(\mathcal{Z}\left(\varphi_{i j}\right)\right)=u_{i j}, 1 \leq i, j \leq k$. We have

$$
\|\beta(f)\|=\|f\|_{\infty, 1} \text {. }
$$

Proof. Each element of $\mathcal{U}\left(N ; \mathfrak{M}_{k}\right)$ is a unitary operator on $\mathbb{C}^{N k} \simeq$ $\mathbb{C}^{N} \otimes \mathbb{C}^{k}$ on which we have the representation $T \rightarrow I_{N} \otimes T$ of $\mathfrak{M}_{k}$. Up to unitary equivalence these are the pairs of unitary operator and representation of $\mathfrak{M}_{k}$ for all finite-dimensionaal representations of $\mathfrak{M}_{k} *_{\mathbb{C}}$ $C(\mathbb{T})$. Remark also that if $f \in \mathcal{Z}\left(\mathfrak{M}_{k}^{d}\right)$ and $\omega \in \mathcal{U}\left(N ; \mathfrak{M}_{k}\right)$ then $f_{N}(\omega)$ is just the image of $\beta(f)$ via the representation of $B_{k}$ obtained from restricting the representation of $\mathfrak{M}_{k} * C(\mathbb{T})$ corresponding to $\omega$ to the subalgebra $B_{k}$. Thus

$$
\|\beta(f)\|=\sup _{N} \sup _{\omega \in \mathcal{U}\left(N ; \mathfrak{M}_{k}\right)}\left\|f_{N}(\omega)\right\| .
$$

Since $f_{N}$ is holomorphic

$$
\sup _{\omega \in \mathcal{U}\left(N ; \mathfrak{M}_{k}\right)}\left\|f_{N}(\omega)\right\|=\sup _{\omega \in\left(\mathcal{D}_{0}\left(\mathfrak{M}_{k}\right)\right)_{N}}\left\|f_{N}(\omega)\right\|
$$

and hence $\|\beta(f)\|=\|f\|_{\infty, 1}$. 
Using the proof of Proposition 14.2 it follows that $\beta$ extends by continuity to an isometric homomorphism (which we shall still denote by $\beta)$ of $\mathbb{H}^{\infty}\left((1+\varepsilon) \mathcal{D}_{0}\left(\mathfrak{M}_{k}\right)\right)$ endowed with the \|\|$_{\infty, 1}$-norm into $B_{k}$. If $f \in \mathbb{H}^{\infty}\left(\mathcal{D}_{0}\left(\mathfrak{M}_{k}\right)\right)$ we apply this result to $f(r \cdot) 0<r<1$, to obtain the following proposition.

Proposition. Let $f \in \mathbb{H}^{\infty}\left(\mathcal{D}_{0}\left(\mathfrak{M}_{k}\right)\right)$ have the series expansion

$$
a_{0} \mathbb{1}+\sum_{m \geq 1} \sum_{\substack{1 \leq p_{i}, q_{i} \leq k \\ 1 \leq i \leq m}} a_{m}\left(p_{1}, q_{1} ; \ldots ; p_{m}, q_{m}\right) z\left(\varphi_{p_{1} q_{1}}\right) \ldots z\left(\varphi_{p_{m} q_{m}}\right) .
$$

Then for each $0<r<1$ the series (summation over $m \geq 1$ )

$$
\beta(f(r \cdot))=a_{0} 1+\sum_{m \geq 1} \sum_{\substack{1 \leq p_{i}, q_{i} \leq k \\ 1 \leq i \leq m}} a_{m}\left(p_{1}, q_{1} ; \ldots ; p_{m}, q_{m}\right) u_{p_{1} q_{1}} \ldots u_{p_{m} q_{m}}
$$

is convergent in $B_{k}$ and we have

$$
\|\beta(f(r \cdot))\|=\|f(r \cdot)\|_{\infty, 1}
$$

and

$$
\|f\|_{\infty, 1}=\sup _{0<r<1}\|\beta(f(r \cdot))\|
$$

14.5. Extending the coefficient formula to $\mathbb{H}^{\infty}\left(\mathcal{D}_{0}\left(\mathfrak{M}_{k}\right)\right)$. Some standard arguments about boundary values of bounded holomorphic functions can be used to extend the Corollary of section 14.3 to functions in $\mathbb{H}^{\infty}\left(\mathcal{D}_{0}\left(\mathfrak{M}_{k}\right)\right)$. By basic facts about boundary values for holomorphic functions (see 6.10 in ch. III of [11]) given $f \in \mathbb{H}^{\infty}\left(\mathcal{D}_{0}\left(\mathfrak{M}_{k}\right)\right.$ ) for each $N \geq 1$, there is

$$
\beta_{N}\left(f_{N}\right) \in L^{\infty}\left(\mathcal{U}\left(N ; \mathfrak{M}_{k}\right) ; \mu_{N}\right) \otimes \mathfrak{M}_{N}
$$

where $\mu_{N}$ is Haar measure on $\mathcal{U}\left(N ; \mathfrak{M}_{k}\right) \simeq U(N k)$, so that $\beta_{N}\left(f_{N}\right)(u)=$ $\lim _{r \uparrow 1} f_{N}(r u)$ for $\mu_{N}$-almost all $u \in \mathcal{U}\left(N ; \mathfrak{M}_{k}\right)$.

If $f \in \mathcal{A}\left(t \mathcal{D}_{0}\left(\mathfrak{M}_{k}\right)\right)$ for some $t>0$, it will be convenient to denote by $T_{m}(f)$ and $P_{m}(f)$ the Taylor polynomial of order $m$ of $f$ and respectively its leading term (i.e., $(m !)^{-1}$ times the $m$-th order differential) viewed as elements of $\mathcal{Z}\left(\mathfrak{M}_{k}^{d}\right)$, so that $T_{m}(f)=P_{0}(f)+P_{1}(f)+\cdots+$ $P_{m}(f)$.

Theorem. Assume $f \in \mathbb{H}^{\infty}\left(\mathcal{D}_{0}\left(\mathfrak{M}_{k}\right)\right)$. Then the Taylor expansion of $f$ at the origin is

$$
f=a_{0} \mathbb{1}+\sum_{m \geq 1} \sum_{\substack{1 \leq p_{i}, q_{i} \leq k \\ 1 \leq i \leq m}} a_{m}\left(p_{1}, q_{1} ; \ldots ; p_{m}, q_{m}\right) z\left(\varphi_{p_{1} q_{1}}\right) \ldots z\left(\varphi_{p_{m} q_{m}}\right)
$$


where

$$
a_{0}=\int_{\mathcal{U}\left(N ; \mathfrak{M}_{k}\right)} N^{-1} \operatorname{Tr} \beta_{N}\left(f_{N}\right)(\omega) d \mu_{N}(\omega)
$$

for all $N \geq 1$, and where

$$
\begin{aligned}
& a_{m}\left(p_{1}, q_{1} ; \ldots ; p_{m}, q_{m}\right) \\
& =k^{m} \lim _{N \rightarrow \infty} \int_{\mathcal{U}\left(N ; \mathfrak{M}_{k}\right)} N^{-1} \operatorname{Tr}\left(\beta_{N}\left(f_{N}\right)(\omega)\left(z\left(\varphi_{p_{1} q_{1}}\right)_{N}(\omega) \ldots z\left(\varphi_{p_{m} q_{m}}\right)(\omega)\right)^{*}\right) d \mu_{N}(\omega) .
\end{aligned}
$$

Proof. It will be convenient to use the following notations in this proof:

$$
Q_{N}(\omega)=z\left(\varphi_{p_{1} q_{1}}\right)_{N}(\omega) \ldots z\left(\varphi_{p_{m} q_{m}}\right)(\omega)
$$

and if $F$ is an integrable $\mathfrak{M}_{N^{-}}$valued function on $\mathcal{U}\left(N ; \mathfrak{M}_{k}\right)$

$$
\widetilde{\Phi}_{N}(F(\omega))=N^{-1} \int_{\mathcal{U}\left(N ; \mathfrak{M}_{k}\right)} \operatorname{Tr} F(\omega) d \mu_{N}(\omega) .
$$

We shall apply the corollary in 14.2 to $f(r \cdot) \in \mathbb{H}^{\infty}\left(r^{-1} \mathcal{D}_{0}\left(\mathfrak{M}_{k}\right)\right)$. The Taylor expansion of $f(r \cdot)$ being

$$
a_{0} \mathbb{1}+\sum_{m \geq 1} \sum_{\substack{1 \leq p_{i}, q_{i} \leq k \\ 1 \leq i \leq m}} r^{m} a_{m}\left(p_{1}, q_{1} ; \ldots ; p_{m}, q_{m}\right) z\left(\varphi_{p_{1} q_{1}}\right) \ldots z\left(\varphi_{p_{m} q_{m}}\right)
$$

we have

$$
a_{0}=\widetilde{\Phi}_{N}\left(f_{N}(r \omega)\right)
$$

for all $N \geq 1$ and which as $r \uparrow 1$ gives the formula for $a_{0} \operatorname{using} \beta_{N}\left(f_{N}\right)$.

For $m \geq 1$ we get

$$
r^{m} a_{m}\left(p_{1}, q_{1} ; \ldots ; p_{m} q_{m}\right)=k^{m} \lim _{N \rightarrow \infty} \widetilde{\Phi}_{N}\left(f_{N}(r \omega)\left(Q_{N}(\omega)\right)^{*}\right) .
$$

To prove the theorem we must show that

$$
\lim _{r \uparrow 1} \lim _{N \rightarrow \infty} \widetilde{\Phi}_{N}\left(f_{N}(r \omega)\left(Q_{N}(\omega)\right)^{*}\right)=\lim _{N \rightarrow \infty} \widetilde{\Phi}_{N}\left(\beta_{N}\left(f_{N}\right)(\omega)\left(Q_{N}(\omega)\right)^{*}\right) .
$$

The integral in the left-hand side for fixed $0<r<1$, because of the uniform convergence of the Taylor series equals

$$
\sum_{n \geq 0} \widetilde{\Phi}_{N}\left(P_{n}(f)_{N}(r \omega)\left(Q_{N}(\omega)\right)^{*}\right)
$$

The terms with $n-m \neq 0$ vanish since the integrand is homogeneous of degree $n-m$ with respect to $\omega \rightarrow e^{i \theta} \omega$. Thus the left-hand side equals

$$
\lim _{r \uparrow 1} \lim _{N \rightarrow \infty} \widetilde{\Phi}_{N}\left(P_{m}(f)_{N}(r \omega)\left(Q_{N}(\omega)\right)^{*}\right)
$$




$$
\begin{aligned}
& =\lim _{r \uparrow 1} \lim _{N \rightarrow \infty} r^{m} \widetilde{\Phi}_{N}\left(P_{m}(f)_{N}(\omega)\left(Q_{N}(\omega)\right)^{*}\right) \\
& =\lim _{N \rightarrow \infty} \widetilde{\Phi}_{N}\left(P_{m}(f)_{N}(\omega)\left(Q_{N}(\omega)\right)^{*}\right) .
\end{aligned}
$$

On the other hand using dominated convergence the right-hand side of the equality we want to prove equals

$$
\lim _{N \rightarrow \infty} \lim _{r \uparrow 1} \widetilde{\Phi}_{N}\left(f_{N}(r \omega)\left(Q_{N}(\omega)\right)^{*}\right)
$$

which by the same homogeneity argument used for the left-hand side equals

$$
\begin{aligned}
& \lim _{N \rightarrow \infty} \lim _{r \uparrow 1} \widetilde{\Phi}_{N}\left(P_{m}(f)_{N}(r \omega)\left(Q_{N}(\omega)\right)^{*}\right) \\
& =\lim _{N \rightarrow \infty} \widetilde{\Phi}_{N}\left(P_{m}(f)_{N}(\omega)\left(Q_{N}(\omega)\right)^{*}\right)
\end{aligned}
$$

which concludes the proof.

\section{The Large $N$ Limit of a Totally Bounded Holomorphic Function When $B=\mathfrak{M}_{k}$}

15.1. We will show in this section that there is a large $N$ limit for the boundary values on $\mathcal{U}\left(\mathfrak{M}_{k}\right)$ of a function in $\mathbb{H}^{\infty}\left(\mathcal{D}_{0}\left(\mathfrak{M}_{k}\right)\right)$, which is an operator in a certain subalgebra of a $I I_{1}$-factor. The asymptotic integral formulae for coefficients give rise to formulae involving the large $N$ limit for the coefficients of the function.

15.2. The limit algebras $\mathcal{L}_{k}^{\infty}$ and $\mathcal{H}_{k}^{\infty}$. Let $L^{\infty}(\mathbb{T}) * \mathfrak{M}_{k}$ be the von Neumann algebra with the free-product trace-state $\mu * k^{-1} \mathrm{Tr}$, where $\mu$ is Haar measure and let $U$ be the Haar unitary arising from the identical function in $L^{\infty}(\mathbb{T})$ and consider the matrix units $e_{i j}$ from $\mathfrak{M}_{k}, 1 \leq i, j \leq k$, viewed as elements of the free product. We define $\mathcal{L}_{k}^{\infty}$ to be the compression $e_{11}\left(L^{\infty}(\mathbb{T}) * \mathfrak{M}_{k}\right) e_{11}$ which is generated by the elements $v_{i j}=e_{1 i} U e_{j 1}, 1 \leq i, j \leq k$. With respect to the trace-state $\psi$ on $\mathcal{L}_{k}^{\infty}$ (which is a $I I_{1}$-factor), we have that the elements $\left\{v_{p_{1} q_{1}} \ldots v_{p_{m} q_{m}} \mid m \geq 1,1 \leq p_{j}, q_{j} \leq k, 1 \leq j \leq m\right\}$ form an orthogonal family in $L^{2}\left(\mathcal{L}_{k}^{\infty}, \psi\right)$ and $\left|v_{p_{1} q_{1}} \ldots v_{p_{m} q_{m}}\right|_{2}=k^{-m / 2}$ (an immediate consequence of the lemma in 14.3).

We define $\mathcal{H}_{k}^{\infty}$ to be the weakly closed subalgebra of $\mathcal{L}_{k}^{\infty}$ generated by the $v_{i j} 1 \leq i, j \leq k$ (the weak topology is with respect to the standard form of $\left.\mathcal{L}_{k}^{\infty}\right)$.

Since Haar measure $\mu$ on $\mathbb{T}$ is invariant under rotations we get that these yield automorphisms of the free product $L^{\infty}(\mathbb{T}) * \mathfrak{M}_{k}$ which act as the identity on $\mathfrak{M}_{k}$. We infer the existence of automorphisms $\alpha\left(e^{i \theta}\right)$ of 
$\mathcal{L}_{k}^{\infty}$ so that $\alpha\left(e^{i \theta}\right)\left(v_{p q}\right)=e^{i \theta} v_{p q}$ for $1 \leq p, q \leq k$. Also $\mathcal{H}_{k}^{\infty}$ is invariant under the $\alpha\left(e^{i \theta}\right)$.

For $0 \leq r<1$ let $P_{r}\left(e^{i \theta}\right)=\left(1-r^{2}\right)\left|e^{i \theta}-r\right|^{-2}$ be the Poisson kernel and if $x \in L^{2}\left(\mathcal{L}_{k}^{\infty}, \psi\right)$ let

$$
\gamma(r)(x)=(2 \pi)^{-1} \int_{0}^{2 \pi} P_{r}\left(e^{i \theta}\right) \alpha\left(e^{i \theta}\right)(x) d \theta
$$

which is defined in the $L^{2}$-sense. Then $\gamma(r)$ is an ultra-weakly continuous unital completely positive and completely contractive map $\gamma(r)$ : $\mathcal{L}_{k}^{\infty} \rightarrow \mathcal{L}_{k}^{\infty}$. It is also easily seen that $\gamma(r)$ leaves $\mathcal{H}_{k}^{\infty}$ invariant and that $\gamma(r)\left(v_{p_{1} q_{1}} \ldots v_{p_{m} q_{m}}\right)=r^{m} v_{p_{1} q_{1}} \ldots v_{p_{m} q_{m}}$. Moreover $\lim _{r \uparrow 1} \mid \gamma(r)(x)-$ $\left.x\right|_{2}=0$ for all $x \in L^{2}\left(\mathcal{L}_{k}^{\infty}, \psi\right)$.

15.3. The large $N$ limit map $\beta_{\infty}$. The construction of the large $N$ limit homomorphism $\beta_{\infty}: \mathbb{H}^{\infty}\left(\mathcal{D}_{0}\left(\mathfrak{M}_{k}\right)\right) \rightarrow \mathcal{H}_{k}^{\infty}$ will be done in several steps.

Step 1. On $\mathcal{Z}\left(\mathfrak{M}_{k}^{d}\right)$ we define $\beta_{\infty}$ as the linear map such that

$$
\beta_{\infty}\left(z\left(\varphi_{p_{1} q_{1}}\right) \ldots z\left(\varphi_{p_{m} q_{m}}\right)\right)=v_{p_{1} q_{1}} \ldots v_{p_{m} q_{m}}
$$

and $\beta_{\infty}(\mathbb{1})=1$. From the large $N$ limit result in the proposition of 14.3 it follows easily that $\left\|\beta_{\infty}(f)\right\| \leq\|f\|_{\infty, 1}$ for $f \in \mathcal{Z}\left(\mathfrak{M}_{k}^{d}\right)$.

Step 2. Since $\beta_{\infty}$ is contractive we can extend its definition to a contractive homomorphism of the closure of $\mathcal{Z}\left(\mathfrak{M}_{k}^{d}\right)$ in $\|\cdot\|_{\infty, 1}$-norm. This Banach subalgebra of $\mathbb{H}^{\infty}\left(\mathcal{D}_{0}\left(\mathfrak{M}_{k}\right)\right)$ contains in particular all $f(r \cdot)$ where $f \in \mathbb{H}^{\infty}\left(\mathcal{D}_{0}\left(\mathfrak{M}_{k}\right)\right)$ and $0<r<1$ in view of the proof of the proposition in 14.2 .

Step 3. If $f \in \mathcal{Z}\left(\mathfrak{M}_{k}^{d}\right)$ and if

$$
a_{0} \mathbb{1}+\sum_{m \geq 1} \sum_{\substack{1 \leq p_{i}, q_{i} \leq k \\ 1 \leq i \leq m}} a_{m}\left(p_{1}, q_{1} ; \ldots ; p_{m}, q_{m}\right) z\left(\varphi_{p_{1} q_{1}}\right) \ldots z\left(\varphi_{p_{m} q_{m}}\right)
$$

is its (finite) series expansion then

$$
\begin{aligned}
& \left|a_{0}\right|^{2}+\sum_{m \geq 1} \sum_{\substack{1 \leq p_{i}, q_{i} \leq k \\
1 \leq i \leq m}}\left|a_{m}\left(p_{1}, q_{1} ; \ldots ; p_{m}, q_{m}\right)\right|^{2} k^{-m} \\
& =\left|\beta_{\infty}(f)\right|_{2}^{2} \leq\left\|\beta_{\infty}(f)\right\|^{2} \leq\|f\|_{\infty, 1} .
\end{aligned}
$$

Hence, more generally, if $f$ is in the \|\|$_{\infty, 1}$ closure of $\mathcal{Z}\left(\mathfrak{M}_{k}^{d}\right)$ we have

$$
\beta_{\infty}(f)=a_{0} 1+\sum_{m \geq 1} \sum_{\substack{1 \leq p_{i}, q_{i} \leq k \\ 1 \leq i \leq m}} a_{m}\left(p_{1}, q_{1} ; \ldots ; p_{m}, q_{m}\right) v_{p_{1}, q_{1}} \ldots v_{p_{m}, q_{m}}
$$


where the series converges in ||$_{2}$-norm and

$$
\left|a_{0}\right|^{2}+\sum_{m \geq 1} \sum_{\substack{1 \leq p_{i}, q_{i} \leq k \\ 1 \leq i \leq m}}\left|a_{m}\left(p_{1}, q_{1} ; \ldots ; p_{m}, q_{m}\right)\right|^{2} k^{-m} \leq\|f\|_{\infty, 1}^{2} .
$$

Step 4. If $f \in \mathbb{H}^{\infty}\left(\mathfrak{M}_{k}^{d}\right)$ we can apply the result of Step 3 to the $f(r \cdot)$ for $0<r<1$ and we get that

$$
\|f\|_{1, \infty}^{2} \geq\|f(r \cdot)\|_{\infty, 1}^{2} \geq\left|a_{0}\right|^{2}+\sum_{m \geq 1} \sum_{\substack{1 \leq p_{i}, q_{i} \leq k \\ 1 \leq i \leq m}}\left|a_{m}\left(p_{1}, q_{1} ; \ldots ; p_{m}, q_{m}\right)\right|^{2} r^{2 m} k^{-m}
$$

Hence, letting $r \uparrow 1$ we find that

$$
\|f\|_{1, \infty}^{2} \geq\left|a_{0}\right|^{2}+\sum_{m \geq 1} \sum_{\substack{1 \leq p_{i}, q_{i} \leq k \\ 1 \leq i \leq m}}\left|a_{m}\left(p_{1}, q_{1} ; \ldots ; p_{m}, q_{m}\right)\right|^{2} k^{-m} .
$$

Step 5. We define $\beta_{\infty}(f) \in L^{2}\left(\mathcal{L}_{k} ; \psi\right)$ for $f \in \mathbb{H}^{\infty}\left(\mathcal{D}_{0}\left(\mathfrak{M}_{k}\right)\right)$, using the result of Step 4, to be

$$
\beta_{\infty}(f)=a_{0} 1+\sum_{m \geq 1} \sum_{\substack{1 \leq p_{i}, q_{i} \leq k \\ 1 \leq i \leq m}} a_{m}\left(p_{1}, q_{1} ; \ldots ; p_{m}, q_{m}\right) v_{p_{1} q_{1}} \ldots v_{p_{m} q_{m}}
$$

which coincides on the \|\|$_{\infty, 1}$ closure $\mathcal{Z}\left(\mathfrak{M}_{k}^{d}\right)$ with the $\beta_{\infty}$ already constructed. Moreover $\|f\|_{\infty, 1} \geq\left|\beta_{\infty}(f)\right|_{2}$ for general $f$. Remark that with this definition

$$
\left|\beta_{\infty}(f)-\beta_{\infty}(f(r \cdot))\right|_{2} \rightarrow 0
$$

as $r \uparrow 1$ and since

$$
\left\|\beta_{\infty}(f(r \cdot))\right\| \leq\|f(r \cdot)\|_{1, \infty} \leq\|f\|_{\infty, 1}
$$

we infer that $\left\|\beta_{\infty}(f)\right\| \leq\|f\|_{\infty, 1}$ and $\beta_{\infty}(f(r \cdot))$ converges to $\beta_{\infty}(f)$ *-strongly and hence is in $\mathcal{H}_{k}^{\infty}$.

We will record our conclusions as the following proposition.

Proposition. If $f \in \mathbb{H}^{\infty}\left(\mathcal{D}_{0}\left(\mathfrak{M}_{k}\right)\right)$ then $\beta_{\infty}(f)$ is defined by the series convergent in ||$_{2}$-norm

$$
\beta_{\infty}(f)=a_{0} 1+\sum_{m \geq 1} \sum_{\substack{1 \leq p_{i}, q_{i} \leq k \\ 1 \leq i \leq m}} a_{m}\left(p_{1}, q_{1} ; \ldots ; p_{m}, q_{m}\right) v_{p_{1} q_{1}} \ldots v_{p_{m} q_{m}}
$$

and we have $\beta_{\infty}(f) \in \mathcal{H}_{k}^{\infty}$ and $\left\|\beta_{\infty}(f)\right\| \leq\|f\|_{\infty, 1}$. Moreover

$$
\mid \beta_{\infty}(f)-\beta_{\infty}(f(r \cdot)) \|_{2} \rightarrow 0
$$

as $r \rightarrow \infty$ and $\beta_{\infty}$ is a homomorphism of Banach algebras. 
Proof. The only assertion we still must prove is that $\beta_{\infty}$ is multiplicative. This is clear on $\mathcal{Z}\left(\mathfrak{M}_{k}^{d}\right)$ by the definition and hence also on its \|\|$_{\infty, 1}$-closure. Thus we have

$$
\beta_{\infty}((f g)(r \cdot))=\beta_{\infty}(f(r \cdot)) \beta_{\infty}(g(r \cdot))
$$

and for $r \uparrow 1$ the equality converges strongly to

$$
\beta_{\infty}(f g)=\beta_{\infty}(f) \beta_{\infty}(g)
$$

Corollary. If $f \in \mathbb{H}^{\infty}\left(\mathcal{D}_{0}\left(\mathfrak{M}_{k}\right)\right)$ then we have $a_{0}=\psi\left(\beta_{\infty}(f)\right)$ and

$$
k^{-m} a_{m}\left(p_{1}, q_{1} ; \ldots ; p_{m}, q_{m}\right)=\psi\left(\beta_{\infty}(f) v_{p_{m} q_{m}}^{*} \ldots v_{p_{1} q_{1}}^{*}\right) .
$$

15.4. Remark. It is a natural question whether the formal expression

$$
1 \otimes 1+\sum_{m \geq 1} \sum_{\substack{1 \leq p_{i}, q_{i} \leq k \\ 1 \leq i \leq m}} k^{m} v_{p_{1} q_{1}} \ldots v_{p_{m} q_{m}} \otimes v_{p_{m} q_{m}}^{*} \ldots v_{p_{2} q_{2}}^{*}
$$

which plays the role of the Cauchy kernel of the large $N$ limit gives rise to an unbounded affiliated operator of the von Neumann algebra $\mathcal{L}_{k}^{\infty} \otimes \mathcal{L}_{k}^{\infty}$ or perhaps $\mathcal{L}_{k}^{\infty} \otimes\left(\mathcal{L}_{k}^{\infty}\right)^{o p}$ (op denotes the opposite algebra). It is also not clear whether there is a chance that the formal kernel

$$
\mathbb{1} \otimes 1+\sum_{m \geq 1} \sum_{\substack{1 \leq p_{i}, q_{i} \leq k \\ 1 \leq i \leq m}} z\left(\varphi_{p_{1} q_{1}}\right) \ldots z\left(\varphi_{p_{m} q_{m}}\right) \otimes v_{p_{m} q_{m}}^{*} \ldots v_{p_{1} q_{1}}^{*}
$$

exhibits some good analytic properties. It is also natural to ask whether some group object is involved here, possibly a free quantum group (see [3] and references therein).

\section{The Asymptotic Integral Formula and the Large $N$ Limit IN THE UNIT Disk WHEN $B=\mathbb{C}^{k}$}

16.1. Here we will work out the analogue of the results of sections 14 and 15 in the case of the commutative $C^{*}$-algebra $\mathbb{C}^{k}$, which is at times somewhat simpler. Often the arguments will be quite similar to those for $B=\mathfrak{M}_{k}$ and our presentation will be more compressed. Note that when $B=\mathbb{C}^{k}$ the components of the stably matricial unitary group are $\mathcal{U}\left(N ; \mathbb{C}^{k}\right) \simeq U(N)^{k}$. 
16.2. The series expansion when $B=\mathbb{C}^{k}$. The natural basis in $B^{d}$ when $B=\mathbb{C}^{k}$ is $\varphi_{1}, \ldots, \varphi_{k}$, where $\varphi_{j}\left(w_{1}, \ldots, w_{k}\right)=w_{j}, 1 \leq j \leq k$ and the Taylor series for a fully matricial holomorphic function can be written

$$
f_{1}(0) \mathbb{1}+\sum_{m \geq 1} \sum_{\substack{1 \leq j_{p} \leq k \\ 1 \leq p \leq m}} a\left(j_{1}, \ldots, j_{m}\right) z\left(\varphi_{j_{1}}\right) \ldots z\left(\varphi_{j_{n}}\right) .
$$

Note that an element $\beta \in \mathfrak{M}_{N}\left(\mathbb{C}^{k}\right)$ identifies with a $k$-tuple $\beta=$ $\left(\beta_{1}, \ldots, \beta_{k}\right) \in\left(\mathfrak{M}_{N}(\mathbb{C})\right)^{k}$ and $z\left(\varphi_{j_{1}}\right)_{N}(\beta) \ldots z\left(\varphi_{j_{m}}\right)_{N}(\beta)=\beta_{j_{1}} \beta_{j_{2}} \ldots \beta_{j_{m}}$.

16.3. The asymptotic integral formula for the coefficients. The asymptotic integral formula for the coefficients is a consequence of our basic asymptotic freeness result for random Haar unitary matrices [12]. We record the result as the next lemma.

Lemma ([12]). Let $U_{1, N}, \ldots, U_{k, N}$ be the $k$ projection functions on $U(N)^{k} \simeq \mathcal{U}\left(N ; \mathbb{C}^{k}\right)$ with values in $\mathfrak{M}_{N}$ viewed as elements of the noncommutative probability space $\left(A_{N}, \Phi_{N}\right)$, where $A_{N}=\mathfrak{M}_{N}\left(L^{\infty}\left(U(N)^{k} ; d \mu_{N}\right)\right)$

and where

$$
\Phi_{N}(T)=\int_{U(N)^{k}} N^{-1} \operatorname{Tr} T d \mu_{N}
$$

with $\mu_{N}$ denoting Haar measure. Then $\left\{U_{1 N}, U_{1, N}^{-1}\right\}, \ldots,\left\{U_{k N}, U_{k N}^{-1}\right\}$ are asymptotically free as $N \rightarrow \infty$ in $\left(A_{N}, \Phi_{N}\right)$.

Remarking that $U_{j, N}=z\left(\varphi_{j}\right)_{N}$ this immediately implies the asymptotic integral formula for coefficients. Indeed in the large $N$ limit the $U_{j, N}$ behave like the generating unitaries in a free group algebra with respect to the von Neumann trace.

Proposition. If $B=\mathbb{C}^{k}$ and $i_{1}, \ldots, i_{m}, j_{1}, \ldots, j_{n} \in\{1, \ldots, k\}$ then

$$
\begin{aligned}
& \lim _{N \rightarrow \infty} \int_{\mathcal{U}(N ; B)} N^{-1} \operatorname{Tr}\left(z\left(\varphi_{i_{1}}\right)_{N}(\omega) \ldots z\left(\varphi_{i_{m}}\right)_{N}(\omega)\left(z\left(\varphi_{j_{1}}\right)_{N}(\omega) \ldots z\left(\varphi_{j_{n}}\right)_{N}(\omega)\right)^{*}\right) d \mu_{N}(\omega) \\
& = \begin{cases}0 & \text { if } m \neq n \\
\delta_{i_{1} j_{1}} \ldots \delta_{i_{m} j_{m}} & \text { if } m=n .\end{cases}
\end{aligned}
$$

If $n=0$ and $m>0$ the above integral is zero.

The same argument as in the proof of Corollary 14.3 then yields the following corollary. 
Corollary. Assume $f \in \mathbb{H}^{\infty}\left((1+\varepsilon) \mathcal{D}_{0}\left(\mathbb{C}^{k}\right)\right)$ for some $\varepsilon>0$. Then the Taylor expansion of $f$ at the origin is

$$
f=a_{0} \mathbb{1}+\sum_{m \geq 1} \sum_{\substack{1 \leq j_{p} \leq k \\ 1 \leq p \leq m}} a\left(j_{1}, \ldots, j_{m}\right) z\left(\varphi_{j_{1}}\right) \ldots z\left(\varphi_{j_{m}}\right)
$$

where

$$
a_{0}=\int_{\mathcal{U}\left(N ; \mathbb{C}^{k}\right)} N^{-1} \operatorname{Tr} f_{N}(\omega) d \mu_{N}(\omega)
$$

for all $N \geq 1$, and where

$$
\begin{aligned}
& a\left(j_{1}, \ldots, j_{m}\right) \\
& =\lim _{N \rightarrow \infty} \int_{\mathcal{U}\left(N ; \mathbb{C}^{k}\right)} N^{-1} \operatorname{Tr} f_{N}(\omega)\left(z\left(\varphi_{j_{1}}\right)_{N}(\omega) \ldots z\left(\varphi_{j_{m}}\right)_{N}(\omega)\right)^{*} d \mu_{N}(\omega) .
\end{aligned}
$$

16.4. Totally bounded holomorphic functions on $\mathcal{D}_{0}\left(\mathbb{C}^{k}\right)$. In case $B=\mathbb{C}^{k}$ the norm \|\|$_{\infty, 1}$ and totally bounded functions in $\mathcal{A}\left(\mathcal{D}_{0}\left(\mathbb{C}^{k}\right)\right)$ are connected with the full $C^{*}$-algebra of the free group on $k$ generators $F_{k}$. We shall denote by $u_{1}, \ldots, u_{k}$ the $k$ generating unitary elements of the full $C^{*}$-algebra of $F_{k} C^{*}\left(F_{k}\right)$. Unital representations of $C^{*}\left(F_{k}\right)$ are in bijection with unitary representations of $F_{k}$ which in turn are in bijection with $k$-tuples of unitary operators on Hilbert spaces and $C^{*}\left(F_{k}\right)$ has sufficiently many finite-dimensional representations. This immediately implies the following lemma.

Lemma. Let $\beta: \mathcal{Z}\left(\left(\mathbb{C}^{k}\right)^{d}\right) \rightarrow C^{*}\left(F_{k}\right)$ be the unital homomorphism so that $\beta\left(z\left(\varphi_{j}\right)\right)=u_{j}, 1 \leq j \leq k$. We have

$$
\|\beta(f)\|=\|f\|_{\infty, 1} \text {. }
$$

Using the proof of Proposition 14.2 it follows that $\beta$ extend by continuity to an isometric homomorphism (which we shall still denote by $\beta$ )of $\mathbb{H}^{\infty}\left((1+\varepsilon) \mathcal{D}_{0}\left(\mathbb{C}^{k}\right)\right)$ endowed with the \|\|$_{\infty, 1}$-norm into $C^{*}\left(F_{k}\right)$. If $f \in \mathbb{H}^{\infty}\left(\mathcal{D}_{0}\left(\mathbb{C}^{k}\right)\right)$ we apply this result to $f(r \cdot), 0<r<1$, to obtain the following proposition.

Proposition. Let $f \in \mathbb{H}^{\infty}\left(\mathcal{D}_{0}\left(\mathbb{C}^{k}\right)\right)$ have the series expansion

$$
a_{0} \mathbb{1}+\sum_{m \geq 1} \sum_{\substack{1 \leq j_{p} \leq k \\ 1 \leq p \leq m}} a\left(j_{1}, \ldots, j_{m}\right) z\left(\varphi_{j_{1}}\right) \ldots z\left(\varphi_{j_{m}}\right) .
$$


Then for each $0<r<1$ the series (summation over $m \geq 1$ )

$$
\beta(f(r \cdot))=a_{0} 1+\sum_{m \geq 1}\left(\sum_{\substack{1 \leq j_{p} \leq k \\ 1 \leq p \leq m}} r^{m} a\left(j_{1}, \ldots, j_{m}\right) u_{j_{1}} \ldots u_{j_{m}}\right)
$$

is convergent in $C^{*}\left(F_{k}\right)$ and we have

$$
\|\beta(f(r \cdot))\|=\|f(r \cdot)\|_{1, \infty}
$$

and

$$
\|f\|_{\infty, 1}=\sup _{0<r<1}\|\beta(f(r \cdot))\|
$$

It is also possible to strengthen the asymptotic integral formula to $\mathbb{H}^{\infty}\left(\mathcal{D}_{0}\left(\mathbb{C}^{k}\right)\right)$. Like in the case of $\mathfrak{M}_{k}$ also for $\mathbb{C}^{k}$ there is a boundary value map $\beta_{N}\left(f_{N}\right)(u)=\lim _{r \uparrow 1} f_{N}(r u)$ for $\mu_{N}$-almost all $u \in$ $\mathcal{U}\left(N ; \mathbb{C}^{k}\right) \simeq(U(N))^{k}$ and $\beta_{N}\left(f_{N}\right) \in \mathfrak{M}_{N}\left(L^{\infty}\left(\mathcal{U}\left(N ; \mathbb{C}^{k}\right) ; d \mu_{N}\right)\right)$ (see ch. III of [11]).

Theorem. Assume $f \in \mathbb{H}^{\infty}\left(\mathcal{D}_{0}\left(\mathbb{C}^{k}\right)\right)$. Then the Taylor expansion of $f$ at the origin is

$$
f=a_{0} \mathbb{1}+\sum_{m \geq 1} \sum_{\substack{1 \leq j_{p} \leq k \\ 1 \leq p \leq m}} a\left(j_{1}, \ldots, j_{m}\right) z\left(\varphi_{j_{1}}\right) \ldots z\left(\varphi_{j_{m}}\right)
$$

where

$$
a_{0}=\int_{\mathcal{U}\left(N ; \mathbb{C}^{k}\right)} N^{-1} \operatorname{Tr} \beta_{N}\left(f_{N}\right)(\omega) d \mu_{N}(\omega)
$$

for all $N \geq 1$, and where

$$
\begin{aligned}
& a\left(j_{1}, \ldots, j_{m}\right) \\
& =\lim _{N \rightarrow \infty} \int_{\mathcal{U}\left(N ; \mathbb{C}^{k}\right)} N^{-1} \operatorname{Tr}\left(\beta_{N}\left(f_{N}\right)\left(z\left(\varphi_{j_{1}}\right)_{N}(\omega) \ldots z\left(\varphi_{j_{m}}\right)_{N}(\omega)\right)^{*} d \mu_{N}(\omega)\right.
\end{aligned}
$$

The proof is along the same lines as in the case of $\mathfrak{M}_{k}$ and will be omitted. 
16.5. The large $N$ limit homomorphism $\beta_{\infty}$ when $B=\mathbb{C}^{k}$. In case $B=\mathbb{C}^{k}$ the role of $\mathcal{L}_{k}^{\infty}, \mathcal{H}_{k}^{\infty}$ will be played by the free group $I I_{1^{-}}$ factor $L\left(F_{k}\right)$ and by $H\left(F_{k}\right)$ the weakly closed nonselfadjoint subalgebra of $L\left(F_{k}\right)$ generated by 1 and the unitary operators $\lambda\left(g_{1}\right), \ldots, \lambda\left(g_{k}\right)$ corresponding to the generators of $F_{k}$. Also here there is an action of $\mathbb{T}$ by automorphisms of $\alpha\left(e^{i \theta}\right)$ of $L\left(F_{k}\right)$ where $\alpha\left(e^{i \theta}\right) \lambda\left(g_{j}\right)=e^{i \theta} \lambda\left(g_{j}\right)$ and this gives rise to the semigroup of completely positive maps $\gamma(r)$, $0 \leq r \leq 1$, where

$$
\gamma(r)(x)=(2 \pi)^{-1} \int_{0}^{2 \pi} P_{r}\left(e^{i \theta}\right) \alpha\left(e^{i \theta}\right)(x) d \theta
$$

with $P_{r}\left(e^{i \theta}\right)=\left(1-r^{2}\right)\left|e^{i \theta}-r\right|^{-2}$. Then $\gamma(r)\left(\lambda\left(g_{i_{1}}\right) \ldots \lambda\left(g_{i_{m}}\right)\right)=$ $r^{m} \lambda\left(g_{i_{1}}\right) \ldots \lambda\left(g_{i_{m}}\right)$.

The construction of the homomorphism $\beta_{\infty}: \mathbb{H}^{\infty}\left(\mathcal{D}_{0}\left(\mathbb{C}^{k}\right)\right) \rightarrow H\left(F_{k}\right)$ is also similar to the construction in case $B=\mathfrak{M}_{k}$.

Step 1. On $\mathcal{Z}\left(\left(\mathbb{C}^{k}\right)^{d}\right)$ we define $\beta_{\infty}$ as the linear map so that $\beta_{\infty}(\mathbb{1})=$ 1 and $\beta_{\infty}\left(z\left(\varphi_{j_{1}}\right) \ldots z\left(\varphi_{j_{m}}\right)\right)=\lambda\left(g_{j_{1}}\right) \ldots \lambda\left(g_{j_{m}}\right)$. Comparing with the lemma and proposition in 16.3, we see that $\beta_{\infty}$ arises from $\beta$ via the homomorphism $C^{*}\left(F_{k}\right) \rightarrow L\left(F_{k}\right)$ which passes via the reduced $C^{*}$ algebra and as such $\left\|\beta_{\infty}(f)\right\| \leq\|f\|_{\infty, 1}$ for $f \in \mathcal{Z}\left(\left(\mathbb{C}^{k}\right)^{d}\right)$ and this extends by continuity to the norm-closure of $\mathcal{Z}\left(\left(\mathbb{C}^{k}\right)^{d}\right)$ in \|\|$_{\infty, 1}$-norm. This shows in particular that if $f \in \mathbb{H}^{\infty}\left(\mathcal{D}_{0}\left(\mathbb{C}^{k}\right)\right)$ then $\beta_{\infty}(f(r \cdot))$ is defined for $0<r<1$ and $\left\|\beta_{\infty}(f(r \cdot))\right\| \leq\|f(r \cdot)\|_{\infty, 1}$.

Step 2. Like in the case of $\mathfrak{M}_{k}$ also in the case of $\mathbb{C}^{k}$ if $f \in \mathbb{H}^{\infty}\left(\mathcal{D}_{0}\left(\mathbb{C}^{k}\right)\right)$ has series expansion

$$
a_{0} \mathbb{1}+\sum_{m \geq 1} \sum_{\substack{1 \leq j_{p} \leq k \\ 1 \leq p \leq m}} a\left(j_{1}, \ldots, j_{m}\right) z\left(\varphi_{j_{1}}\right) \ldots z\left(j_{m}\right)
$$

we find that

$$
\left|a_{0}\right|^{2}+\sum_{m \geq 1} \sum_{\substack{1 \leq j_{p} \leq k \\ 1 \leq p \leq m}}\left|a\left(j_{1}, \ldots, j_{m}\right)\right|^{2} \leq\|f\|_{\infty, 1}^{2} .
$$

This is first shown when the sum is finite, then for $f(r \cdot)$ and then in full generality.

Step 3. If $f \in \mathbb{H}^{\infty}\left(\mathcal{D}_{0}\left(\mathbb{C}^{k}\right)\right)$ we define $\beta_{\infty}(f)$ as an element of $L^{2}\left(L\left(F_{k}\right), \tau\right)$ ( $\tau$ the unique trace-state) by

$$
\beta_{\infty}(f)=a_{0} 1+\sum_{m \geq 1} \sum_{\substack{1 \leq j_{p} \leq k \\ 1 \leq p \leq m}} a\left(j_{1}, \ldots, j_{m}\right) \lambda\left(g_{j_{1}}\right) \ldots \lambda\left(g_{j_{m}}\right) .
$$


We have $\lim _{r \uparrow 1}\left|\beta_{\infty}(f)-\beta_{\infty}(f(r \cdot))\right|_{2}=0$ and $\sup _{0<r<1}\left\|\beta_{\infty}(f(r \cdot))\right\| \leq$ $\|f\|_{\infty, 1}$ from which we infer that $\beta_{\infty}(f)$ is bounded and $\left\|\beta_{\infty}(f)\right\| \leq$ $\|f\|_{\infty, 1}$ and $\beta_{\infty}(f)$ is the $*$-strong limit of $\beta_{\infty}(f(r \cdot))$ as $r \uparrow 1$.

We conclude that $\beta_{\infty}$ has properties similar to those in the case of $\mathfrak{M}_{k}$.

Proposition. If $f \in \mathbb{H}^{\infty}\left(\mathcal{D}_{0}\left(\mathbb{C}^{k}\right)\right)$ then $\beta_{\infty}(f)$ is defined by the series convergent in ||$_{2}$-norm

$$
\beta_{\infty}(f)=a_{0} 1+\sum_{m \geq 1} \sum_{\substack{1 \leq j_{p} \leq k \\ 1 \leq p \leq m}} a\left(j_{1}, \ldots, j_{p}\right) \lambda\left(g_{j_{1}}\right) \ldots \lambda\left(g_{j_{m}}\right)
$$

and we have $\beta_{\infty}(f) \in H\left(F_{k}\right)$ and $\left|\beta_{\infty}(f)-\beta_{\infty}(f(r \cdot))\right|_{2} \rightarrow 0$ as $r \rightarrow$ $\infty$ and $\beta_{\infty}$ is a homomorphism of Banach algebras. Moreover $a_{0}=$ $\tau\left(\beta_{\infty}(f)\right)$ and $a\left(j_{1}, \ldots, j_{m}\right)=\tau\left(\beta_{\infty}(f) \lambda\left(g_{j_{m}}^{-1}\right) \ldots \lambda\left(g_{j_{1}}^{-1}\right)\right)$.

\section{Unbounded Fully Matricial Analytic Functions}

17.1. We saw in the preceding sections that totally bounded fully matricial analytic functions behave well with respect to the large $N$ limit. In this section we show that without the totally boundedness assumption the components of a fully matricial analytic function may increase wildly.

Theorem. Let $B$ be a finite-dimensional $C^{*}$-algebra with $\operatorname{dim} B>1$. Then there is $f=\left(f_{k}\right)_{k \geq 1} \in \mathcal{A}(\mathfrak{M}(B))$ so that each $f_{k}$ is a polynomial function and $\|f\|_{\infty, R}=\infty$ for all $R>0$.

Underlying the proof of the theorem is the following lemma.

Lemma. Let $B$ be a finite-dimensional $C^{*}$-algebra, with $\operatorname{dim} B>1$ and let $\varphi_{1}, \ldots, \varphi_{n}$ be a basis of $B^{d}$. Let $m_{j}=z\left(\varphi_{\alpha(1, j)}\right) \ldots z\left(\varphi_{\alpha(N, j)}\right) \in$ $\mathcal{Z}\left(B^{d}\right), 1 \leq j \leq p$ be monomials of the same degree $N$ and which are algebraically free in the free semigroup of all monomials in $z\left(\varphi_{1}\right), \ldots, z\left(\varphi_{n}\right)$ and let $g=\sum_{\sigma \in \mathfrak{S}_{p}} \operatorname{sign}(\sigma) m_{\sigma(1)} \ldots m_{\sigma(p)}$ be the total antisymmetrization of $m_{1} \ldots m_{p}$. If $g=\left(g_{k}\right)_{k \geq 1}$, then each $g_{k}$ is a polynomial function and $g_{k} \equiv 0$ if $k^{2}<p$, but there is some $N$ such that $g_{N}$ is not identically zero.

Proof of the lemma. If $\beta \in \mathfrak{M}_{k}(B)$ then $g_{k}(\beta)$ is the total antisymmetrization of the product of $p$ matrices of size $k \times k$. Since the vector 
space of $k \times k$ matrices has dimension $k^{2}$, there are no non-zero antisymmetric $p$-tensors in $\underbrace{\mathfrak{M}_{k} \otimes \cdots \otimes \mathfrak{M}_{k}}_{p \text {-times }}$ if $p>k^{2}$. On the other hand $\mathcal{Z}\left(B^{d}\right)$ is a free algebra with generators $z\left(\varphi_{1}\right), \ldots, z\left(\varphi_{d}\right)$ so $g$ is nonzero in $\mathcal{Z}\left(B^{d}\right) \subset \mathcal{A}(\mathfrak{M}(B))$ and hence for some $N, g_{N}$ is not identically zero.

Proof of the theorem. Using the lemma, we can find a sequence $1<N_{1}<N_{2}<\ldots$ so that $N_{j+1}>N_{j+1}^{2}$ for all $j \in \mathbb{N}$ and $g_{j}=$ $\left(g_{j k}\right)_{k \geq 1} \in \mathcal{Z}\left(B^{d}\right) \subset \mathcal{A}(\mathfrak{M}(B))$ so that $k \leq N_{j} \Rightarrow g_{j k} \equiv 0$ and $g_{j} N_{j+1}$ is not identically zero ( $g_{j}$ is an $N_{j+1}^{2}$-antisymmetrization). Recursively, replacing $g_{j}$ by some multiple $\lambda_{j} g_{j}, \lambda_{j} \in \mathbb{C}$ we can make sure that

$$
\sup _{\substack{\beta \in \mathfrak{M}_{N_{j+1}(B)} \\\|\beta\|<1 / j}}\left\|\left(g_{1 N_{j+1}}+\cdots+g_{j N_{j+1}}\right)(\beta)\right\|>j .
$$

Note that $g_{p k} \equiv 0$ if $p>j$ and $k \leq N_{j+1}$. Hence we may define $g=\sum_{1 \leq j \leq \infty} g_{j}$, which will have the desired properties.

\section{APPENDIX I: DUALITY FOR THE DIFFERENCE QUOTIENT BIALGEBRAS ON THE RIEMANN SPHERE}

We present here the duality result for the difference quotient bialgebras on the Riemann sphere, which is perhaps the simplest case of such duality.

Let $K \subset \mathbb{C}$ be a nonempty compact set and let $G=\mathbb{C} \backslash K$ and $\widetilde{G}=G \cup\{\infty\}=\mathbb{P}^{1}(\mathbb{C}) \backslash K$ be its complements in $\mathbb{C}$ and in the Riemann sphere.

Then $\mathcal{O}(K)$, the algebra of germs of holomorphic functions around $K$ becomes a topological infinitesimal bialgebra when endowed with the comultiplication-derivation given by the difference-quotient $\partial f\left(z_{1}, z_{2}\right)=$ $\left(z_{1}-z_{2}\right)^{-1}\left(f\left(z_{1}\right)-f\left(z_{2}\right)\right)$. Moreover there is a coderivation $L f=(z f)^{\prime}$ with respect to $\partial$, so that $L f-f$ is a derivation of the algebra $\mathcal{O}(K)$.

On the other hand $\mathcal{O}_{\infty}(\widetilde{G})$, the algebra of holomorphic functions $g: \widetilde{G} \rightarrow \mathbb{C}$ which vanish at infinity, $g(\infty)=0$, is also a topological infinitesimal bialgebra with a coderivation. Here, the comultiplicationderivation is given by $-\partial$, the negative of the difference-quotient

$$
-\partial g\left(\zeta_{1}, \zeta_{2}\right)=-\left(\zeta_{1}-\zeta_{2}\right)^{-1}\left(g\left(\zeta_{1}\right)-g\left(\zeta_{2}\right)\right)
$$

(if one or both $\zeta_{1}, \zeta_{2}$ is the point at infinity $\partial g$ vanishes). We also define $\Lambda g=(\zeta g)^{\prime}$ which is a coderivation and $\Lambda$-id is a derivation. 
The well-known duality pairing between $\mathcal{O}(K)$ and $\mathcal{O}_{\infty}(\widetilde{G})$ can be described by

$$
\langle f \mid g\rangle=\frac{1}{2 \pi i} \int_{\gamma} f(z) g(z) d z
$$

where $f$ is defined in an open neighborhood $\omega$ of $K, g \in \mathcal{O}_{\infty}(\widetilde{G})$ and $\gamma$ for each $\omega$ is a finite collection of smooth oriented simple nonintersecting curves in $\omega$, such that the total winding number of $\gamma$ is 0 or 1 around any point outside its support and for the points of $K$ the winding number is 1 (for such $\gamma$ the Cauchy integral formula for points in $K$ holds with integration over $\gamma$, see for instance [2]).

Then we have the kind of duality relations like in Thm. 5.3 of [15].

Fact. If $f, f_{1}, f_{2} \in \mathcal{O}(K)$ and $g, g_{1}, g_{2} \in \mathcal{O}_{\infty}(\widetilde{G})$, then

$$
\begin{aligned}
\left\langle\partial f \mid g_{1} \otimes g_{2}\right\rangle & =\left\langle f \mid g_{1} g_{2}\right\rangle, \\
\left\langle f_{1} f_{2} \mid g\right\rangle & =-\left\langle f_{1} \otimes f_{2} \mid \partial g\right\rangle, \\
\langle L f \mid g\rangle+\langle f \mid \Lambda g\rangle & =\langle f \mid g\rangle .
\end{aligned}
$$

Proof. By density results for holomorphic functions, it suffices to check the first duality relation for the functions $f(z)=(\zeta-z)^{-1}$ for some $\zeta \in \mathbb{C} \backslash K$. After computing $\partial f=\left(\zeta-z_{1}\right)^{-1}\left(\zeta-z_{2}\right)^{-1}$ we can apply three times the Cauchy integral for the outer region of $\gamma$ (containing $\infty)$ to $g_{1}, g_{2}$ and $g_{1} g_{2}$ and show that the left-hand side is $g_{1}(\zeta) g_{2}(\zeta)$ and the right-hand side $\left(g_{1} g_{2}\right)(\zeta)$.

One can deal along similar lines with the second duality relation. One first chooses an open neighborhood $\omega$ of $K$ where $f_{1}$ and $f_{2}$ are defined and a suitable $\gamma$. The second duality relation, written as an equality of integrals over $\gamma$ and $\gamma \times \gamma$, respectively, can be reduced by a density argument to the case when $g(\zeta)=(\zeta-w)^{-1}$ where $w \in \omega$ is a point around which $\gamma$ has winding number 1. After computing $-\partial g=\left(\zeta_{1}-w\right)^{-1}\left(\zeta_{2}-w\right)^{-1}$ we can apply 3 times the Cauchy integral formula and reduce the equality to the obvious one

$$
\left(f_{1} f_{2}\right)(w)=f_{1}(w) f_{2}(w) .
$$

The last duality relation is just that

$$
\begin{aligned}
\langle L f \mid g\rangle+\langle f \mid \Lambda g\rangle & =(2 \pi i)^{-1} \int_{\gamma}\left((z f)^{\prime} g+f(z g)^{\prime}\right) d z \\
& =(2 \pi i)^{-1} \int_{\gamma}\left((z f g)^{\prime}+f g\right) d z
\end{aligned}
$$




$$
=(2 \pi i)^{-1} \int_{\gamma} f g d z .
$$

Remark. In case $K$ is invariant under complex conjugation $z \in K \Rightarrow$ $\bar{z} \in K$, then the same holds for $\widetilde{G}$ and this yields involutions $f^{*}(z)=$ $\overline{f(\bar{z})}$ in $\mathcal{O}(K)$ and $g^{*}(\zeta)=\overline{g(\bar{\zeta})}$ in $\mathcal{O}_{\infty}(\widetilde{G})$. We leave it as an easy exercise to check that with these definitions the bialgebra structures, coderivations and duality are compatible with the involutions along the lines of Thm. 5.3 in [15].

\section{Appendix II: The Fully Matricial $R$-Transform}

It is a rather straightforward fact that the operator-valued $R$-transform [13] is actually part of a fully matricial $R$-transform. We explain this here in the case of a bounded variable, but it is clear that the Grassmannian completion we developed is the appropriate context to deal also with unbounded variables. We also explain how taking the series expansion of the fully matricial $R$-transform one arrives at the unsymmetrized $R$-transform of [5]. In particular the additivity of the unsymmetrized $R$-transform can then be inferred from that of the (symmetric) operator-valued $R$-transform [13].

We shall assume $1 \in B \subset E$ is a unital inclusion of $C^{*}$-algebras and $\Phi: E \rightarrow B$ is a conditional expectation which is a projection of norm 1. Let further $a \in E$ be an element which will be viewed as a $B$-valued noncommutative random variable in $(E, \Phi)$.

Let $G_{a}(b)=\Phi\left((1-b a)^{-1} b\right)$ which is defined for $\|b\|<\|a\|^{-1}$ and which corresponds to $\Phi$ applied to the resolvent of $a$ at $b^{-1}$, when $b$ is invertible. Since $G_{a}(0)=0$ and the differential of $G_{a}$ at 0 is the identity, using the inverse function theorem there is a local inverse $L_{a}$ of $G_{a}$. Since we can make $\left\|\operatorname{id}_{B}-D G_{a}(b)\right\|$ arbitrarily small choosing $\|b\|$ small, it is an exercise for the reader, that there is $\varepsilon>0$, depending only on $C>0$ so that if $\|a\|<C$, then $L_{a}(b)$ is defined for $\{b \in B \mid\|b\|<\varepsilon\}$ and we have $\left\|L_{a}(b)\right\|<(3 C)^{-1}$.

Let $H_{a}(b)=\sum_{k \geq 1}(a b)^{k-1} a$ which is convergent and holomorphic on $\left\{b \in B \mid\|b\|<C^{-1}\right\}$. Then if $\|b\|<C^{-1}$ we have $G_{a}(b)=$ $\Phi(b+b H(a) b)$. If $\|b\|<\varepsilon$, then $\left\|L_{a}(b)\right\|<C^{-1}$ and we have

$$
b=G_{a}\left(L_{a}(b)\right)=L_{a}(b)+L_{a}(b) \Phi\left(H_{a}\left(L_{a}(b)\right)\right) L_{a}(b) .
$$

Then $R_{a}(b)$ which corresponds to " $\left(L_{a}(b)\right)^{-1}-b^{-1}$ " is defined as

$$
R_{a}(b)=\left(1+\Phi\left(H_{a}\left(L_{a}(b)\right)\right) L_{a}(b)\right)^{-1} \Phi\left(H_{a}\left(L_{a}(b)\right)\right)
$$




$$
=\sum_{k \geq 1}(-1)^{k-1}\left(\Phi\left(H_{a}\left(L_{a}(b)\right)\right) L_{a}(b)\right)^{k-1} \Phi\left(H_{a}\left(L_{a}(b)\right)\right) .
$$

Remark that if $\|b\|<\varepsilon$ then $\left\|L_{a}(b)\right\|<(3 C)^{-1}$ and $\left\|H_{a}\left(L_{a}(b)\right)\right\|<C / 2$ which gives $\left\|\Phi\left(H_{a}\left(L_{a}(b)\right)\right) L_{a}(b)\right\|<1 / 6$. This insures that the series for $R_{a}(b)$ is convergent.

The same considerations apply to $a \otimes I_{n} \in \mathfrak{M}_{n}(E)$ as an element of $\left(\mathfrak{M}_{n}(E), \Phi \otimes \mathrm{id}_{\mathfrak{M}_{n}}\right)$. Note that the constants $C$ and $\varepsilon$ stay the same for all $n \in \mathbb{N}$.

Note further that the $G_{a \otimes I_{n}}$ are the components of a stably matricial function on $C^{-1} \mathcal{D}_{0}(B)$. It follows then using 11.5 that the inverse functions $L_{a \otimes I_{n}}$ are the components of a stably matricial function and also the $H_{a \otimes I_{n}}\left(L_{a \otimes I_{n}}\right)$ are the components of a stably matricial function. This then leads to the same conclusion for the $R_{a \otimes I_{n}}$ and moreover this function is also totally bounded. Thus we obtain the following result.

Fact. If $a \in E$ is a noncommutative $B$-valued random variable in $(E, \Phi)$, then there is $\varepsilon>0$, which depends only on $\|a\|$, such that the $R$-transforms $\left(R_{a \otimes I_{n}}\right)_{n \in \mathbb{N}}$ form a $B$-valued totally bounded stably matricial holomorphic function on $\varepsilon \mathcal{D}_{0}(B)$. In particular $\left(R_{a \otimes I_{n}}\right)_{n \in \mathbb{N}}$ has a fully matricial extension to $\varepsilon \widetilde{\mathcal{D}_{0}(B)}$.

Remark. Since $\left(R_{a \otimes I_{n}}\right)_{n \in \mathbb{N}}$ gives rise to a fully matricial function the results about the series expansion apply and the Taylor series has the special form we found in section 13. If $a_{1}, a_{2} \in E$ are $B$-free in $(E, \Phi)$, then $a_{1} \otimes I_{n}, a_{2} \otimes I_{n}$ being $\mathfrak{M}_{n}(B)$-free in $\left(\mathfrak{M}_{n}(E), \mathfrak{M}_{n}(\Phi)\right)=$ $\left(E \otimes \mathfrak{M}_{n}, \Phi \otimes \operatorname{id}_{\mathfrak{M}_{n}}\right)$ we infer [13] that $\left(\mathcal{R}_{\left(a_{1}+a_{2}\right) \otimes I_{n}}\right)_{n \in \mathbb{N}}=\left(\mathcal{R}_{a_{1} \otimes I_{n}}\right)_{n \in \mathbb{N}}+$ $\left(\mathcal{R}_{a_{2} \otimes I_{n}}\right)_{n \in \mathbb{N}}$ is some $\widehat{\alpha \mathcal{D}_{0}(B)}, \alpha>0$. Clearly the same additivity property holds for the Taylor series expansions at the origin. This gives rise to series-versions of the fully matricial $\mathcal{R}$-transform. As noted in [5] the equations defining the operator-valued $R$-transform of [13] and the unsymmetrized $R$-transform formally coincide and hence also formally coincide with the equations defining the fully matricial $R$-transform. Hence we may invoke 13.10 to derive that the series expansion of the fully matricial $R$-transform is the unsymmetrized $R$-transform.

\section{ACKNOWLEDGMENT}

The author's research was supported in part by NSF Grant DMS0501178. The author benefitted from attending and giving lectures at conferences related to the subject of this work, in particular the Free Analysis Workshop at the American Institute of Mathematics in 2006, 
the Takagi Lectures at Tokyo University in 2007 and the free probability workshops in March 2007 at U.C. Berkeley (supported by NSF funds) and at the Fields Institute and BIRS during the academic year 2007-2008. The author also thanks the referee for useful suggestions concerning Appendix II.

\section{REFERENCES}

[1] M. Aguiar, Infinitesimal Hopf algebras, New Trends in Hopf Algebra Theory (La Falda, 1999) Contemp. Math., vol. 267, American Mathematical Society, Rhode Island, 2000, pp. 1-29.

[2] L. V. Ahlfors, Complex Analysis, McGraw-Hill, 1979.

[3] T. Banica and B. Collins, Integration over compact quantum groups, Publ. Res. Inst. Math. Sci. 43 (2007), 277-302.

[4] B. Collins, Moments and cumulants of polynomial random variables on unitary groups, Itzykson-Zuber integral and free probability, International Math. Res. Notices (17):953-982, 2003.

[5] K. J. Dykema, Multilinear function series and transforms in free probability theory, Adv. Math. 208 (2007), 351-407.

[6] J. W. Helton, J. A. Ball, C. R. Johnson and J. N. Palmer, Operator Theory, Analytic Functions, Matrices and Electrical Engineering, CBMS Regional Conference Series in Mathematics, vol. 68, American Mathematical Society, Rhode Island, 1987.

[7] E. Hille and R. Phillips, Functional Analysis and Semigroups, Colloquium Publications, American Mathematical Society, 1982.

[8] S. A. Joni and G.-C. Rota, Coalgebras and bialgebras in combinatorics, Stud. Appl. Math. 61 (1979), no. 2, 93-139.

[9] P. Muhly and B.Solel, Hardy algebras associated with $W^{*}$-correspondences (point evaluation and Schur class functions), Operator theory, system theory and scattering theory: multidimensional generalizations, Operator Theory: Adv. and Appl. 157 (2005), 221-241.

[10] R. Speicher, Combinatorial theory of the free product with amalgamation and operator-valued free probability theory, Mem. AMS 132 (1998), no. 627.

[11] E. M. Stein and G. Weiss, Introduction to Fourier Analysis on Euclidean Spaces, Princeton University Press, Princeton, NJ, 1971.

[12] D. V. Voiculescu, Limit laws for random matrices and free products, Invent. Math. 104 (1991), 201-220.

[13] D. V. Voiculescu, Operations on certain non-commutative operator-valued random variables, Asterisque (1995), no. 232, 243-275.

[14] D. V. Voiculescu, A strengthened asymptotic freeness result for random matrices with applications to free entropy, International Math. Res. Notices (1):4163, 1998.

[15] D. V. Voiculescu, The coalgebra of the free difference quotient and free probability, International Math. Res. Notices 2000 (2000), no. 2, 79-106.

[16] D. V. Voiculescu, Free Analysis Questions I: Duality Transform for the Coalgebra of $\partial_{X: B}$, International Math. Res. Notices 16 (2004), 793-822. 
[17] D. V. Voiculescu, K. Dykema and A. Nica, Free Random Variables, CRM Monograph Series 1, American Mathematical Society, Providence, Rhode Island, 1992.

[18] F. Xu, A random matrix model from two-dimensional Yang-Mills theory, Communications Math. Phys. 190 (2):287-307, 1997. 